\title{
Smart Fluids Using Mechanics: Mechanical Control of Fluid Viscosity Using 3D-printed Functional Particles
}

\author{
Rofiques Salehin
}

Follow this and additional works at: https://researchrepository.wvu.edu/etd

\section{Recommended Citation}

Salehin, Rofiques, "Smart Fluids Using Mechanics: Mechanical Control of Fluid Viscosity Using 3D-printed Functional Particles" (2018). Graduate Theses, Dissertations, and Problem Reports. 7246.

https://researchrepository.wvu.edu/etd/7246

This Thesis is protected by copyright and/or related rights. It has been brought to you by the The Research Repository @ WVU with permission from the rights-holder(s). You are free to use this Thesis in any way that is permitted by the copyright and related rights legislation that applies to your use. For other uses you must obtain permission from the rights-holder(s) directly, unless additional rights are indicated by a Creative Commons license in the record and/ or on the work itself. This Thesis has been accepted for inclusion in WVU Graduate Theses, Dissertations, and Problem Reports collection by an authorized administrator of The Research Repository @ WVU. For more information, please contact researchrepository@mail.wvu.edu. 


\title{
Smart Fluids Using Mechanics: Mechanical Control of Fluid Viscosity Using 3D-printed Functional Particles
}

\author{
Rofiques Salehin
}

Thesis submitted

to the Benjamin M. Statler College of Engineering and Mineral Resources

at West Virginia University

in partial fulfillment of the requirements for the degree of

Master of Science in

Mechanical Engineering

Stefanos Papanikolaou, Ph.D., Chair

Patrick Browning, Ph.D.

Terence Musho, Ph.D.

Department of Mechanical and Aerospace Engineering

Morgantown, West Virginia

2018

Keywords: Molecular Dynamics, Functional Particles, Viscosity, SRD, Jamming

Copyright 2018 Rofiques Salehin 


\title{
Smart Fluids Using Mechanics: Mechanical Control of Fluid Viscosity Using 3D-printed Functional Particles
}

\author{
Rofiques Salehin
}

\begin{abstract}
It is common to manipulate fluid flow properties by infusing additives. Such substances typically include particulate matter that influence fluid mechanical properties. While molecular chemistry can lead to such additives, it has become also possible to explicitly manufacture them using $3 \mathrm{D}$ printing. In this way mechanical properties of fluids may be controlled by infusing variable-shaped functional particles. Here, a way is presented that serves the purpose of controlling viscosity of a fluid by infusing star shaped functional particles. These functional particles are 3D printed using a star shape with variable number of legs and leg lengths. Explicit molecular dynamics simulations of the fluid with infused particles are performed for a variety of structural parameters. The dimensions are chosen with the condition of being easily manufactured. The relation and change of viscosity with respect to the size, shape and packing fraction (in a control volume) is analyzed. In connection to the viscosity's behavior, the structure, diffusion, variation of stress and pressure are also discussed.
\end{abstract}




\section{Acknowledgements}

I want to thank my Research Advisor Dr. Stefanos Papanikolaou for giving me the opportunity to work under his supervision on this super cool project. I am honored to be given the role as a Graduate Research Assistant in his Statistical Mechanics Lab with tremendous research equipment along with other laboratory facilities. I am grateful to Dr. Papanikolaou for his guidance and support that helps me learning so many things that I believe would be an asset for my lifetime.

I would also like to thank my friends and colleagues for supporting me through this last two years that made my abroad life a lot easier. Special thanks to Dr. Rongguang Xu whose selfless suggestions helps me to overcome the obstacles that I could not pass by myself.

Finally, I want to convey my love to my Parents and my Grandma. I could never come to this point without their blessing and unconditional love. 


\section{Table of Contents}

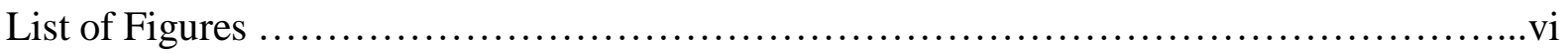

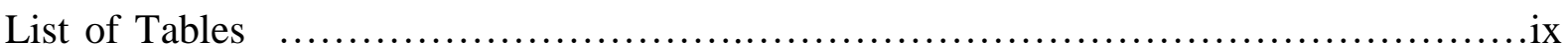

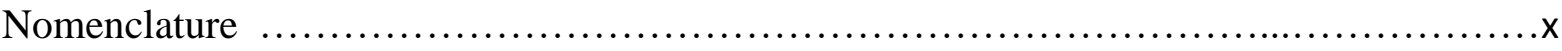

Chapter 1: Introduction \& Thesis Overview.........................................

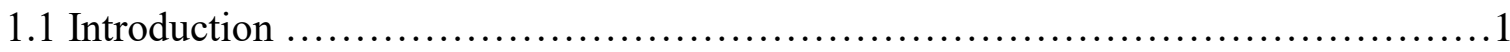

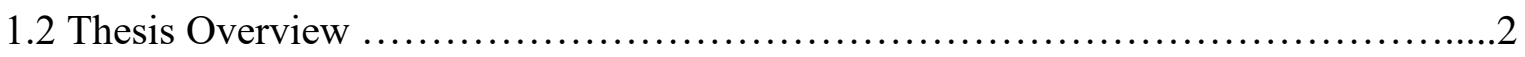

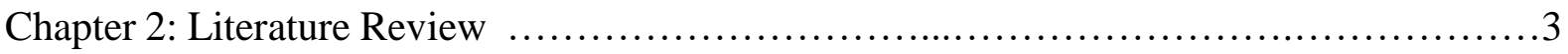

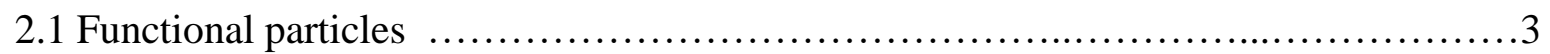

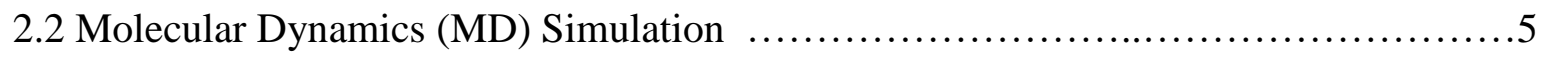

2.3 Jamming in Complex Fluids .................................................... 14

Chapter 3: Methodology .................................................... 17

3.1 MD simulation Model .................................................. 17

3.2 Star particle Generation ................................................... 19

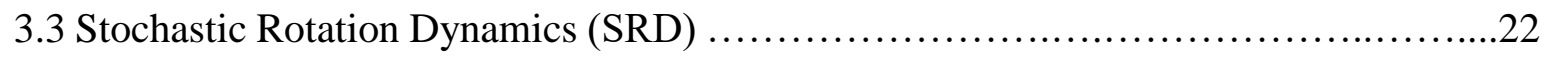

3.4 Observables: Viscosity, Diffusivity, Radial Distribution Function .................25

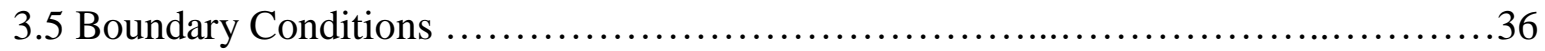

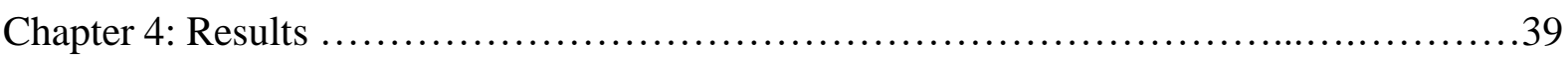

4.1 Shear Stress and Viscosity vs Strain Rate ....................................... 39

4.2 Pressure and Jamming ........................................................... 44

4.3 Jammed System Under Uniform Shear Strain Rate.............................46

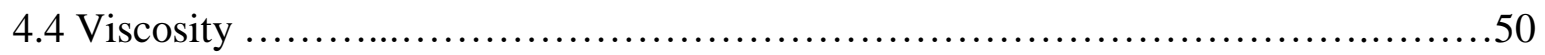

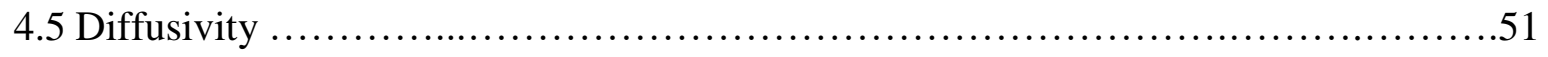

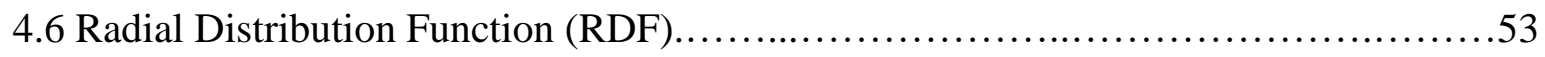

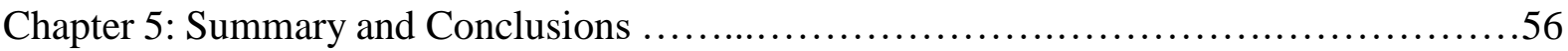


Chapter 6: References 


\section{List of Figures}

Figure 2.1: Stiffest and Softest particle shapes for constituent number of spheres $n=1,2,3,4$ and 5 [34]

Figure 2.2: Periodic boundary condition that has series of images all around identical to the central image [46].

Figure 2.3: The different approaches to computing interactions: (a) all pairs (b) cell subdivision and (c) neighbor list [26]....

Figure 2.4: Jamming phase diagram [16].

Figure 3.1: Single (a) 3leg (b) 5leg and (c) 7leg Star particle with core radius $0.65 \sigma$ and leglength $2.5 \sigma$.

Figure 3.2: Randomly dispersed configuration of 30 Star particles in each system having a square shaped $\left(30 * 30 \sigma^{2}\right)$ simulation box for (a) 3leg (b) 5leg and (c) 7leg.................21

Figure 3.3: 5 leg star particles by (a) 3D printing and (b) LASER cutting. .22

Figure 3.4: (a) Shear stress as a function of shear rate for different Newtonian, Non-Newtonian shear thinning and shear thickening, Bingham plastic and Bingham Pseudoplastic fluid (b) shear stress on a fluid in between two boundary plate where one is moving and another is stationary (c) Viscosity of Newtonian, Shear thinning and shear thickening fluids as a function of shear rate (d) velocity profile for Newtonian, shear thinning and shear thickening fluid[65].

Figure 3.5: Nonequilibrium simulation geometry. Velocity gradient in $v_{x}$ is set up in the $\mathrm{z}$ direction by shearing the liquid. And $\mathrm{x}$ momentum flows towards $\mathrm{z}$ direction that rise momentum flux $j_{z}\left(p_{x}\right)$ through the xy plane [72].

Figure 3.6: Calculation of radial distribution function $\mathrm{g}(\mathrm{r})$. Red particle is the reference particle and blue particles falls within the range of radial increment $\Delta \mathrm{r}$ lined in black[78]...........34

Figure 3.7: Radial distribution function for the Lennard-Jones fluid model [79].............36

Figure 3.8: A square shaped object under (a) simple shear (b) pure shear and (c) simultaneous simple and pure shear [83]. .38 
Figure 4.1: Shear stress vs shear strain rate plotting for (a)3 leg (b)5 leg and (c)7 leg star particles

Figure 4.2: Fitting in Herschel-bulkley model for for (a) 3 leg (b) 5 leg and (c) 7 leg star particles

Figure 4.3: Exponent and yield stress plotting with respect to packing fraction for (a) 3 leg (b) 5 leg and (c) 7 leg star particles.

Figure 4.4: Viscosity plotting with respect to strain rate of (a) 3 leg (b) 5 leg and (c) 7 leg star particles for different packing fraction.

Figure 4.5: Total pressure plotting with respect to packing fraction for (a) 3 leg (b) 5 leg and (c) 7 leg star particles for strain rate 0.001, 0.002, 0.003, 0.004, 0.005, 0.006, 0.007, 0.008, $0.009,0.01,0.02,0.03,0.04,0.05,0.06,0.07,0.08,0.09$ .45

Figure 4.6: Error bar plotting of exponent and jamming packing fraction with respect to strain rate for (a) 3 leg (b) 5 leg and (c) 7 leg star particles. .46

Figure 4.7: Total Pressure vs strain plot for low strain rate (a) 0.001 and high strain rate (b) 0.009 of 3 leg star particles for packing fraction $0.18,0.23,0.28,0.34,0.44,0.49,0.54,0.59$, $0.69,0.74,0.79,0.84,0.94$. Average shear stress with respect to strain is also plotted for strain rate (c) 0.001 and (d) 0.009 of 3 leg star particles for same packing density....

Figure 4.8: Total Pressure vs strain plot for low strain rate (a) 0.001 and high strain rate (b) 0.009 of 5 leg star particles for packing fraction $0.22,0.30,0.40,0.47,0.62,0.72,0.80$. Average shear stress with respect to strain is also plotted for strain rate (c) 0.001 and (d) 0.009 of 5 leg star particles for same packing density....

Figure 4.9: Total Pressure vs strain plot for low strain rate (a) 0.001 and high strain rate (b) 0.009 of 7 leg star particles for packing fraction $0.25,0.37,0.50,0.60,0.84,0.96$. Average shear stress with respect to strain is also plotted for strain rate (c) 0.001 and (d) 0.009 of 7 leg star particles for same packing density....

Figure 4.10: Viscosity vs (a) Leg length and (b) packing fraction plotting for 3 leg, 5 leg and 7 leg star particles .51

Figure 4.11: Diffusivity vs Leg length plotting of small SRD particles and big star particles for (a) 3 leg (b) 5 leg and (c) 7 leg star particles .52 
Figure 4.12: Radial distribution function plot with respect to gradual increment of leg length for (a)-(m) 3 leg star particles with packing fraction $0.18,0.23,0.28,0.34,0.44,0.49,0.54$, $0.59,0.69,0.74,0.79$ and $0.84,(\mathrm{n})$-(t) $5 \mathrm{leg}$ star particles with packing fraction $0.22,0.30,0.40$, $0.47,0.62,0.72$ and $0.80(\mathrm{u})-(\mathrm{z}) 7$ leg star particles with packing fraction $0.25,0.37,0.50,0.60$,

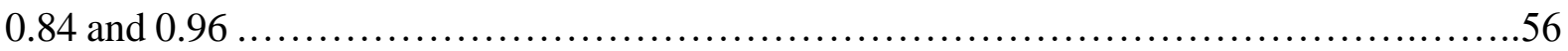




\section{List of Tables}

Table 2.1: Reduced unit of different quantities.....................................

Table 2.2: Simplified schematic of the molecular dynamics algorithm [42] .................14

Table 3.1: Flow models for describing shear stress vs strain rate data [74] .................. 37 


\section{Nomenclature}

\begin{tabular}{|c|c|}
\hline $\mathrm{L}$ & Leg length \\
\hline Nleg & Number of leg in star particle \\
\hline $\mathrm{R}$ & Core radius of star particles \\
\hline $\mathrm{r}$ & Radius of star particles width \\
\hline $\mathrm{n}$ & Number of atoms in each leg of star particle \\
\hline$\varphi$ & Packing fraction \\
\hline $\mathrm{D}$ & Diffusivity \\
\hline $\mathrm{g}(\mathrm{r})$ & Radial distribution function \\
\hline$\theta$ & Angle between two legs in star particle \\
\hline$\eta, \mu^{s}$ & Shear viscosity \\
\hline$\lambda$ & Mean free path \\
\hline$\varphi$ & Packing fraction \\
\hline$\varphi_{c}$ & Jamming packing fraction \\
\hline$\alpha, \beta$ & Exponent \\
\hline$\vartheta$ & Kinematic viscosity \\
\hline$\rho$ & Density \\
\hline$k_{B}$ & Boltzmann Constant \\
\hline$m_{S R D}$ & Mass of SRD atom \\
\hline$r_{c}$ & Cutoff distance \\
\hline$r_{d}$ & Radial distance from core of star particles \\
\hline$L_{0}$ & Simulation box length \\
\hline$\epsilon_{i j}$ & Viscous stress tensor \\
\hline$\sigma_{i j}$ & Total Stress tensor \\
\hline$\delta_{i j}$ & Unit tensor \\
\hline$e_{i j}$ & Strain tensor \\
\hline$E_{i j}$ & Strain rate tensor \\
\hline$\mu_{i j k l}$ & Viscosity tensor \\
\hline$\varepsilon^{v}$ & Viscous shear stress \\
\hline$\mu^{v}$ & Bulk viscosity \\
\hline
\end{tabular}


$\sigma_{y}$ $\tau$

$\dot{\gamma}$

P

$\mathrm{p}$

$j_{z}\left(p_{x}\right)$

$\frac{\partial v_{x}}{\partial z}, \frac{\partial u}{\partial y}$

$\alpha, \beta, \gamma$

$\mu$

L

$\mathrm{H}$

$\mathrm{W}(\mathrm{s})$

c

A

$\mathrm{T}$

$\mathrm{N}$

$\mathrm{dt}, \Delta t$

$\omega(\xi)$

$\xi$

$v_{s}$

$G_{a v}$

M

K

U
Standard deviation ; distance unit in LJ

Yield stress

Shear stress

Strain rate

Hydrostatic Pressure

Momentum

Momentum flux

Velocity Gradient

Angle parameter

Dynamic Viscosity

Lagrangian Function

Hamiltonian Function

Probability of chosen state

Concentration

Prefactor ; slope ; Area

Temperature

Number of time step ; number of particles

Time step

Random rotation matrix

Number of bins

Settling velocity

Average of the Desired quantity

Number of measurements

Kinetic energy

Potential Energy 


\section{CHAPTER-1 \\ INTRODUCTION \& THESIS OVERVIEW}

\subsection{Introduction}

If we turn on the bathroom's faucet then the valve inside the faucet will effectively control the water's apparent viscosity[1]. By this way, we only have control at faucet's valve. Although it works well in our household, but implementing the same hydraulic valve phenomena in every case would be cumbersome or sometimes impossible. A characteristic example is the case of vehicle suspensions which should adapt to road conditions as they may be monitored through sensors in the vehicle. We are interested in finding the possibility of continuous control of damping characteristics of the shock absorber fluid in the suspension. There are plenty of analogous examples exist where valves are inefficient. Semi-active prosthetic limb, cockpit seats in the helicopter aviation industry, seat damping in construction vehicles and earthquakeresistant damping in the building are few of such examples. In the past, there have been suggestions of functional dampers [2]-[4], such as magnetorheological (MR) fluids [5]-[9] , Electrorheological (ER) fluids [10]-[12], Ferrofluids [13] etc. However, these fluids are heavily limited by their ultra-low shear-yield stresses $(100 \mathrm{kPa})$, which have prohibited commercial applications. Moreover an external field is required to successfully implement this concept. Therefore, we aimed to develop a novel route towards engineering functional dampers using 3D-printing of generically applicable functional particles [14] made of light and strong polymeric and metallic materials.

We implement the way to control damping characteristics of generic fluids by infusing functional particles. The idea is based on the engineering use of the concept of collective jamming [15]-[21] that takes place in randomly packed ensembles, such as sand piles and other particular matter. Jamming takes place when the packing fraction of the constituent grains in a finite volume, is large enough that the system develops elastic properties and finite yield stress. For example, think of filling up a metallic box with M\&Ms: if M\&Ms are packed efficiently, it is not possible to compress them further, which implies the emergence of solid elasticity. The phenomena of jamming and fluid-to-jammed transition are general and are characterized by 
various aspects that may become useful in various fields of our society, from human traffic jams to cell migration in our body.

\subsection{Thesis Overview}

In this study we have simulated a complex fluid system under shear. We have infused functional particles in generic fluid similar to smart fluid mechanics [22], [23] without any external magnetic or electric sources. The infused functional particles comes to jammed state under the applied shear. Due to this jamming transition fluid's viscosity rises with respect to the systems packing density. Our purpose is to observe and visualize the variation of the fluid's viscosity as a function of packing fraction. The viscosity calculation is related to the applied shear strain rate and resulting shear stress. Therefore, we emphasized mainly on the stress calculation and its variation with strain rate. The diffusivity and pair correlation function is also studied afterwards to understand the system concentration. We have used Molecular Dynamics (MD) simulation [24]-[28] for both macroscopic and microscopic length scale in a popular MD simulation package named Large-scale Atomic/Molecular Massively Parallel Simulator (LAMMPS) [29]-[31] written in C++. We have used reduced (LJ) unit system in our simulation. The simulation results could be transformed into conventional units and compare with the experimental results.

This paper has reviewed few of the works on functional particles and jamming transition in chapter 2. Here we also discussed the MD simulation method briefly. The methodology section in chapter 3 includes the details of our simulation model and how we generated our functional particles. It also has detailed discussion about the stochastic rotation dynamics (SRD). We have used this dynamics to imbue the fluidic property from numerous ideal point particles that interacts with specific pair potential. The observable properties along with respective boundary conditions are also added in this chapter. In chapter 4 we represent all the results by processing the data found from the simulations. We have discussed and analyzed our findings from the plottings on viscosity, diffusivity, radial distribution function, pressure, shear stress and strain rate. Finally chapter 5 summarizes and concludes our findings and recommendations for future work. 


\section{CHAPTER-2 \\ LITERATURE REVIEW}

\subsection{Functional particles}

The functional particles are functionalized in different ways to get desired properties or modify the current properties. These properties could include surface modification, biocompatibility, electronic device fabrication, drug delivery, water purification, coating application, imaging agents etc. But we will keep our focus on functionalizing the shape and surface of the particle for desired response. Because this functionalization of particle could influence the fluid mechanical properties such as viscosity [32], [33], adhesion between infused particles with the generic fluid.

Marc Z. Miskin and Heinrich M. Jaeger proposed through evolutionary computing that the mechanical response such as modulus of elasticity of a particle is mostly depends on the shape of granular material because this shape determines both the packing arrangement and contact interaction [34]. This dependency is demonstrated by computer simulation using artificial evolution algorithm [35], [36]. The shape could be created in any form by joining granular materials. Because of these granular particles the surface asperity is generated automatically. Also tri-axial compression test proves this dependency.

Functional particle consists with two sphere shaped granular particle would give much stiffer and stronger packing [37] than a single sphere. It would be clearly understood from stressstrain curve for single and double spheres. The more compact the shape the more stiff the packing that cause the rise of elastic modulus $E$. While measuring these responses the initial condition, boundary condition and material property is also considered. But if the number of spheres is more than two then the opening angle at the central sphere plays the role for particle shape. The shape for larger number of sphere is an inexhaustible parameter. To find the 
particular shape for desired behavior efficiently from the numerous potential configurations of particles the Evolutionary Computing is used. The main idea of this computing is to start with few different shapes of particles and measure the performance of each shape. Then choose the best of these and improve that specific one by modifying the shape into few more closely related shapes. And following this same iteration creates finally creates the best offspring that gives the best result. By this approach they found the stiffest and softest response that gives the largest and smallest elastic modulus respectively. In general for different number of spheres the softest response is found for rod like (linear) shapes. For three, four and five spheres most compact shapes are found in the form of triangular, rhombic and triangular bi pyramid shapes respectively. This response are stiffest at high packing because the particles could not slide past each other and thus increases the local contact that raise the stress. On the other hand the linear ones easily slide past each other. Near Jamming point the strain stiffening also depends on particle shape. The same algorithm could found the best shape for strain stiffening by determining the second derivative of stress with respect to strain.

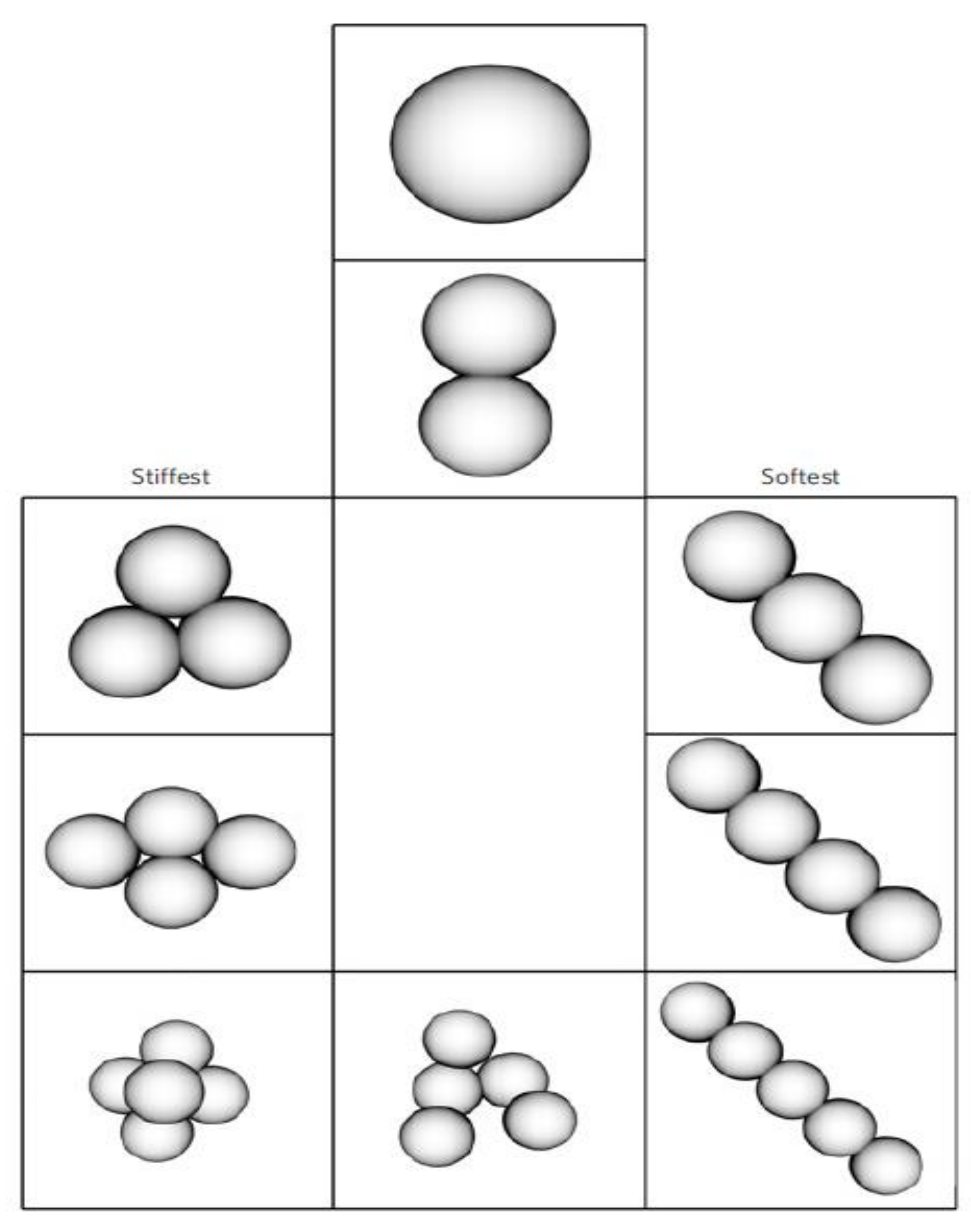

Figure 2.1: Stiffest and Softest particle shapes for constituent number of spheres $n=1,2,3,4$ and 5 [34] 
The mechanical properties such as adhesion, flowability depends largely on shape and surface asperity of the particles. The adhesion on iron plate due to asperity is studied by Helmar Krupp [38]. Likewise, Leopoldo Massimilla and Giorgio Donsi examines the adhesion on silica for surface asperity [39]. But Akinobu Otsuka, Kotaro Iida, Kazumi Danjo and Hisakazu Sunada did the experimentation to find out the best shape for adhesion property using impact separation method using 26 different powdered materials [40].

The surface of power materials are functionalized by removing protuberances and fine particles by dissolving into different types of acid solution for specific materials such as silica sand into hydrofluoric acid, sulfadimethoxine into dilute ethanol and calcium carbonate into dilute hydrochloric acid.

The impact separation method determines the adhesion force between particles placed on glass in a measuring cell attached with motor driven hammer. In room temperature this experiment is conducted for all different types of particles and percentage of particles remaining after the shock is plotted against separation force to compare the adhesion force for each material. The result founds that the spherical shaped particles regardless the materialistic property shows higher adhesive forces. When the surfaces are functionalized by removing the protuberances this adhesive force is increased about ten-times. If the concentration of acid is increased while dissolving the particles to functionalize then the desired adhesive force is increased further. Moreover,adhesive force increases initially for the increase of treatment time but for sufficiently longer treatment time it tends to come at a constant value.

\subsection{Molecular Dynamics (MD) simulation}

Molecular Dynamics (MD) simulation is a typical simulation technique based on statistical mechanics for systems with many particles. MD simulation runs based on Newton's second law of motion and requires complete information about position and momentum of each particles inside the system. MD produces the average values for series of measurements of a particular system like statistical mechanics [41] does. This simulation technique could be categorized into many forms by considering whether the interactions are short ranged or long ranged ; the system is open, closed or isolated ; the ensemble that is used are micro canonical, canonical or isothermal-isobaric ; the interaction potential functions are simple or by step ; 
interactions involved pair particles or multi-particle; the constituent particles are atoms or molecules and if molecule whether it is rigid or flexible and so on. MD simulation is most frequently used in liquids. The reason is both solid and gas has better theoretical foundation than liquid. Solids has fixed lattice position and gases has weak interaction between its atoms but liquids there is interaction but no ordered structure.

MD simulation does not depend on the initial state of the system. For same system with different initial condition should not affect the results. But to reach in an equilibration state where there would be no memory of initial state could take place with different speed. Therefore adequate time should be provided. Initial velocities are set in random directions depending on temperature.

MD could have couple of unit style such as $1 \mathrm{j}$, real, metal, si, cgs, electron, micro or nano. Among these units lj unit style is dimensionless and therefore known as reduced unit [25]. This reduced dimensionless unit system is expressed in terms of distance $\sigma$, mass m, energy $\varepsilon$ and Boltzmann constant $k_{B}$. There are several benefits of using reduced unit. For MD simulation it is better to use values that is not much far away from unity as there are quite a few of them whose values are much smaller than 1 in atomic scale. Also the equations of motion are simplified by using this unit system. Finally the most important feature of reduced unit is scaling. Once the properties of a system is measured in reduced unit then it could convert to any unit system for different systems. That would save much of computational work and effort. We are attaching a table consisting the units of several parameters in LJ unit.

\begin{tabular}{|c|c|}
\hline Property & LJ unit \\
\hline Mass & $\mathrm{m}$ \\
\hline Distance & $\sigma$ \\
\hline Time & $\tau=\left(m \sigma^{2} / \varepsilon\right)^{\frac{1}{2}}$ \\
\hline Energy & $\varepsilon$ \\
\hline Velocity & $\sigma / \tau$ \\
\hline Force & $\varepsilon / \sigma$ \\
\hline Torque & $\varepsilon$ \\
\hline Temperature & $\varepsilon / k_{B}$ \\
\hline
\end{tabular}




\begin{tabular}{|c|c|}
\hline Pressure & $\varepsilon / \sigma^{3}$ \\
\hline Dynamic Viscosity & $\varepsilon \tau / \sigma^{3}$ \\
\hline Diffusivity & $\sigma^{2} / \tau$ \\
\hline Stress & $\varepsilon / \sigma^{3}$ \\
\hline density & $m / \sigma^{3}$ \\
\hline
\end{tabular}

Table 2.1: Reduced unit of different quantities.

The microscopic state of a system is formed by considering all the particles position and momentum. For coordinate $(\mathbf{q})$ and momentum (p) of each particle $\mathrm{i}$ we could write the Hamiltonian form of the equation of motion as the sum of kinetic energy as a function of momentum and potential energy as a function of position [24].

$$
\begin{gathered}
q=\left(q_{1}, q_{2}, q_{3}, \ldots \ldots q_{N}\right) \\
p=\left(p_{1}, p_{2}, p_{3}, \ldots \ldots p_{N}\right) \\
H(q, p)=K(p)+U(q)
\end{gathered}
$$

For molecular mass $m_{i}$ and index $\alpha$ that indicates the component of momentum $\mathrm{p}$ in $\mathrm{x}, \mathrm{y}, \mathrm{z}$ directions the kinetic energy $\mathrm{k}$ is expressed as,

$$
K=\sum_{i=1}^{N} \sum_{\alpha} p_{i \alpha}^{2} / 2 m_{i}
$$

And the potential energy $U$ that contains the intermolecular interaction information is in general expressed as following,

$$
U=\sum_{i} v_{1}\left(r_{i}\right)+\sum_{i} \sum_{j>i} v_{2}\left(r_{i}, r_{j}\right)+\sum_{i} \sum_{j>i} \sum_{k>j>i} v_{3}\left(r_{i}, r_{j}, r_{k}\right)
$$

Where the first term $v_{1}$ represents the external effect on the system. The second term $v_{2}$ is the pair potential that depends only on the pair separation distance $r_{i j}=\left|r_{i}-r_{j}\right|$. The third term represents the triplets. The summations considers only the distinct pairs $(i, j)$ or triplets $(i, j, k)$ once (i.e. either ij or ji for pairs).

The equations of motion could be written in different forms [28]. One of the fundamental form is Lagrangian equation of motion written as [24], 


$$
\frac{d}{d t}\left(\delta L / \delta \dot{q}_{k}\right)-\left(\delta L / \delta q_{k}\right)=0
$$

Where Lagrangian function L dependent upon $\mathrm{q}$ and $\dot{q}$ is defined with respect to Kinetic energy $\mathrm{K}$ and potential energy $\mathrm{U}$ as following,

$$
\mathrm{L}=\mathrm{K}-\mathrm{U}
$$

In Cartesian coordinates $r_{i}$ the Lagrangian equation becomes,

$$
m_{i} \ddot{r}_{i}=f_{i}
$$

Where $m_{i}$ is the mass of atom $\mathrm{i}$ and $f_{i}$ is the force on that atom defined as,

$$
f_{i}=\nabla_{r_{i}} L=-\nabla_{r_{i}} U
$$

Now the momentum equation will be,

$$
p_{k}=\delta L / \delta \dot{q}_{k}
$$

If there is molecules instead of atoms then the same equation could be applied for the center of mass of the molecule i .

Now for Hamiltonian form of equation of motion could be written as,

$$
H(p, q)=\sum_{k} \dot{q}_{k} p_{k}-L(q, \dot{q})
$$

Where $\mathrm{H}$ is the Hamiltonian function that gives the momenta feature as following,

$$
\begin{gathered}
\dot{q}_{k}=\delta H / \delta p_{k} \\
\dot{p}_{k}=-\left(\delta H / \delta q_{k}\right)
\end{gathered}
$$

For Cartesian coordinates the Hamiltonian equation becomes,

$$
\begin{gathered}
\dot{r}_{i}=p_{i} / m_{i} \\
\dot{p}_{i}=-\nabla_{r_{i}} U=f_{i}
\end{gathered}
$$

The equations of motions are solved at each time step using simulation algorithm that is fast, requires less memory, permit the use of shorter as well as longer time step, could duplicate trajectory as closely as possible, satisfy conservation laws for momentum and energy and 
simple form with easy programming. Most widely used algorithm for time integration is the Verlet algorithm [42] adopted by Verlet and Stormer. This method gives the direct solution of $2^{\text {nd }}$ order equation based on the current position $r(t)$, position at previous time step $r(t-\delta t)$ and acceleration a $(\mathrm{t})$. The position is calculated as follows with an error of order $\delta t^{4}$ [24],

$$
r(t+\delta \mathrm{t})=2 r(t)-r(\mathrm{t}-\delta \mathrm{t})+\delta t^{2} a(t)
$$

The velocities are useful for estimating kinetic energy and therefore calculated as following with an error of order $\delta t^{2}$,

$$
v(t)=\frac{r(t+\delta \mathrm{t})-r(t-\delta \mathrm{t})}{2 \delta \mathrm{t}}
$$

$\mathrm{v}(\mathrm{t})$ can only be calculated when $r(t+\delta \mathrm{t})$ is known. Also verlet algorithm is properly centered between $\mathrm{r}(t+\delta \mathrm{t})$ and $\mathrm{r}(t-\delta \mathrm{t})$ makes the time reversible. Finally, the position advances in single stage rather than two stage as predictor-correction model. For this reasons velocity calculation has few deficiencies. This deficiencies could be overcome by leap-frog method that use the following algorithm [43],

$$
\begin{gathered}
r(t+\delta \mathrm{t})=r(t)+\delta \mathrm{tv}\left(\mathrm{t}+\frac{1}{2} \delta \mathrm{t}\right) \\
v\left(t+\frac{1}{2} \delta \mathrm{t}\right)=v\left(t-\frac{1}{2} \delta \mathrm{t}\right)+\delta \mathrm{ta}(\mathrm{t})
\end{gathered}
$$

Current position $\mathrm{r}(\mathrm{t})$ and acceleration $\mathrm{a}(\mathrm{t})$ is stored along with mid time step velocity $\mathrm{v}(\mathrm{t}-$ $\left.\frac{1}{2} \delta \mathrm{t}\right)$. The next mid timestep velocity $\mathrm{v}\left(t+\frac{1}{2} \delta \mathrm{t}\right)$ is found from the implementing the velocity equation. By this way current velocities are calculated as,

$$
v(t)=\frac{v\left(t+\frac{1}{2} \delta \mathrm{t}\right)+v\left(t-\frac{1}{2} \delta \mathrm{t}\right)}{2}
$$

The advantage is by scaling the velocity the simulation energy is achieved. But still it does not handle the velocity satisfactorily. Therefore Velocity Verlet algorithm is proposed by Swope, Andersen, Berens and Wilson [44] which stores position $\mathrm{r}(\mathrm{t})$, velocity $\mathrm{v}(\mathrm{t})$ and acceleration $\mathrm{a}(\mathrm{t})$ at time $\mathrm{t}$ with minimum round off-error as the following ,

$$
\begin{aligned}
& \left.r(t+\delta \mathrm{t})=r(t)+\delta \mathrm{tv}(\mathrm{t})+\frac{1}{2} \delta t^{2} \mathrm{a}(\mathrm{t})\right) \\
& v(t+\delta \mathrm{t})=v(t)+\frac{1}{2} \partial t[a(t)+a(t+\partial t)]
\end{aligned}
$$


The Verlet algorithm could be recovered from this by removing the velocities. It does involves two stages. First the new position $\mathrm{r}(t+\delta \mathrm{t})$ is calculated and then mid time step velocity is calculated as

$$
v\left(t+\frac{1}{2} \delta \mathrm{t}\right)=v(t)+\frac{1}{2} \partial t a(t)
$$

Then the force and accelerations at that $t+\delta \mathrm{t}$ is calculated which follows by the final velocity calculated as,

$$
v(t+\delta \mathrm{t})=v\left(t+\frac{1}{2} \delta \mathrm{t}\right)+\frac{1}{2} \partial t a(t+\delta \mathrm{t})
$$

In experiment usually temperature and pressure are controlled or fixed to a constant value but in Molecular Dynamics pressure, temperature, energy and volume any of these two could be controlled and fixed based on the requirement. If volume and energy is choose it is called micro canonical (NVE) ensemble average. Here N is the number of atoms. Similarly canonical (NVT) or isothermal-isobaric (NPT) ensemble average fixed the volume and temperate or pressure and temperature respectively. In microscopic level the thermal fluctuation could be large that could make the precise measurement difficult. Therefore modifying dynamics using ensembles makes the MD simulation reach in equilibrium directly. These ensembles could be applied by a feedback mechanism where the controlled parameter fluctuates around the desired mean value or constraint mechanism where controlled parameter is kept constant strictly. There are other ways also to control the desired parameter such as coupling or simply resetting the parameter at each time step [26]. For example, there are several methods such as constraint method, extended system method and stochastic method to keep the temperature constant [45]. In the first method by imposing constraint, total kinetic energy is kept constant. As temperature is related with kinetic energy therefore temperature also remain constant. For non-equilibrium molecular dynamics simulation Gaussian thermostat is used as the constraint. This thermostat imposed a force term in the equation of motion to restrict the particle motion. The extended method system introduced additional degree of freedom that allows the total energy to fluctuate while in thermal contact with heat bath. Finally, the stochastic method implements the concept of random force affecting thermal motion for macroscopic system in microscopic system. A friction force and random force is added to the equation of motion where both of these forces 
are interconnected with temperature. Therefore, when temperature is increased due to random force, friction force is reduced and thus kept the temperature constant.

For MD simulation the smaller the system the lower the computation requires. Therefore the system size needs to be determined appropriately so that it becomes small enough to make the computation less expensive and at the same time big enough to capture the actual behavior of total system. Unless the goal is to find the behavior at the boundary one could eliminate the walls to capture the original interior state. The boundary free from walls could be captured by periodic boundary condition. For this kind of boundary condition atoms leaves from one face of the simulation and enters immediately into the simulation box from the opposite face. During this periodicity wraparound effect also takes into account while updating equations of motion and interaction computations. Wraparound effect could be understood by a 1D model in $\mathrm{x}$ direction extends from $-\mathrm{L} / 2$ to $\mathrm{L} / 2$. If an atom coordinate $\mathrm{x}$ becomes greater than $\mathrm{L} / 2$ then its coordinate is adjusted by subtracting $\mathrm{L}$ from it that makes the final coordinate staying inside the box. Similarly if the $\mathrm{x}$ coordinate is lower than $-\mathrm{L} / 2$ than the coordinate is adjusted by adding $\mathrm{L}$ to its coordinate. In general rectangular shaped and rectangular prism shaped region is easier to handle periodicity for 2D and 3D case respectively. Although other shapes could also be used for periodicity.

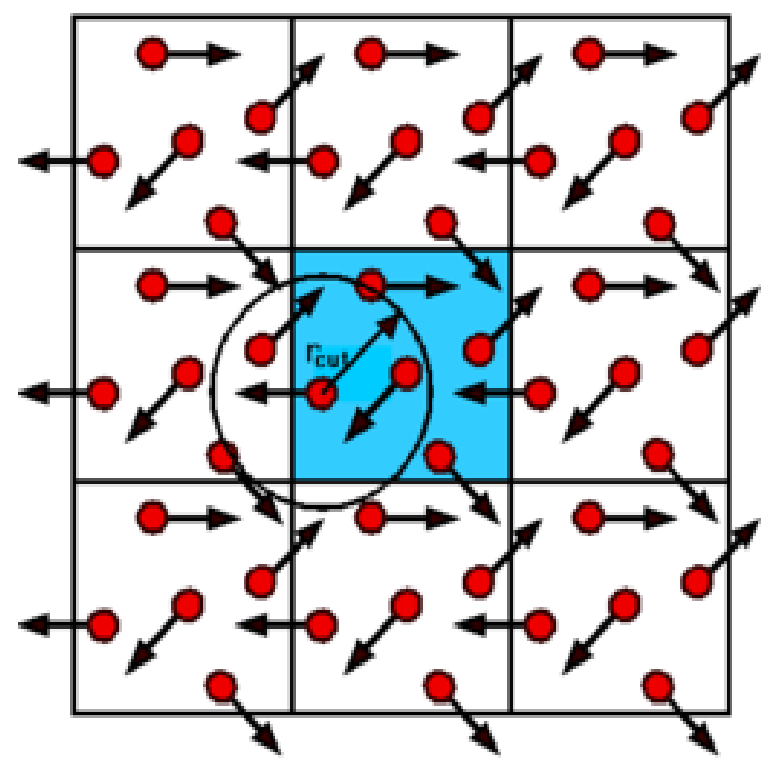

Figure 2.2: Periodic boundary condition that has series of images all around identical to the central image [46]. 
In MD it chooses one particle and considers the interaction with all other particles within a distance of potential cutoff called $r_{c}$ and thus reduces the computational cost by avoiding the interactions outside the cutoff distance. The time required to examine whether the particles are separated by less than the cutoff distance is proportional to $N^{2}$ where $N$ is the number of particles. This list of neighbors are updated after each time steps. Between this updates MD does not check all particles rather only the particles that falls into the neighbor list. To speed up the neighbor list calculation there are couple of ways such as cell subdivision, verlet neighbor list etc.

Cell subdivision is the method of organizing the information of atoms position to reduce the computational effort by dividing the simulation region into lattices of small cells with the edge length equals to the cutoff distance $r_{c}$. Atoms in one cell could only interact with the atoms inside that cell or the adjacent cells only. By this way it has to consider 14 neighboring cells for $3 \mathrm{D}$ or 5 neighboring cells for $2 \mathrm{D}$ case. Separate lists are required to gather the information of each cell. But the number of particles could vary rapidly from cell to cell and therefore to avoid the waste of storage linked list approach is used.

Neighbor list method enables the use of a neighbor list for several successive time steps. It replaces the cutoff $r_{c}$ by $r_{n}$ where $r_{n}=r_{c}+\Delta r$ and $\Delta r$ is generally known as skin distance. If the microscopic environment changes slowly then this approach works much better as the neighbors remain valid for typically 10 or 20 time steps even for small skin distance. There is also an approach called multiple-time step method where there are two neighbor list primary and secondary. Primary neighbors includes all the atoms in a distance $r_{p}$ larger than the cutoff distance $r_{c}$ whereas secondary neighbors are the atoms in between the $r_{p}$ and $r_{c}$. 


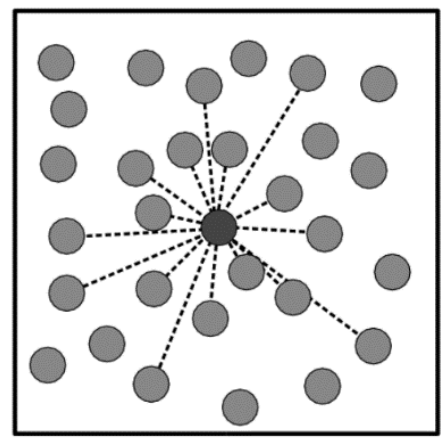

(a)

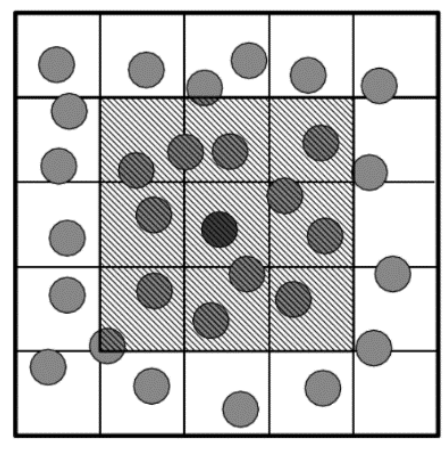

(b)

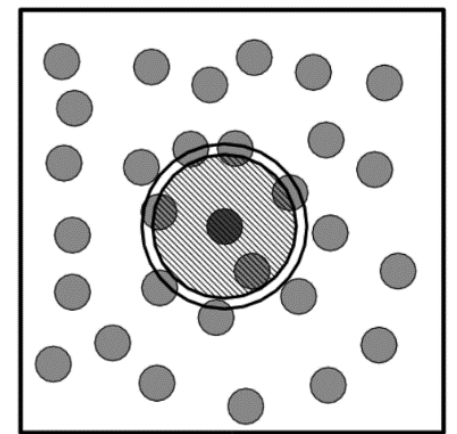

(c)

Figure 2.3: The different approaches to computing interactions: (a) all pairs (b) cell subdivision and (c) neighbor list [26]

MD is computationally intensive because of many time steps along with subsequent computations in each time step. Therefore to speed up the simulation we could reduce the amount of computation in each time step, reduce the number of time steps and/or the time step size by taking the minimum required run time, run the simulation in parallel running, Use GPUs to speed up the performance of computers [47]. 


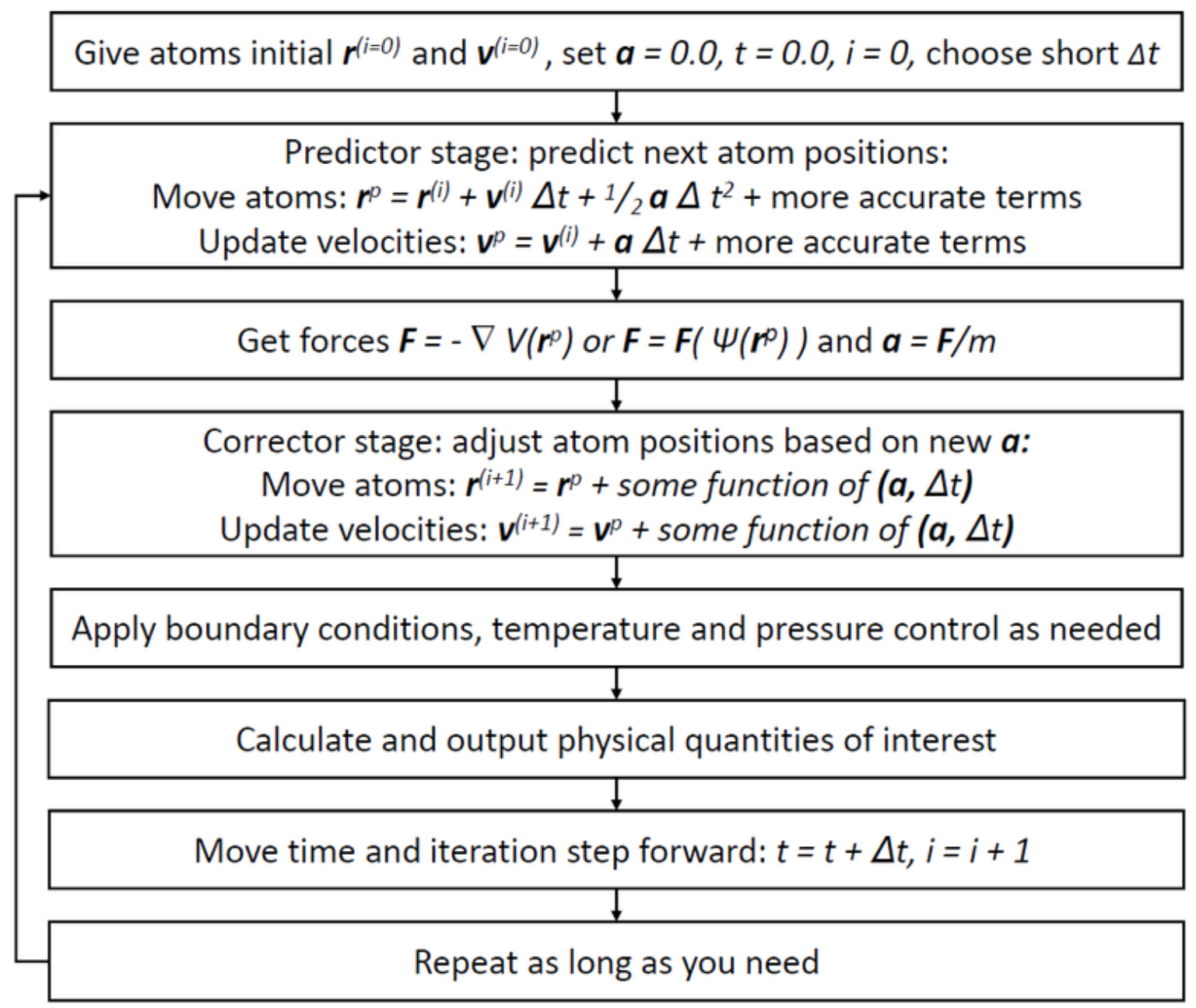

Table 2.2: Simplified schematic of the molecular dynamics algorithm.[48]

\subsection{Jamming in Complex Fluids}

Jamming is a very common phenomena in our everyday life. Traffic jams in the roads and highways, pile of sand, shaving foam, powdered raw materials that clog the conduit; all of them are the result of jamming.

Jamming transition is the way of a system from flowing material or liquid to gain rigidity. But during this transition to rigidity the internal structure remains disordered. Different disordered matter such as foams, colloidal suspensions, granular material, glasses etc. can be jammed and turn into rigid state that deformed elastically due to applied stress. Fluid could also be solidified 
by crystallization but in that case the system is rather ordered. Jamming could also be defined as the physical process that causes the rise of viscosity by varying the particle density of materials in mesoscale such as complex fluids, polymers etc. Jamming causes the phase transition of materials which is highly nonlinear with packing density. It is pretty similar to glass transition but in that case the liquid state turned into solid by cooling but in this case it is done by increasing the density. Jamming transition has both first order and second order transition. Jamming transition could be controlled by varying thermodynamic variable (i.e. temperature, density) or mechanical variable (i.e. stress). A liquid turns into glass when temperature is lowered that increases the viscosity. Flowing foam becomes rigid when applied stress is lowered. Colloidal suspension stops flowing when its density is increased. These different control parameter does not actually change the structure but brings the system into rigid state. The phases remain amorphous and order parameter has not been identified to differentiate the phases [49].

Frictionless spheres interact with repulsive force near zero temperature is considered as the simplest model of jamming. Jamming of frictionless deformable particles with repulsive interaction is caused by deformation due to pressure on packing density. When deformation stops the pressure also stops and jamming is also lost that makes the system loss its rigidity. Therefore the system geometry and its rigid behavior is dependent to each other. The average number of contact between the particles are very important for the system to hold its rigidity. If this contact number reaches to a minimal value the system loses its rigidity. A precise value that indicates the minimum contact number between the particles to demonstrate the jamming property is called isostatic contact number [20]. The jamming may vary from one system to another depending on the constituents of that system. Both static and sheared system could lead to jamming. System could be jammed under shear by straining and also the jamming could break by breaking the chain when stress reaches to a required level. Also a system which consumed energy could also be jammed. By increasing and decreasing the packing fraction or applied stress value above and below the yield point could jam and unjam the system. Density, stress and temperature are the three key factor regarding jamming transition. The density is dependent upon many factors such as particle shape, deformability, friction, dispersity etc.

If shear is applied in system consists of colloidal hard particles then over a certain value of shear stress the system could jam because of the forming of force chain among the particles in 
the sheared direction. This makes the material act as a solid because of the rise of contacts in between the particles that creates array of force chains that shows resistance against the applied force to deform. This type of solid does not act like the conventional elastic or plastic solids rather than like a new solid named fragile. Fragile means that it could not support even an infinitesimal small load elastically. Fragility could be distinguished into 2 types. One is bulk fragility that considers body force and the other is boundary fragility that considers forces at the boundary. The responses could also be considered as macro fragile that considers the mean orientation or micro fragile that considers the individual contacts [50].

Cates et al. named the material that cause jamming due to applied stress and results a solid like property as fragile material. Jammed particles are stuck and could not escape from their position in a small confined space. But if stress is applied from different direction the jamming could breaks up. Therefore new theoretical description is proposed for fragile matter. As cates et al. considers non-deformable particles therefore deformation of material due to stress is not useful consideration. It also considers the system supports applied load as jamming force in the same direction but not as an elastic body. It completely ignore the effect of strain. So the new concept of fragile matter includes the particles deformation property. The problem arises with the consideration that there is no attractive force nor any thermal motion. But the attractive force could be replaced with repulsive forces in confined space. This is similar to say that instead of attracting each other due to attractive internal force they are coming closer due to externally applied force imposed by pressure. Changing temperature could change the interaction force between particles. The higher the temperature lower the attractive force and the lower the temperature the higher the attraction force becomes. Therefore by lowering the temperature it is possible to increase the attraction force that would replace the necessity of stress applied externally and results in a jammed state. Liquids could jam the same way by lowering temperature and this happens because of the increase of viscosity below its freezing point. The same way jamming could be unjammed by increasing the temperature. This density could be varied by applied stress and temperature. So there is a definite relation between these parameters which could be easily understood by the Figure 2.4 illustrated below [16]. 


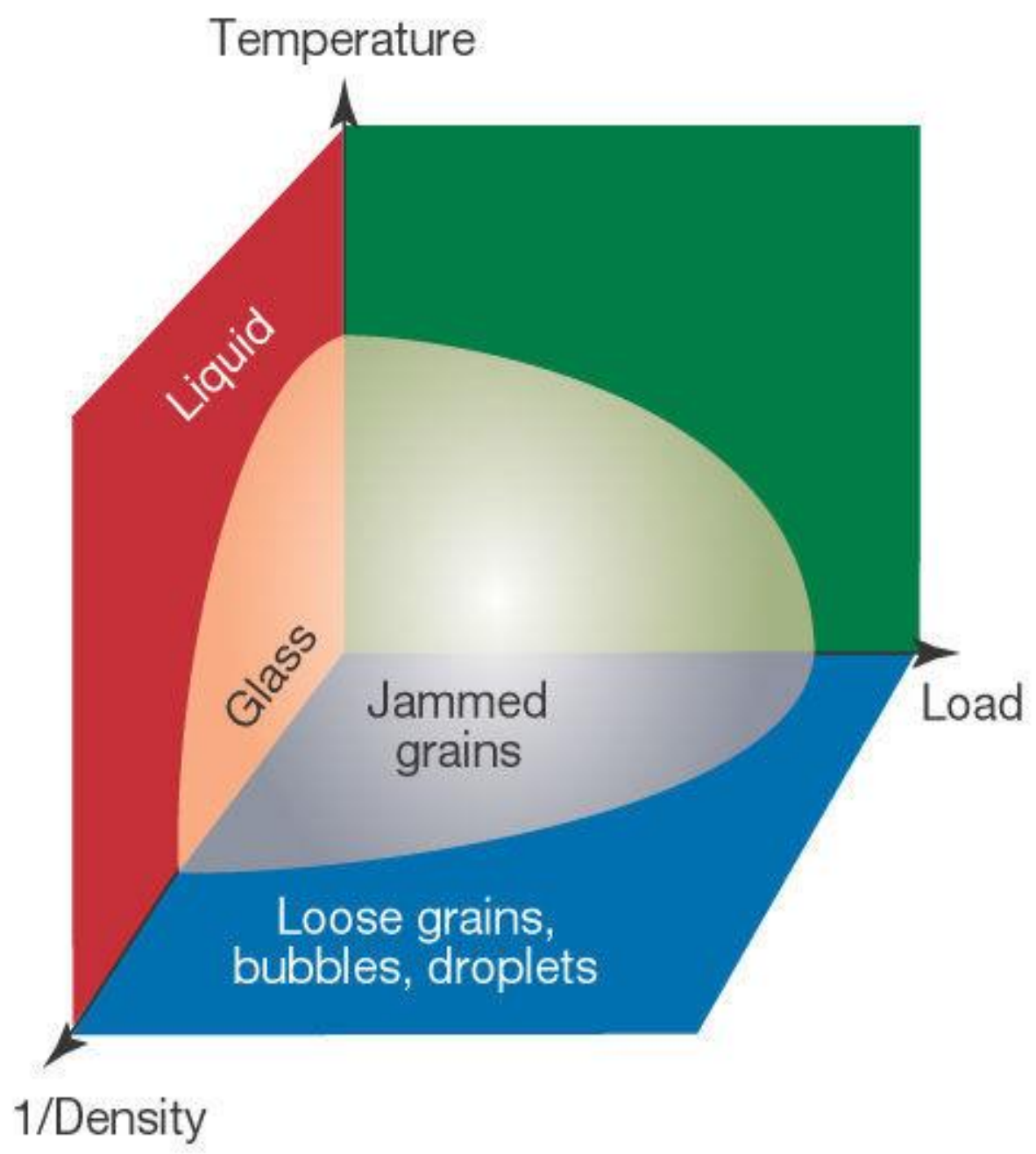

Figure 2.4: Jamming phase diagram [16] .

\section{CHAPTER-3}

\section{METHODOLOGY}

\subsection{MD simulation model}

For running our desired simulation we have chosen a vastly popular MD simulation package LAMMPS. It uses neighbor list method to keep track of surrounding particles. We could run the simulation both is serial and parallel mode quite effectively. 
We have chosen a square shaped (30*30 square unit) two dimensional simulation box having periodicity in both $\mathrm{X}$ and $\mathrm{Y}$ directions. From the available unit system (i.e. lj, real, metal, si, cgs, electron, micro, nano) in LAMMPS we choose the LJ which is also named as reduced unit. The velocities for two different type of particles are created using random number generator at specified temperature. The types are assigned as small for SRD particles that act as fluid and big for functional star particles we infused varying the leg lengths and number of legs by reading the star data file we have created with asperities because of our requirement. We have set the keywords such that each processor independent on the number of processor used creates velocity based on random seed number that is generated based on atoms coordinates.

We have chosen the soft potential for pairwise interaction which is a repulsive potential that is soft at the starting but hardens the interaction over time. It computes pairwise interaction as the following [51],

$$
E=A\left[1+\operatorname{Cos}\left(\frac{\pi r}{r_{c}}\right)\right], \text { where } r_{c}>r
$$

Here, $\mathrm{A}$ is a prefactor which is in energy unit and $r_{c}$ is the cutoff distance. It is clear from the equation that $\mathrm{E}$ does not blows up even if when $r$ goes to 0 and hence it is very effective for pushing apart overlapped atoms. We could ramp the prefactor value from an initial value to our desired value with respect to time.

The neighbor list is updated as a list with a skin distance $(\Delta r)$ equals 0.3 which is a default value for $\mathrm{lj}$ unit in LAMMPS. Also we choose the faster algorithm named bin to build the neighbor list where binning operation scales linearly with $\mathrm{N} / \mathrm{P}$. Here $\mathrm{N}$ is the number of atoms and $\mathrm{P}$ is the number of processors. The neighbor listing could be modified by defining certain parameters. We choose not to delay for building the new neighbor list at all after the defined number of time steps are completed from the initial building of neighbors. We have also checked before creating the new neighbors whether some of the atoms moves more than half of the skin distance or not.

Rigid bodies are consists of several atoms but it considers all force and torque applied on it as sum of forces and torques in its constituent particles. Therefore despite having several particles the whole structure acts as one body particle [52]. Protein, colloidal particles are the examples of rigid bodies. The star particles we have generated for our simulation was consists with small 
circular particles. So while running the simulation we considered those constituent particles together as rigid body. Also as we used multiple star particles in each system therefore the body style was set as molecule for each rigid bodies.

Our simulation box is has very small dimension in $\mathrm{z}$ axis compared to the other two dimension. Therefore, we could consider our simulation as a $2 \mathrm{D}$ case and take care about the dynamics so that it zero out the velocities and forces for each of the atoms in simulation in $\mathrm{z}$ direction. For each time steps we computed and dumped our desired parameters such as shear stress, strain and pressure values. But to check whether the dynamics is maintaining the desired behavior and following the conservation law we have also dumped the kinetic energy, potential energy, temperature, velocity and the values of defined variables at each time step.

The visualization is also an important part in case of MD simulation. We have dumped the coordinates of each of the atoms after a certain time steps in xyz format and loaded the dumped file in VMD [53] to visualize the simulation.

\subsection{Star particle Generation}

We have generated functional star particles with asperities to infuse into the fluid. For this purpose we have used an algorithm using python programming language. Our goal was to generate particles with arms. Also to create the asperity [18] on the surface we decided to use small spherical/circular particles and by joining those particles create our desired star shaped particles. The small circular particles that we chose has radius $r=0.2 \sigma$. We compiled few of those small particles together to form the core of the star particle. The radius of the core $\mathrm{R}$ was chosen $0.65 \sigma$. We run 1000 trials to place the particles in the center so that these placed randomly with no overlap and also make sure these particles are not too close. After creating the core decide the number of leg we add using the following formula,

$$
\theta=2 * \frac{\pi}{\text { Nleg }}
$$

Where $\theta$ the angle between legs and Nleg is the number of legs. In our case we choose Nleg equals 3, 5 and 7 . The number of particles in each leg is determined as the following, 


$$
n=\frac{L}{2 * r}+1
$$

Here $\mathrm{L}$ is leg length and $\mathrm{n}$ is the number of small particles in each leg. However we set this formula such a way that only took the integer value of the first term of above equation. The radial distance from the center of core to the edge of leg is calculated afterwards as the following way,

$$
\begin{aligned}
& r_{d}=(R+r) * \operatorname{Cos}(\theta * i) \\
& r_{d}^{a}=r_{d}+j * 2 * r * \operatorname{Cos}(\theta * i)
\end{aligned}
$$

Here $r_{d}$ is the radial distance from core, $r_{d}{ }^{a}$ is the distance where the center of newly added small particle is placed and $\mathrm{i}$ and $\mathrm{j}$ are the iteration numbers which determines how many number of legs are creating and how long the leg lengths are extending respectively.

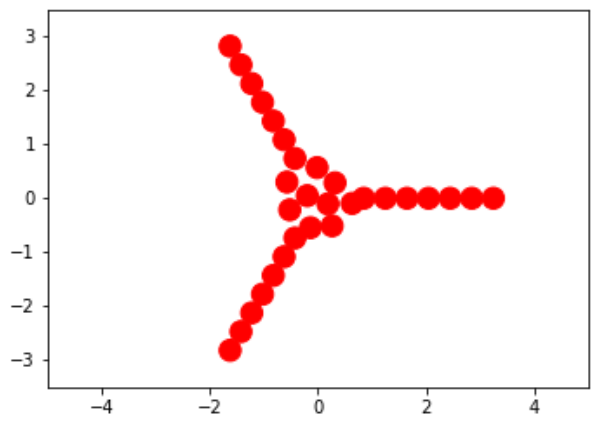

(a)

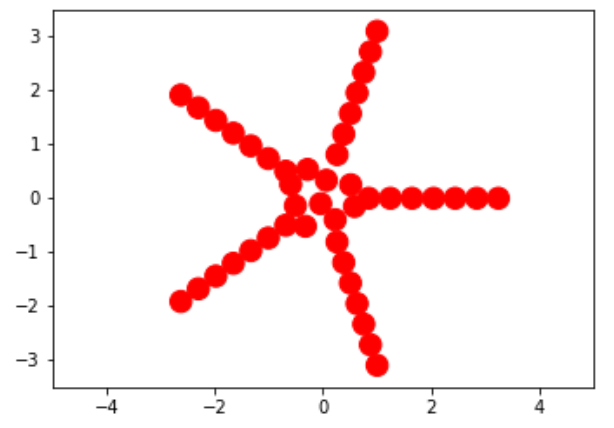

(b)

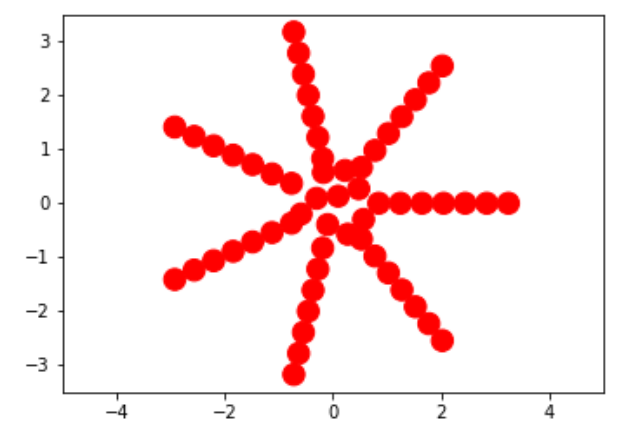

(c)

Figure 3.1: Single (a) 3leg (b) 5leg and (c) 7leg Star particle with core radius $0.65 \sigma$ and leglength $2.5 \sigma$. 
We have created 30 star particles for each system having same number of legs and leg lengths and dispersed those into a $30 * 30$ square unit area randomly. We calculated the packing fraction $(\varphi)$ of the system using the following way,

$$
\varphi=\frac{\text { Number of star particle } * \text { area of each particle }}{\text { Total area of simulation box }}
$$

We tried to cover the range of packing fraction starting from close to 0 and ending close to 1.As leg length is the only variable here that could vary this packing fraction we choose leg length equals $0.0,0.5,1.0,1.5,2.0,2.5,3.0,3.5,4.0,4.5,5.0,5.5,6.0$ for 3 leg that gives us packing fraction equals $0.18,0.23,0.28,0.34,0.44,0.49,0.54,0.59,0.69,0.74,0.79,0.84$, 0.94 respectively. And leg length $0.0,0.5,1.0,1.5,2.0,2.5,3.0$ for 5 leg that gives the packing fraction $0.22,0.30,0.40,0.47,0.62,0.72,0.80$ respectively. Finally leg length $0.0,0.5,1.0$, $1.5,2.0,2.5$ for $7 \mathrm{leg}$ that gives the packing fraction $0.25,0.37,0.50,0.60,0.84,0.96$ respectively.

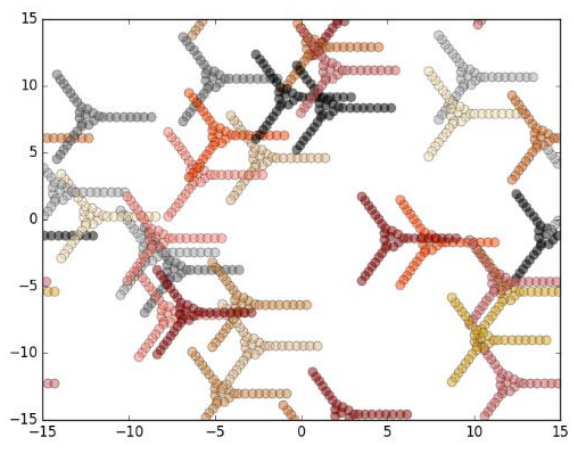

(a)

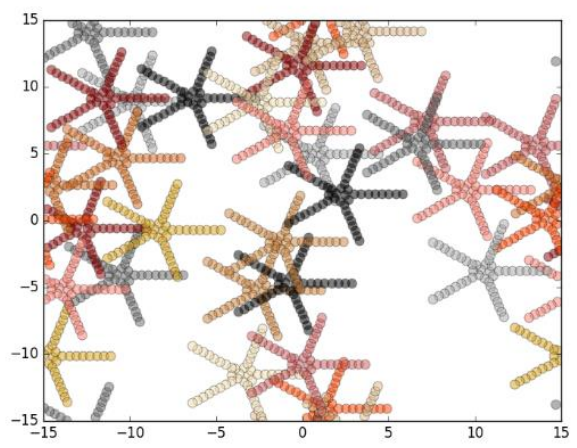

(b)

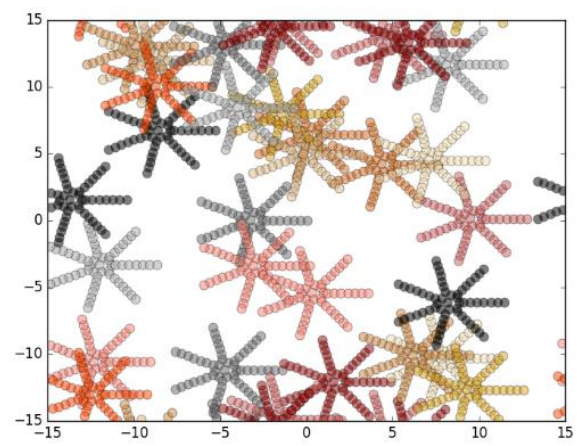

(c)

Figure 3.2: Randomly dispersed configuration of 30 Star particles in each system having a square shaped (30*30 $\sigma^{2}$ ) simulation box for (a) 3leg (b) 5leg and (c) 7leg. 
To do an experimental demonstration and compare the simulations with real world experimentation we have created few physical star particles by 3D printing and Laser cutting. For this purpose first we designed the particle with one of the popular engineering design software named Solidworks. We choose the Acrylonitrile butadiene styrene (ABS) plastic and 3/16" x 24" x 24" - Orange Plexiglas Acrylic Sheet as the material for completing our 3D printing and LASER cutting respectively. As the dimensions are convertible from reduced unit to any physical unit we could easily transform all the dimensions in physical form and perform the similar procedure to rise the chosen fluids viscosity in a long rectangular pipe by experimentation. The dimensions of one particular star particle having a circular core with radius $0.65 \sigma$ and leg length $2.5 \sigma$ is printed for experimental demonstration by transforming into physical unit. We have printed 5 legged star particles with core radius $0.65 \mathrm{~cm}$ and each leg length equals to $2.5 \mathrm{~cm}$. Each leg has given a width of $0.4 \mathrm{~cm}$. The thickness of star particles are kept $0.45 \mathrm{~cm}$ which is very small compared to the length and width of the system and thus creates an almost 2D case like our simulation. Around 30 particles are printed for a system with control volume $30 \mathrm{~cm} \times 30 \mathrm{~cm} \times 0.5 \mathrm{~cm}=450 \mathrm{~cm}^{3}$.

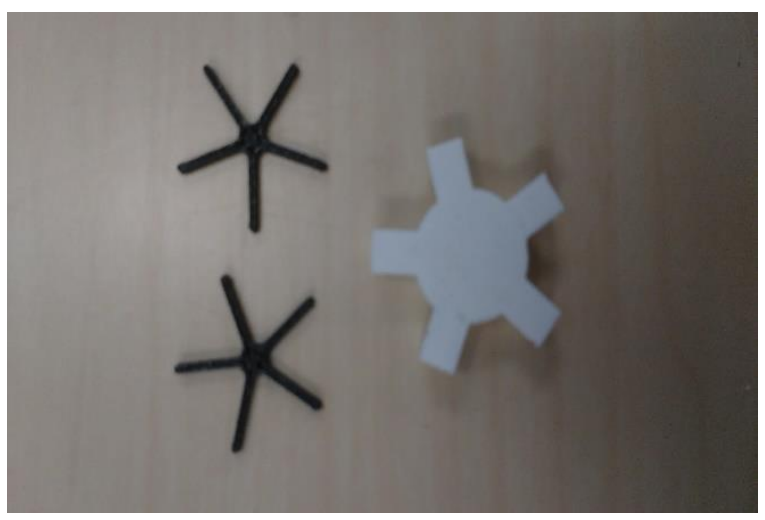

(a)

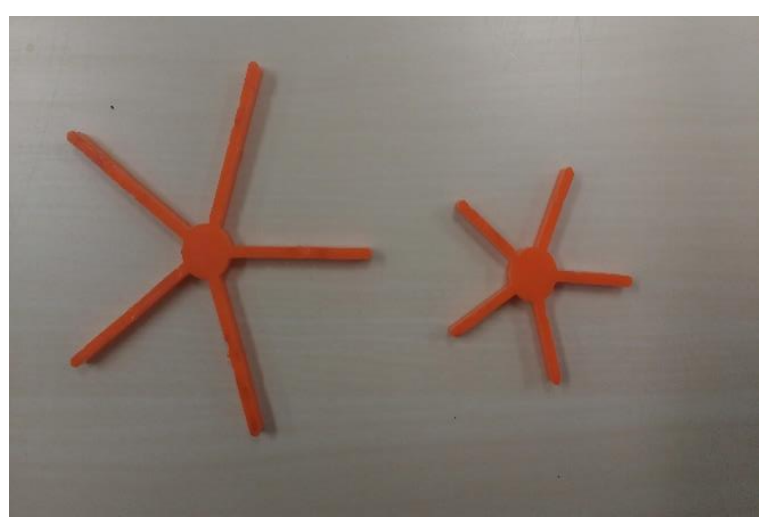

(b)

Figure 3.3: 5 leg star particles by (a) 3D printing and (b) LASER cutting.

\subsection{Stochastic Rotation Dynamics (SRD)}

Stochastic Rotation Dynamics (SRD) [54]-[60], introduced by Anatoly Malevanets and Raymond Kapral, are cheap-coarse grained particles that serves as background solvent that do 
not interacts with each other but with solute particles of different shapes (spheroid, ellipsoid, line, circular, triangle, rigid bodies etc.). The fluid like property of SRD imbues due to the collision performed between SRD and solute particle and the random rotation that sets the velocity distribution after each number of fixed time steps. As SRD's do not interact with each other therefore the large scale calculation becomes possible for calculating the solute properties (i.e. viscosity, diffusivity) in background fluid. The mass of each SRD could be set in the input script. The mass of SRD's $\left(m_{S R D}\right)$ could be related with the temperature (T), SRD timestep $(\Delta t)$ and mean free path $(\lambda)$ between collisions of SRD particles with a fundamental equation as following [61],

$$
\lambda=\Delta t \sqrt{\frac{k_{B} T}{m_{S R D}}}
$$

Where $k_{B}$ is the Boltzmann constant. The SRD time step slightly different than the simulation time step. When the suspended solute particles advect with time step dt, the SRD particles advect with $\mathrm{N}^{*} \mathrm{dt}$ where $\mathrm{N}$ is an integer number of time step of the simulation. Therefore SRD time step is higher than the solute time step and that is why they have different timescale. The random rotation takes place after this SRD time steps but the collision steps follow the simulation time steps. The length scale of SRD to update velocities is set equal or lower than the one-fourth of the solute particles diameter to adequately create the fluid like property around the solute. Usually the SRD particles have less mass than the solute particles and therefore in the collision steps when SRD's collide with solutes it bounces back with new velocity that changes the momentum of SRD's. This collision also causes the force and torque that imparts in the solute particle. The style of collision could be set in two ways. One is slip and other is no slip. Slip condition would impart only force but no torque in the solute particle. But no slip will impart both. The solute particle overlapping could also be controlled. No overlap could fast the collision calculation process. The simulation creates two kinds of bins SRD bin and Search bin. In SRD bin it is used to bin the SRD particles and update their velocities. The search bin is used for collision of SRD and solute particle calculations. The bin size almost equal to the SRD length scale and it could vary to adjust the number of bin as an integer value. The random shifting of all particles with same vector in a bin is made while the particles does not moves much higher than the mean free path in the rotation step for velocity update. During the collision of SRD and solute particle, the stochastic temperature control method is implemented in the bins. Without any thermostat it will take much long time for the 
SRD's to come in equilibrium state. The maximum velocity of SRD particles could also be controlled.

SRD is coarse-grained particle based method [62] that has numerical stability, internal thermostatting and simple implementation. It could also be used in fluctuation fluid dynamics to address sedimentation, reacting fluid, vesicles in flow, polymers in flow etc [58]. The details are not fully solved but it could provide correct hydrodynamic interaction among embedded particles [60]. SRD works better when the Peclet number is with order 1 and Reynolds numbers in between 0.05 to 20 . Although SRD method is much faster, it could not be used for arbitrarily large peclet numbers. For that reason the amplitude of noise should be reduced. If a large number of particles are used in each node that would reduce the noise. But increasing the particle number at each node does not work for peclet number higher than 20. Also for high Reynolds number this method is inefficient. The $\mathrm{H}$ theorem shown that this method has correct hydrodynamic equations [63].

For a system with coarse grained particles placed in square shaped lattice then the system is divided into a number of bins. The method actually consists of two distinct steps. One is Collision step and the other is Streaming step. In the streaming step the particles updated its positions during each time step. The position is updated according to its displacement vector in each SRD time step. Collisions step is performed by random rotation matrix. It updates the particles velocity at each SRD time steps. During collision steps the rotation matrix performs in each bin separately. It conserves the mass, momentum and energy consistent with the conservation laws at collision steps.

For a system that consists $\mathrm{N}$ number of point SRD particles having position $r_{i}(\mathrm{t})$ and velocity $v_{i}(t)$ for $i$ th particle at time $\mathrm{t}$ we could figure out the position of and velocity of that particle after small SRD time step dt. In the streaming step the particle having a velocity $v_{i}(t)$ will move a distance equals $v_{i}(t) * \mathrm{dt}$. That would give its new position [58],

$$
r_{i}(\mathrm{t}+\mathrm{dt})=r_{i}(\mathrm{t})+v_{i}(t) * \mathrm{dt}
$$


Again for collision step particles of each bin are interacts. For random rotation matrix $\omega(\xi)$ where $\xi$ is the bin number cause the reset of particles velocity in that particular bin. The relative velocity is $v_{i}$-u where $\mathrm{u}(\xi, t)$ is the mean macroscopic velocity of the particles in the bin $\xi$. The velocities are updated after dt timesteps as followings [58],

$$
v_{i}(\mathrm{t}+\mathrm{dt})=\mathrm{u}\left[\xi_{i}(\mathrm{t}+\mathrm{dt})\right]+\omega[\xi(t+d t)]\left\{v_{i}(\mathrm{t})-\mathrm{u}\left[\xi_{i}(\mathrm{t}+\mathrm{dt})\right]\right\}
$$

The local temperature $\mathrm{T}(\xi, t)$ is defined by the mean square displacement of the particles velocity from the mean velocity $\mathrm{u}$ at the bin $\xi$. The mean velocity $\mathrm{u}$ is the average velocity of all particles inside the bin $\xi$ which is calculated as following [58],

$$
\mathrm{u}(\xi)=\frac{1}{N} \sum_{k \in \xi} v_{k}
$$

If the square shaped bin size has side length $\mathrm{b}$ then for removing the temperature anomalies before the collision step the particles are shifted by same random vector ranging from $\left[-\frac{b}{2}, \frac{b}{2}\right]$.

\subsection{Observables: Viscosity, Diffusivity, Radial Distribution Function}

Viscosity is the characteristic property of fluid. It is basically the resistance that a fluid face while trying to flow. Viscosity $\eta$ is expressed as,

$$
\eta=\frac{\mathrm{F} / \mathrm{A}}{\frac{\partial u}{\partial y}}=\frac{\tau}{\frac{\partial u}{\partial y}}
$$

Where $\mathrm{F}$ is the force parallel to a surface with area $\mathrm{A}$ and $\frac{\partial u}{\partial y}$ is the velocity gradient.

It is also called the dynamic viscosity or absolute viscosity or shear viscosity [64]. The SI unit of viscosity is $\mathrm{Pa} / \mathrm{s}$. Poise is another unit of viscosity where $10 \mathrm{P}=1 \mathrm{~Pa} / \mathrm{s}$. There is another quantity that is called kinematic viscosity which is just the ratio of viscosity $\eta$ to density . This is a measure of fluid flow under the influence of gravity. The SI unit of kinematic viscosity is $\mathrm{m}^{2} / \mathrm{s}$. Stokes is another unit of kinematic viscosity where $10^{4}$ stokes = $1 \mathrm{~m}^{2} / \mathrm{s}$.

$$
v=\frac{\eta}{\rho}
$$


Viscosity varies fluid to fluid. The more the viscosity more thick and resistive the fluid is. Like water has less viscosity than honey and therefore water is thinner and it flows more freely than honey does. Viscosity of fluid is normally the friction between the fluid molecules. So when the molecules come closer to each other this friction and so does the viscosity increases. Viscosity mostly depends on temperature. When the temperature increases the molecules goes far from each other which cause the viscosity go down and opposite when the temperature decreases. The same thing is not true for Pressure because incompressible fluids could not force the molecules to come closer to each other to cause more friction in between them and therefore no change in viscosity. But if the fluid compresses due to high pressure then the viscosity will rise. As the viscosity is dependent on temperature therefore it is required to mention the viscosity value of particular fluid with respective temperature.

The fluids are also characterized into two categories named Newtonian fluid and NonNewtonian fluid. Newtonian fluid follows a linear relationship with shear stress and strain rate. And Non-Newtonian fluid follows a non-linear relation with shear stress and strain rate. Also there is a concept of Bingham plastic which has a linear relationship like Newtonian fluids except that it requires to pass an initial shear stress barrier to start flowing like fluid. And Bingham pseudo plastic also needs to pass in initial shear stress barrier but the relationship is non-linear. In general, all of the fluids are non-Newtonian fluid. Two new categories of fluid exists under non-Newtonian fluid those are shear-thinning fluid and shear thickening fluid. If the viscosity of the fluid increases with respect to the increase of strain rate then the fluid is called shear thickening fluid. And if the viscosity decreases with the increase of shear rate then it is called shear thinning fluid. The velocity profile varies depending on what kind of fluid we have. 


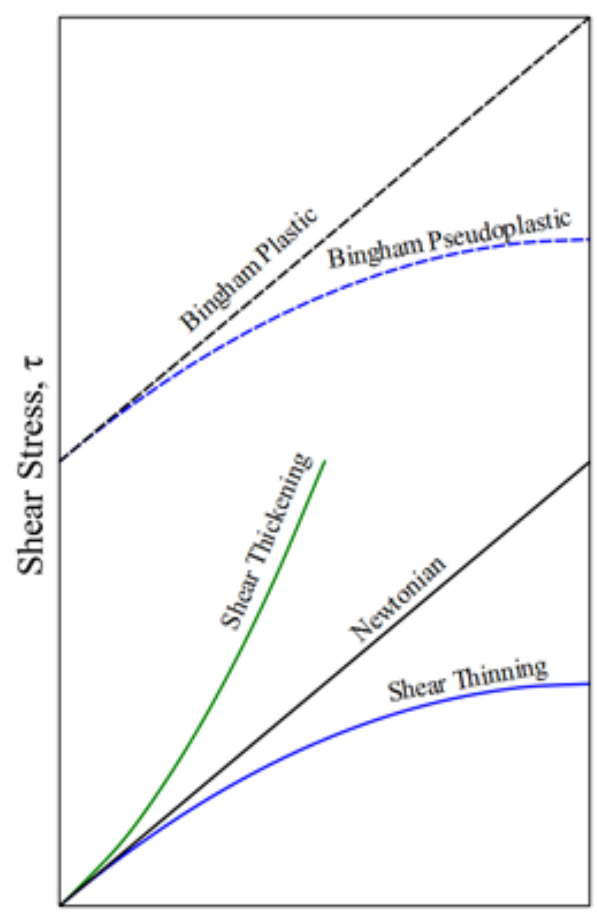

Shear Rate, $\dot{\gamma}$

(a)

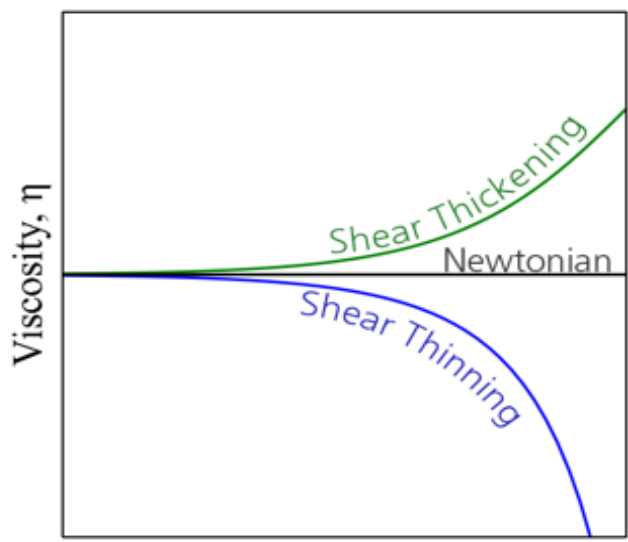

Shear Rate, $\dot{\gamma}$

(c)

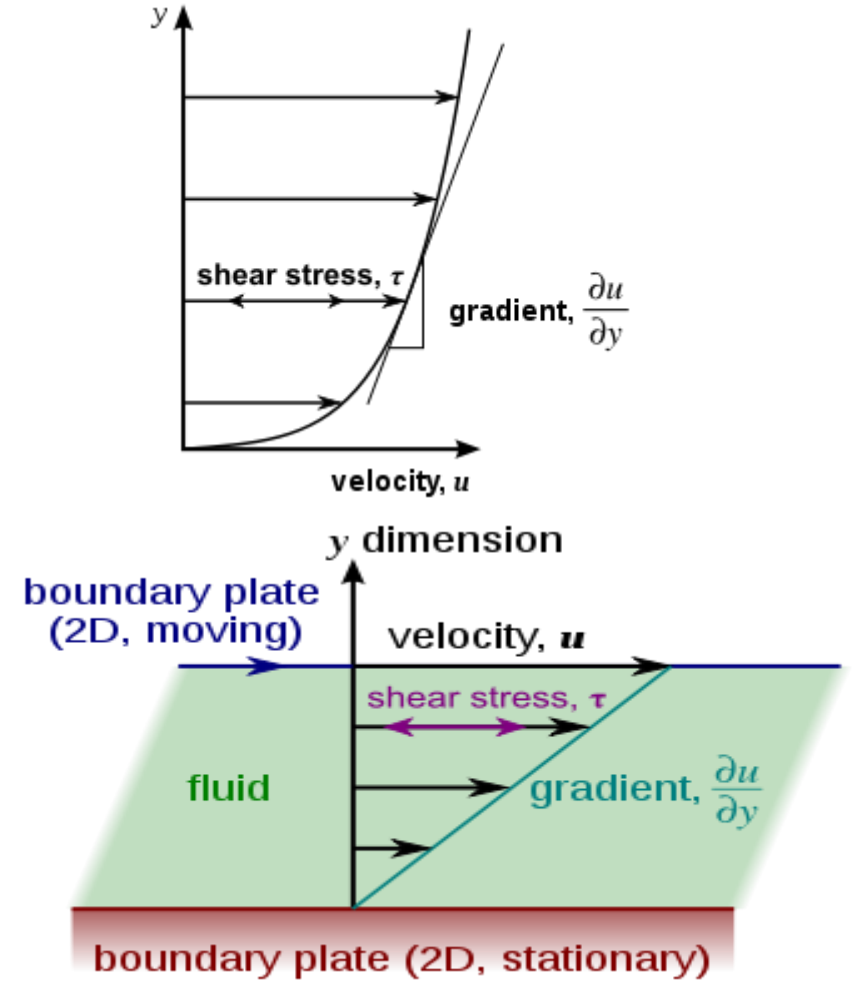

(b)

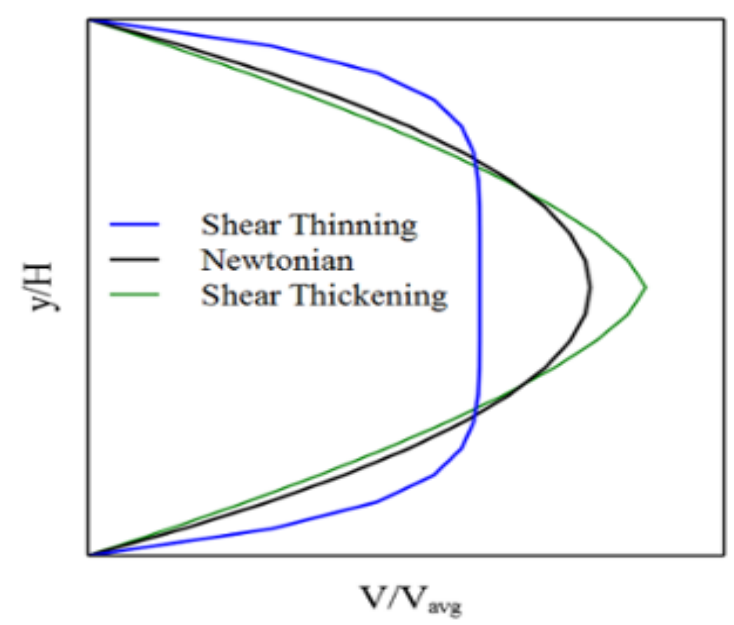

(d)

Figure 3.4: (a) Shear stress as a function of shear rate for different Newtonian, Non-Newtonian shear thinning and shear thickening, Bingham plastic and Bingham Pseudoplastic fluid (b) shear stress on a fluid in between two boundary plate where one is moving and another is stationary (c) Viscosity of Newtonian, Shear thinning and shear thickening fluids as a function of shear rate (d) velocity profile for Newtonian, shear thinning and shear thickening fluid [65] 
Viscosity can be measured both experimentally and computationally. Some of the experimental method to measure viscosity are Capillary viscometer, Zahn Cup, Falling Sphere Viscometer, Vibrational Viscometer, Rotational Viscometer etc. [66][67]

Typically in simulations, the rheological properties such as viscosity of fluids are measured by Non-equilibrium molecular Dynamics (NEMD) simulations [68]-[71]. A non-orthogonal simulation box needs to set up for running NEMD simulation where shear strain is applied with a desired constant strain rate. Although reverse Non-equilibrium Molecular Dynamics (rNEMD) [72] and equilibrium Molecular dynamics could also determine fluids viscosity. LAMMPS could use any of the MD, NEMD or rNEMD method to measure the fluids viscosity by the following five ways. A brief discussion regarding all of the methods are described below.

Non Equilibrium Molecular Dynamics (NEMD) simulation deforms the simulation box using fix deform command with constant engineering or true strain rate in LAMMPS. The other method is named as Wall shearing also follows the same NEMD simulation technique but in this case shear is applied by moving one or several walls. This NEMD method runs based on the cause and effect. The velocity gradient or strain rate is the cause and its effect is found as the momentum flux or stress. The viscosity is calculated from the ratio of transferred momentum perpendicular to the velocity direction to the gradient of velocity. For flow in $\mathrm{x}$ direction and transfer of momentum in $\mathrm{z}$ direction the momentum flux $j_{z}\left(p_{x}\right)$ and velocity gradient $\partial v_{x} / \partial z$ is expressed as [72],

$$
j_{z}\left(p_{x}\right)=-\eta \frac{\partial v_{x}}{\partial z}
$$

Where $\eta$ is the proportionality constant otherwise known as shear viscosity. The velocity is set up in the simulation initially by ave/chunk that determines the velocity gradient. And pressure tensor $P_{x z}$ is calculated by compute pressure command in LAMMPS. This $P_{x z}$ is the $\mathrm{x}$ component of momentum $p_{x}$ transported along $\mathrm{z}$ direction per unit time and unit area.

$$
j_{z}\left(p_{x}\right)=\frac{p_{x}}{2 t A}
$$

Here $\mathrm{t}$ is the time and $\mathrm{A}$ is the area. Because of periodicity we had to divide the momentum component by a factor 2 . 
The rNEMD simulation method is almost similar to the NEMD method but in this case the cause and effect that we discussed earlier for NEMD simulation is reversed. Instead of applying velocity gradient or shear rate rNEMD method imposed momentum flux or stress that cause the velocity gradient or strain rate. The momentum flux is imposed by swapping momentum in one direction for atoms in different layers and different direction. The swap of momentum induces velocity gradient that is monitored by the same way as NEMD. This is generally known as Muller-Plathe algorithm [72]. LAMMPS has separate command names fix viscosity to calculate viscosity with this rNEMD method.. This method is also known as Norton-ensemble methods.

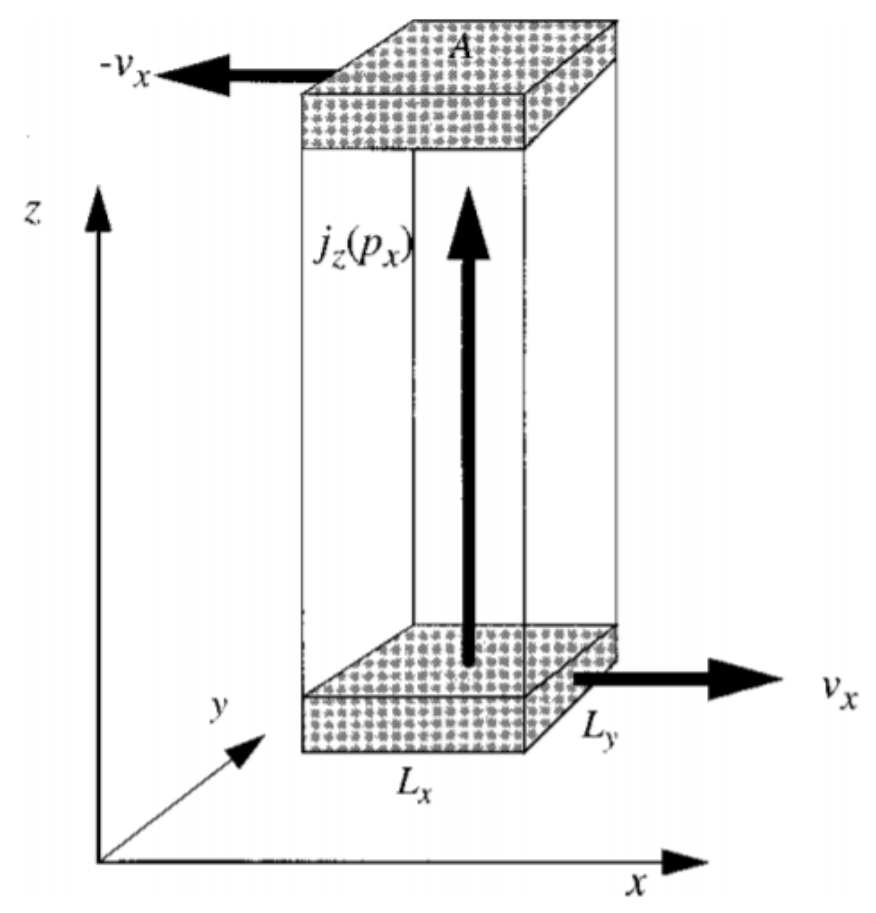

Figure 3.5: Nonequilibrium simulation geometry. Velocity gradient in $v_{x}$ is set up in the $\mathrm{z}$ direction by shearing the liquid. And $\mathrm{x}$ momentum flows towards $\mathrm{z}$ direction that rise momentum flux $j_{z}\left(p_{x}\right)$ through the xy plane. [72]

The simulation methods mentioned earlier calculates the viscosity of fluids directly and gives the viscosity value as output. We have followed the same NEMD technique but instead of getting the direct viscosity value we have dumped the viscous shear stress. Then using its relation with shear strain rate we measured the slope of their plotting to get shear viscosity. 
Viscous stress tensor describes the internal forces in fluids. It is the results of strain rate (rate of change of deformation with respect to time). It actually arises from the friction or particle diffusion of the fluid particles of a system with different mean velocities. On the other hand elastic stress tensor describes the internal forces in elastic material because of deformation. Due to the jamming transition, our system transformed from liquid-like state to an intermediate state between solid and liquid therefore ours one is actually viscoelastic system that has both viscous and elastic component in its total stress tensor.

The viscous stress tensor in a 3D could represented as a 3x3 matrix in following way at any point $r$ with time $t$,

$$
\varepsilon(r, t)=\left|\begin{array}{lll}
\varepsilon_{x x} & \varepsilon_{x y} & \varepsilon_{x z} \\
\varepsilon_{y x} & \varepsilon_{y y} & \varepsilon_{y z} \\
\varepsilon_{z x} & \varepsilon_{z y} & \varepsilon_{z z}
\end{array}\right|
$$

If there is any torque applied by any external source like external magnetic fields in case of Magnetorheological fluids or external electric fields in case of electrorheological fluids the viscous stress tensor will be antisymmetric. As we have not applied any external force fields in our system our stress tensor should be symmetric and therefore we got $\varepsilon_{x y}=\varepsilon_{y x}, \varepsilon_{x z}=\varepsilon_{z x}$ and $\varepsilon_{y z}=\varepsilon_{z y}[73]$.

As our system is viscoelastic therefore total stress will be the sum of viscous stress tensor and elastic stress tensor which manifests itself as hydrostatic pressure p. Therefore the total stress could be expressed as following,

$$
\sigma_{i j}=-p \delta_{i j}+\varepsilon_{i j}
$$

Where $\delta_{i j}$ is unit tensor such that $\delta_{i j}=0$ if $\mathrm{i} \neq \mathrm{j}$ and $\delta_{i j}=1$ if $\mathrm{i}=\mathrm{j}$.

The strain rate tensor $\mathrm{E}(\mathrm{r}, \mathrm{t})$ is the derivative of strain tensor $\mathrm{e}(\mathrm{r}, \mathrm{t})$ with respect to time. As it is the rate at which the mean velocity in the medium changes so strain rate tensor (represents the velocity pattern) could be expressed with respect to the gradient of velocity vector $v(r, t)$ also. The expression could be written as,

$$
E_{i j}=\frac{\partial e_{i j}}{\partial t}=\frac{1}{2}\left(\frac{\partial v_{j}}{\partial x_{i}}+\frac{\partial v_{i}}{\partial x_{j}}\right)
$$

Strain rate tensor and viscous stress tensor represents the velocity pattern and viscous stresses at any point $\mathrm{r}$ are related by viscosity tensor $\mu$ as following, 


$$
\varepsilon_{i j}=\sum_{k l} \mu_{i j k l} E_{k l}
$$

Here the viscous stress tensor could be expressed as the sum of symmetric tensor $\varepsilon^{S}$ commonly known as viscous shear stress related with shearing deformation and scaler multiple $\varepsilon^{v}$ of identity tensor when the material is compressed or expanded at same rate. The bulk viscosity $\mu^{v}$ is the slope found from stress tensor $\varepsilon^{v}$ vs strain rate tensor $E^{v}$ whereas shear viscosity $\mu^{S}$ is the slope found from stress tensor $\varepsilon^{S}$ and strain rate tensor $E^{S}$. Mathematically these quantities are related as following,

$$
\begin{gathered}
\varepsilon_{i j}=\epsilon_{i j}^{v}+\epsilon_{i j}^{S} \\
\epsilon_{i j}^{v}=\frac{1}{3} \partial_{i j} \sum_{k} \varepsilon_{k k} \\
\epsilon_{i j}^{S}=\varepsilon_{i j}-\frac{1}{3} \partial_{i j} \sum_{k} \varepsilon_{k k} \\
\varepsilon^{v}=\mu^{v} E^{v} \\
\varepsilon^{s}=\mu^{s} E^{s}
\end{gathered}
$$

We have calculated the viscous shear stress $\epsilon_{i j}^{S}$ from our simulation and calculated the shear viscosity $\mu^{s}$ by calculating the slope for given strain rate tensor $E^{s}[74]$.

We have also calculated the standard error of mean by considering the values we found from configurations of same leg lengths for specific number of legs for plotting the error bar. The standard error of mean is calculated by the following formula,

$$
S E M=\frac{\sigma}{\sqrt{n}}
$$

Where, SEM= standard error of mean, $\sigma=$ standard deviation of the samples and $\mathrm{n}=$ number of sample used.

Diffusion is associated with the random thermal motion of atoms that changes the macroscopic concentration profile. This process occurs in gases liquids amorphous and crystalline solids of metals, ceramics, polymers, semiconductors etc. It is the net movement of atoms or molecules from high concentration to low concentrations. The atomic structure of materials along with any defects in it such as rates of kinetic processes, processes that modify materials and material failure etc. could be investigated by diffusion. It is solely dependent on Temperature. Diffusion follows the Random walk model that means that the particles moves randomly without 
knowing the path it is going to take. The diffusion equation in 3D which is also known as Fick's $2^{\text {nd }}$ law is written as the following [75],

$$
\frac{\partial c}{\partial t}=D\left(\frac{\partial^{2} c}{\partial x^{2}}+\frac{\partial^{2} c}{\partial y^{2}}+\frac{\partial^{2} c}{\partial z^{2}}\right)
$$

Where $\mathrm{c}$ is the concentration and $\mathrm{D}$ is the diffusivity which is our prime interest.

Diffusivity is the rate of diffusion at which particles or heat spreads. It is also known as diffusion coefficient. It. The diffusivity of particles are known as mass diffusivity. Similarly, diffusivity of heat is known as thermal diffusivity. In our latter discussion we will be focusing on mass diffusivity only.

Diffusivity of mass could be categorized into three types. First one is self-diffusion coefficient which is the rate of diffusion of atoms which are labeled. As the atoms are literally indistinguishable from one another this kind of diffusivity could be calculated using radioactive isotopes. The second type is hetero-diffusion coefficient which refers to the rate of diffusion of one molecular species into another. This type of diffusion needs more than one type of molecules and most of the diffusions in our practical life actually falls into this category. The final type is mutual diffusion coefficient. When different molecule types diffuses into each other then the rate which this mutual diffusion is occurred is called mutual diffusion coefficient.

Diffusivity closely connected with mean square displacement (MSD) also known as average squared displacement or mean square fluctuation. It measures the deviation of particles position from a reference point with respect to time. Mathematically it can be described as the following equation,

$$
M S D=\overline{\left(x-x_{0}\right)^{2}}=\frac{1}{N} \sum_{n=1}^{N}\left(x_{n}(t)-x_{n}(0)\right)^{2}
$$

Where $\mathrm{N}$ is the number of particles, $x_{n}(0)$ is the reference position and $x_{n}(t)$ is the position after time t. As in random walk series of steps are taken randomly so if it moves forward and then comes back to its initial position then by adding its vector sum of distance will give zero value that is no use to calculate the total distance travelled. Therefore MSD is much appropriate to use for calculating total distance because it squares each distance and sums up all the positive values and finally gives the average. The relation of MSD and Diffusivity is analyzed by Einstein in his study of Brownian motion and he showed that MSD is proportional to the time elapsed which is related as the following equation [76], 


$$
M S D=6 D t+C
$$

Where $\mathrm{t}$ is the time and $\mathrm{D}, \mathrm{c}$ are constants. This $\mathrm{D}$ is the diffusion coefficient and hence we could find this quantity from the slope of MSD vs time plotting. Although at very short time this relationship is not linear rather it is parabola. The reason is the relationship holds a linear relationship when the path particles follows is completely random and randomness is generated by collisions of particles with its neighboring particles. But at very short time before making any collisions the particle moves with constant velocity and therefore the distance it travels is proportional to time and MSD becomes proportional to time squared.

For diffusion coefficient calculation of our system we calculated the mean square displacement of group of atoms by considering the periodic boundaries. We have two groups of particles in our system. One is the small SRD particles and other one is the big star particles. First we calculated the displacement in all direction $\mathrm{dx}, \mathrm{dy}, \mathrm{dz}$ and after that total squared displacement $(d x * d x+d y * d y+d z * d z)$ is calculated. Then the sum of all the total displacements for each atom is divided by the total number of atom to get the mean squared displacement (MSD). The slope of mean squared displacement vs time step plot gives the diffusion coefficient of those atoms. We run our simulation for a longer time period so that the relationship truly becomes linear. For calculating the diffusion coefficients of molecules (star particles in our case which is composed of several number of atoms) we calculated the center of mass for each molecule and then considered those center of atoms and followed the same procedure for diffusion coefficient [77].

Radial distribution function (RDF) is also known as Pair correlation function $\mathrm{g}(\mathrm{r})$ which describes how the particles dispersed in a system from by taking account of one reference particle. In simple it is the probability of finding a particle from a reference particle at specific distance. It could describe the structure of molecular system effectively. Liquid is a disordered molecular system whose molecules are moving continuously. The snapshot of any time could capture the instantaneous disorder in the liquid which could be used for calculation of average structure of that system.

The usefulness of RDF calculation is vast. The experimental studies with various method such 
as X-ray, Neutron diffraction etc. could easily be compared with simulation methods result using this RDF method. It also could calculate the internal energy almost accurately by combining with interatomic pair potential function. It could also be used to calculate the Structure factor [78].

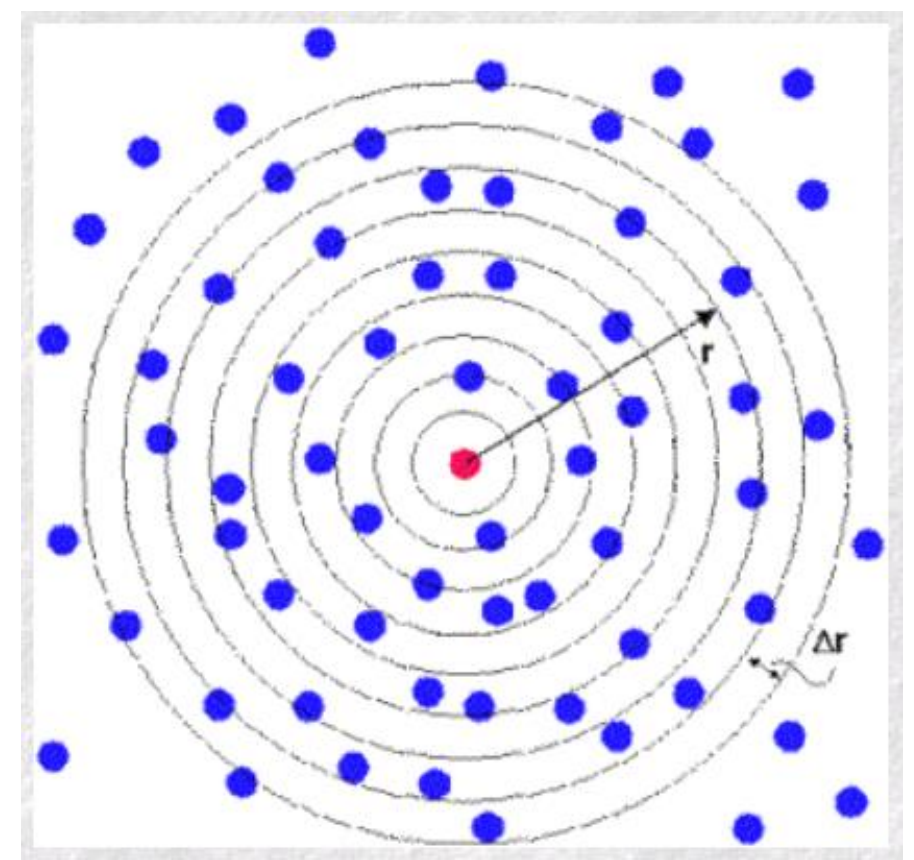

Figure 3.6: Calculation of radial distribution function $\mathrm{g}(\mathrm{r})$. Red particle is the reference particle and blue particles falls within the range of radial increment $\Delta \mathrm{r}$ lined in black [78]

RDF can be easily constructed for a simulation for better understanding of this method. Select a system with dispersed particles that are moving while the simulation is running. Now choose one specific particle near the center of the system and draw concentric spheres (for 3D) or circles (for 2D) around it with an increment of $\Delta \mathrm{r}$ from each other. Now for a particular time calculate the number of particles at each shell. It is better to take snapshots of that particular system to ensure no particle moves during this calculation. Follow this procedure a number of times with a fixed interval. Finally take the average number of atoms in each shell. Now RDF is the ratio of the average number of atoms in each shell to the volume (for 3D) or area (for 2D) of each shell multiplied with the average density of particles in the system. Mathematically, Radial distribution function $\mathrm{g}(\mathrm{r})$ is, 


$$
g(r)= \begin{cases}\frac{n(r)}{\rho 4 \pi r^{2} \Delta \mathrm{r}} & , \text { for } 3 D \\ \frac{n(r)}{\rho 2 \pi r \Delta \mathrm{r}} & , \text { for } 2 D\end{cases}
$$

Where average atom density $\rho$ is defined as,

$$
\rho= \begin{cases}\frac{N}{\text { Volume }}, & \text { for } 3 D \\ \frac{N}{\text { Area }}, & \text { for } 2 D\end{cases}
$$

Here $\mathrm{N}$ is the total number of particles and $\Delta \mathrm{r}$ is the shell width at distance $\mathrm{r}$. We could also choose each different atom from the system separately and follow the same procedure to calculate RDF that would lead to the average RDF calculation for the system.

The RDF calculation makes more sense when it is plotted with the radial distance $r$. This plot has some important aspects. First is, for very small $r$ value the RDF is zero because it indicates the width of the reference particle and there is no other particle in its width unless overlapping is allowed. Second important feature is couple of peaks. The first peak is highest for most of the cases. This peaks indicates that the packing of atoms in reference to its neighborhood. The higher the number of peaks the higher the degree of ordering. Usually the highest peak comes first and then the rest of the peaks decreases as sequentially as the radial distance increases and finally it reaches to a value of 1 which is because it means the average density as a whole. 


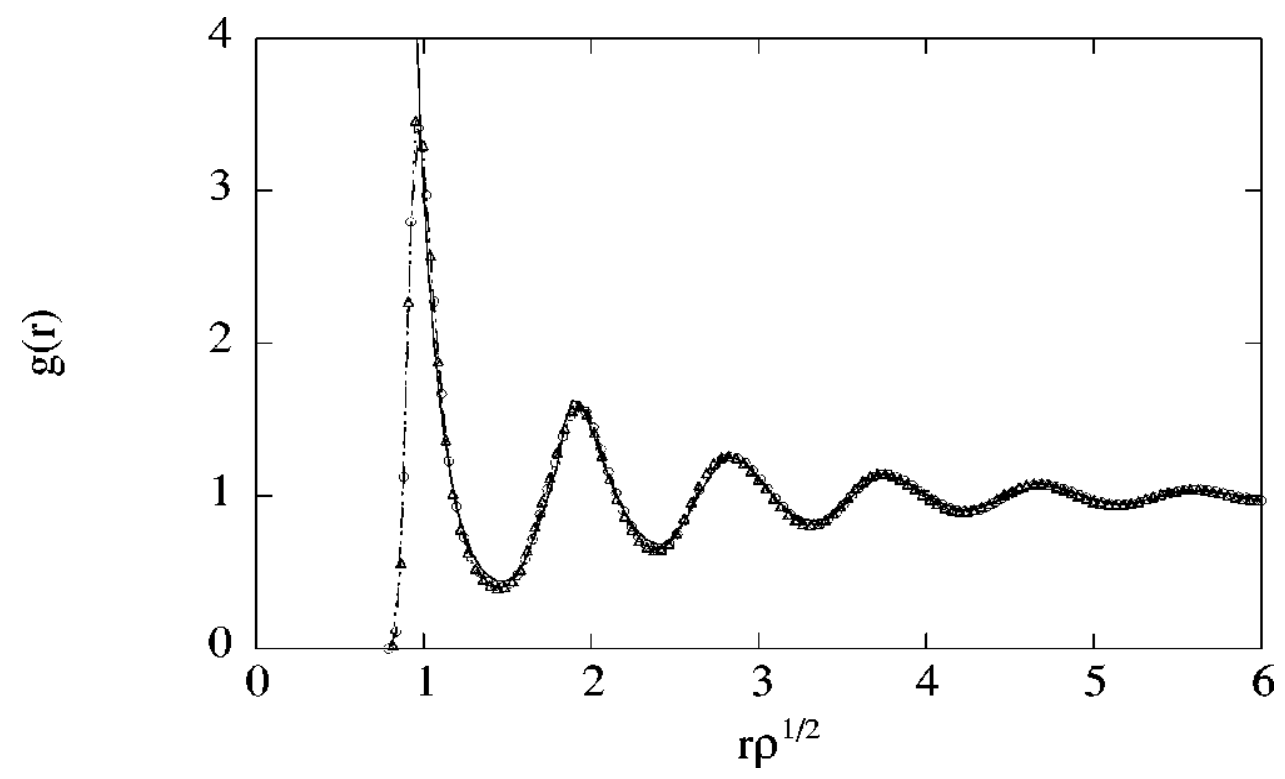

Figure 3.7: Radial distribution function for the Lennard-Jones fluid model [79]

We have various systems of star particles with different number of legs and leg lengths. Each system also has 30 different configurations of dispersed stars. We have calculated the radial distribution function $\mathrm{g}(\mathrm{r})$ for each system configurations and then averaged over the configurations to get statistically precise plotting. For these calculations we have used $\Delta \mathrm{r}=0.2$ unit and maximum radial distance $\mathrm{r}=10$ unit.

\subsection{Boundary Conditions}

A flow model can describe rheological data (i.e. shear stress, strain rate, packing density etc.) in mathematical form. That equation could further use for plotting the flow as a basic diagram as a concise way to describe the flow. Moreover the variation of model parameters due to state variables could also be understood more conveniently by using functional models [80]

There are 3 types of functional models for rheology: 1. Empirical 2. Theoretical and 3. Structural. Empirical model is deduced from experimental data that is available. On the other hand theoretical model is the derivation from the concept of fundamental concept to understand structure. Finally structural model considers the structure and its change in kinetics. It may be 
used together with empirical data. Dispersed systems information is found from analysis of this model. Few of the functional model of different types are listed in the table below.

\begin{tabular}{|c|c|}
\hline$\sigma=\eta \dot{\gamma}$ & Newtonian model* \\
\hline$\sigma=\frac{\dot{\gamma}}{\left[\frac{1}{\eta_{0}}+K_{\mathrm{E}}(\sigma)^{\left(1 / n_{\mathrm{E}}\right)-1}\right.}$ & $\begin{array}{l}\text { Ellis model for low-shear rate data containing } \\
\qquad \eta_{0} \text { (Brodkey 1967) }\end{array}$ \\
\hline$\sigma=\left[\eta_{\infty} \dot{\gamma}+K_{\mathrm{s}} \dot{\gamma}^{n_{\mathrm{s}}}\right]$ & $\begin{array}{l}\text { Sisko model for high-shear rate data containing } \\
\eta_{\infty} \text { (Brodkey 1967) }\end{array}$ \\
\hline$\eta_{\mathrm{a}}=\eta_{\infty}+\frac{\eta_{0}-\eta_{\infty}}{1+\left(\alpha_{\mathrm{c}} \dot{\gamma}\right)^{m}}$ & $\begin{array}{l}\text { Cross model for data over a wide range of } \\
\text { shear rates* }\end{array}$ \\
\hline$\eta_{\mathrm{a}}=\eta_{\infty}+\frac{\eta_{0}-\eta_{\infty}}{\left[1+\left(\lambda_{\mathrm{c}} \dot{\gamma}\right)^{2}\right]^{N}}$ & $\begin{array}{l}\text { Carreau model for data over a wide range of } \\
\text { shear rates* }\end{array}$ \\
\hline$\sigma=\mathrm{K} \dot{\gamma}^{n}$ & $\begin{array}{l}\text { Power law model used extensively in handling } \\
\text { applications* }\end{array}$ \\
\hline$\sigma-\sigma_{0}=\eta^{\prime} \dot{\gamma}$ & Bingham model* \\
\hline$\sigma-\sigma_{0 \mathrm{H}}=K_{K} \dot{\gamma}^{n_{\mathrm{H}}}$ & Herschel-Bulkley model* \\
\hline$\sigma^{0.5}=K_{0 \mathrm{c}}+K_{\mathrm{c}}(\dot{\gamma})^{0.5}$ & $\begin{array}{l}\text { Casson model used especially in treating data } \\
\text { on chocolates* }\end{array}$ \\
\hline$\sigma^{0.5}-\sigma_{0 \mathrm{M}}=K_{\mathrm{M}} \dot{\gamma}^{n_{\mathrm{M}}}$ & $\begin{array}{l}\text { Mizrahi and Berk (1972) model is a modifica- } \\
\text { tion of the Casson model }\end{array}$ \\
\hline$\sigma^{n_{1}}=\sigma_{0}^{n_{1}}+\eta_{\infty}(\dot{\gamma})^{n_{2}}$ & Generalized model of Ofoli et al. (1987)* \\
\hline$\sigma=\left[\left(\sigma_{o \mathrm{~V}}\right)^{1 / n_{\mathrm{V}}}+K_{\mathrm{V} \dot{\gamma}}\right]^{n_{\mathrm{V}}}$ & $\begin{array}{l}\text { Vocadlo (Vocadlo and Moo Young 1969) } \\
\text { model }\end{array}$ \\
\hline
\end{tabular}

Table 3.1: Flow models for describing shear stress vs strain rate data [80]

Among these flow models we have used Herschel-Bulkley model [81] for shear stress and strain rate result analysis. We have also used power law model for total pressure with respect to packing density. Details about these two models are described in respective result section.

Shear actually means shear strain that is the deformation of a material when its parallel internal surfaces slides past each other. Now this shear strain produces shear stress and from the ratio of shear stress and shear strain rate we could achieve our desired shear viscosity. Now the shear strain could be applied in two ways (a) simple shear and (b) pure shear [82]. Simple shear is the most general shear strain of materials. In this strain the force is acted parallel to the surface 
that causes the surface closer to the applied force move far than the surface away from the force that cause a change in the materials shape. There is no compression or extension occurs but rotation occurs that cause the change in shape but not volume. On the other hand if the force is acted perpendicular to the surface that cause the surface perpendicular to the applied forces direction to compress and simultaneously the opposite surfaces to extend then it is called pure shear. There is no rotation in this type of shear occurs and therefore orthogonal planes remains orthogonal. In this case the shape does not change but volume does change. This two type of shear also could take place simultaneously.

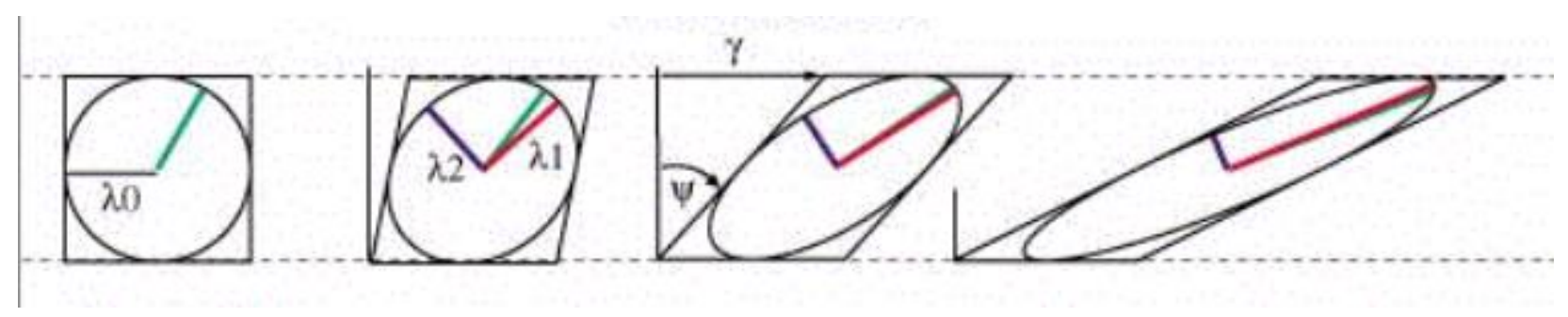

(a)

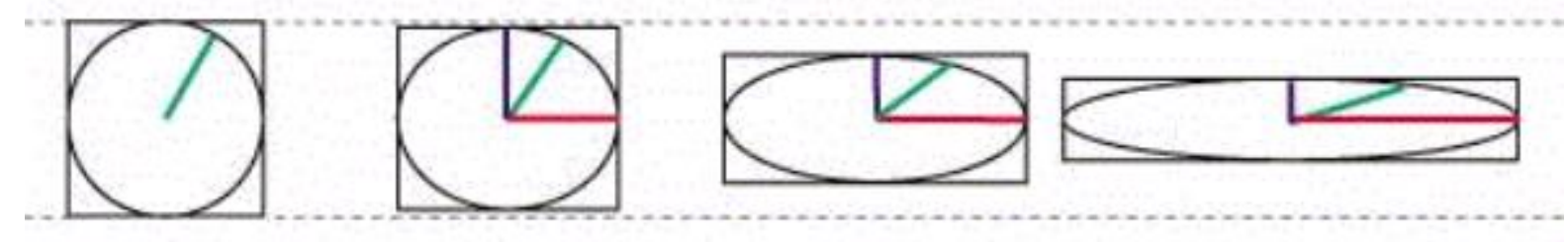

(b)

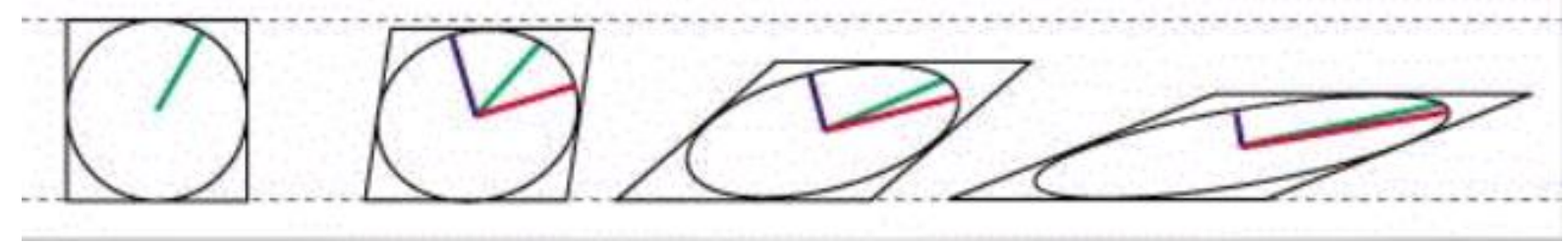

(c)

Figure 3.8: A square shaped object under (a) simple shear (b) pure shear and (c) simultaneous simple and pure shear [83]

In our Non-equilibrium molecular dynamics simulation for continuous strained system we have used pure shear strain to change the area of our 2 dimensional simulation box during running the dynamics with different rates equals to $0.001,0.002,0.003,0.004,0.005,0.006,0.007$, 
$0.008,0.009,0.01,0.02,0.03,0.04,0.05,0.06,0.07,0.08,0.09$. We have compressed the simulation box in $\mathrm{y}$ direction and let it expand in $\mathrm{x}$ direction with same proportion. We could have change the box dimension non-linearly with time while keeping the strain rate constant. But that would not be ideal for the output data processing. We have used constant engineering strain rate that change the box dimension linearly with time. If $L_{0}$ is the initial box length and erate is engineering strain rate then after time dt the box length $\mathrm{L}$ will be,

$$
L=L_{0}(1+\text { erate } * \mathrm{dt})
$$

We have remapped the fluids of the simulation box in order to match with the change of the box. Finally we have not kept any dimensions of the simulation box shrink-wrapped.

\section{CHAPTER-4 \\ RESULTS}

\subsection{Shear Stress and Viscosity vs Strain Rate}

We have three different kind of star particles (a) $3 \mathrm{leg}$ (b) 5 leg and (c) 7 leg where each of them has various number of leg lengths . For 3 leg star particles the leg lengths (L) are 0.0, 0.5, $1.0,1.5,2.0,2.5,3.0,3.5,4.0,4.5,5.0,5.5,6.0$. For 5 leg star particles the leg lengths (L) are $0.0,0.5,1.0,1.2,1.3,1.5,1.6,2.0,2.5,3.0$ and for 7 leg star particles the leg lengths (L) are $0.0,0.5,1.0,1.3,1.5,1.6,1.7,1.8,2.0,2.5$. Each leg lengths has 30 different configurations where each configuration has different initial condition. We have infused each configuration of star particles into SRD particle which have fluid like properties separately and run our simulation after removing the overlapping of stars until the simulation reaches in equilibrium state. Then we applied pure shear strain along Y directions and allow the system to expand orthogonally in the $\mathrm{X}$ direction. We have used strain rate equals $0.001,0.002,0.003,0.004$, $0.005,0.006,0.007,0.008,0.009,0.01,0.02,0.03,0.04,0.05,0.06,0.07,0.08,0.09$ for each system. 
From the shear stress vs strain rate plot in Figure 4.1(a), 4.2(a) and 4.3(a) we have found that for lower strain rate we get lower shear stress and for a gradual increase in strain rate the shear stress is also increased exponentially. The packing fraction of each system also plays an important role in shear stress value. For same strain rate the system with higher packing fraction gives higher stress because of jamming. For lower strain rate and very low packing fraction the system gives the lowest stress values and therefore it might reach in negative stress also. Again the exponential increment of stress with respect to strain rate could be estimated using the Herschel-Bulkley model [80]. This model includes the yield stress into the power law model. The model shows the relationship with respect to yield stress, slope and power as following,

$$
\sigma=A \dot{\gamma}^{\alpha}+\sigma_{y}
$$

Here, $A$ is the slope, $\alpha$ is the exponent and $\sigma_{y}$ is the yield shear stress. We have plotted the nonlinear regression plotting of shear stress vs strain rate using Herschel-Bulkley equation. The fitting used least square method [84]. These fitting plots for shear stress with respect to strain rate are showed in Figure 4.1 (b), 4.2(b) and 4.3(b). Also to have a clear understanding how the yield stress and exponent varies with respect to strain rate we have plotted exponent vs packing fraction and yield stress vs packing fraction plots by considering different strain rates. This plotting of Figure 4.1(c), 4.2(c) and 4.3(c) proves that only for 3 leg star particles with very lower packing fraction the exponent reaches less than 1 which means the shear thinning behavior of the fluid. But except that every other packing densities for $3 \mathrm{leg}, 5 \mathrm{leg}$ and $7 \mathrm{leg}$ packings the exponent is higher than 1 which means the shear thickening behavior of fluid. This implies that the overall system is mostly behaving as shear thickening fluid for both higher and lower packing fraction. Also the yield stresses that plotted alongside the exponents. For low packing densities plotting in the figures demonstrates that the yield stresses are also low and with the increase in packing density the yield stresses also increases exponentially. It indicates the shifting from liquid-like state to a solid-like state of the system happened due to the jamming transition. 
(a)

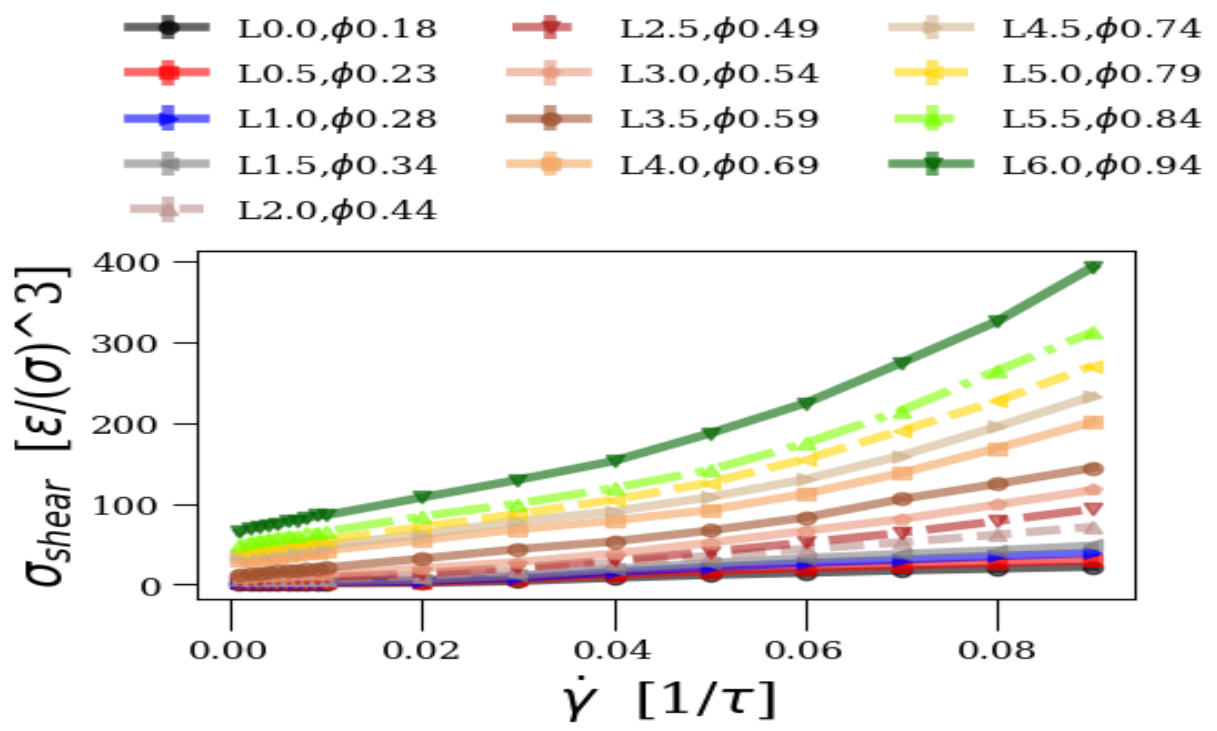

(b)
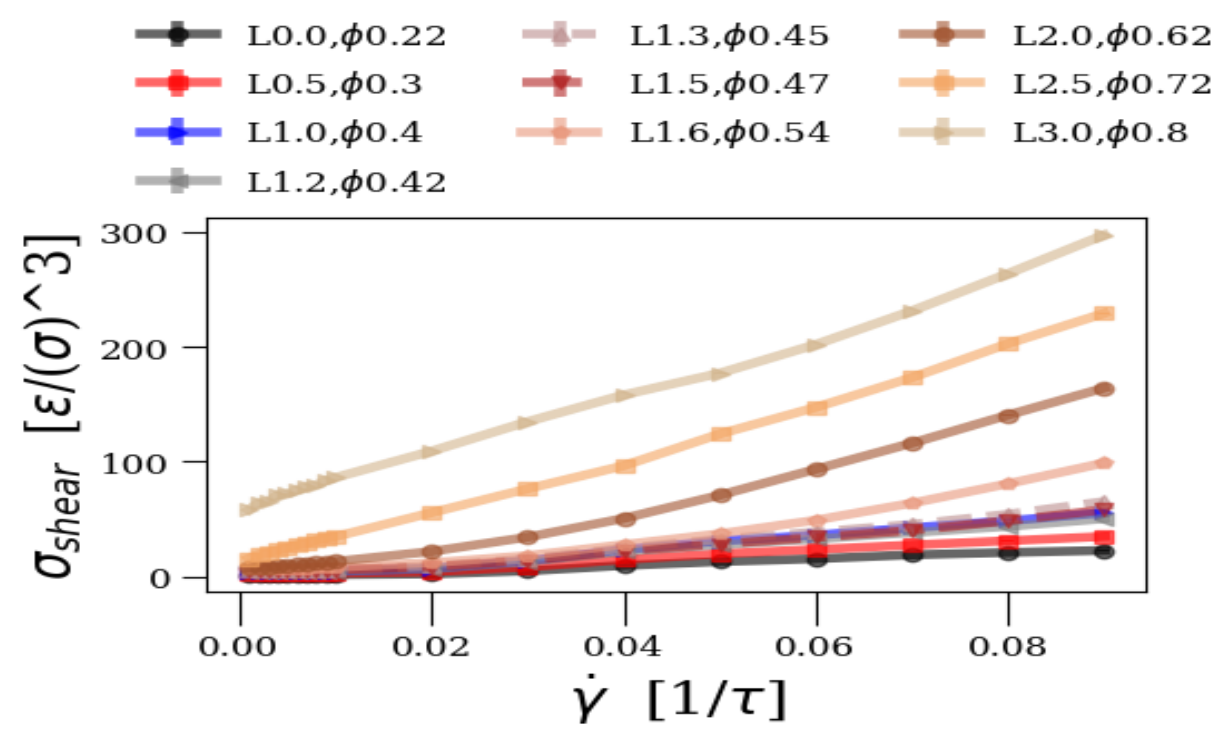

(c)
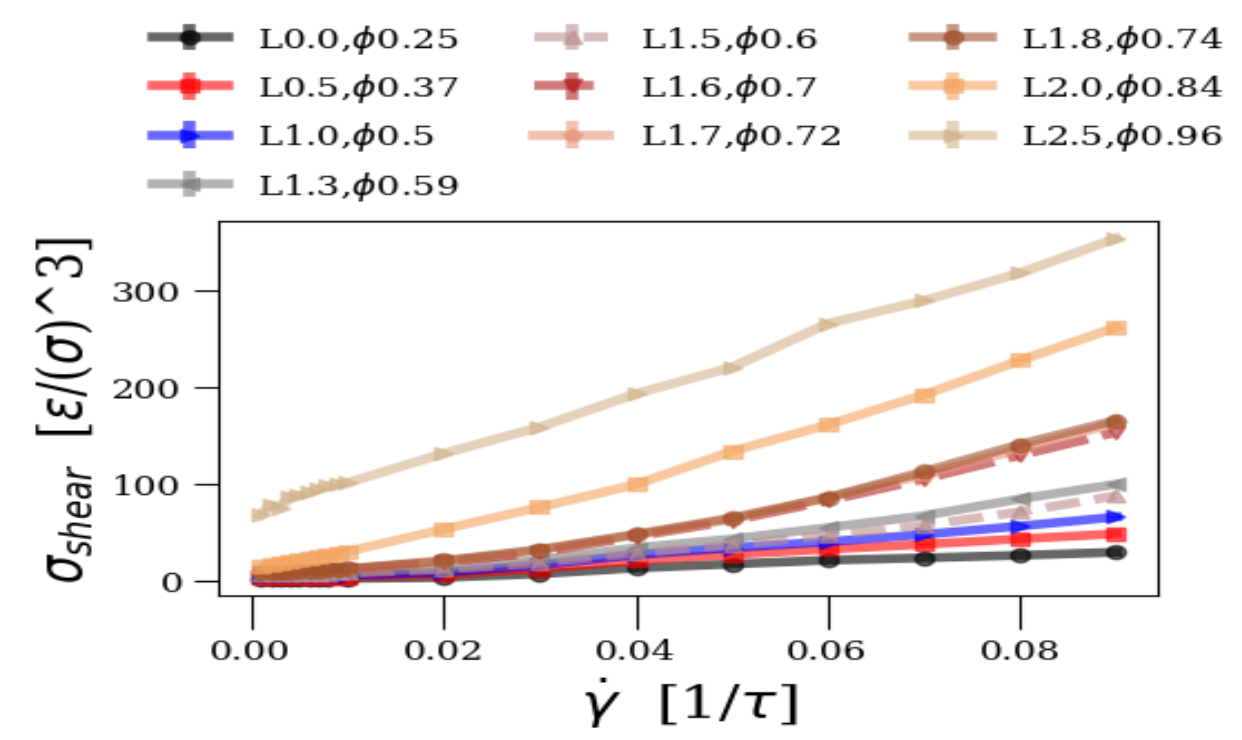

Figure 4.1: Shear stress vs shear strain rate plotting for (a)3 leg (b)5 leg and (c)7 leg star particles. 

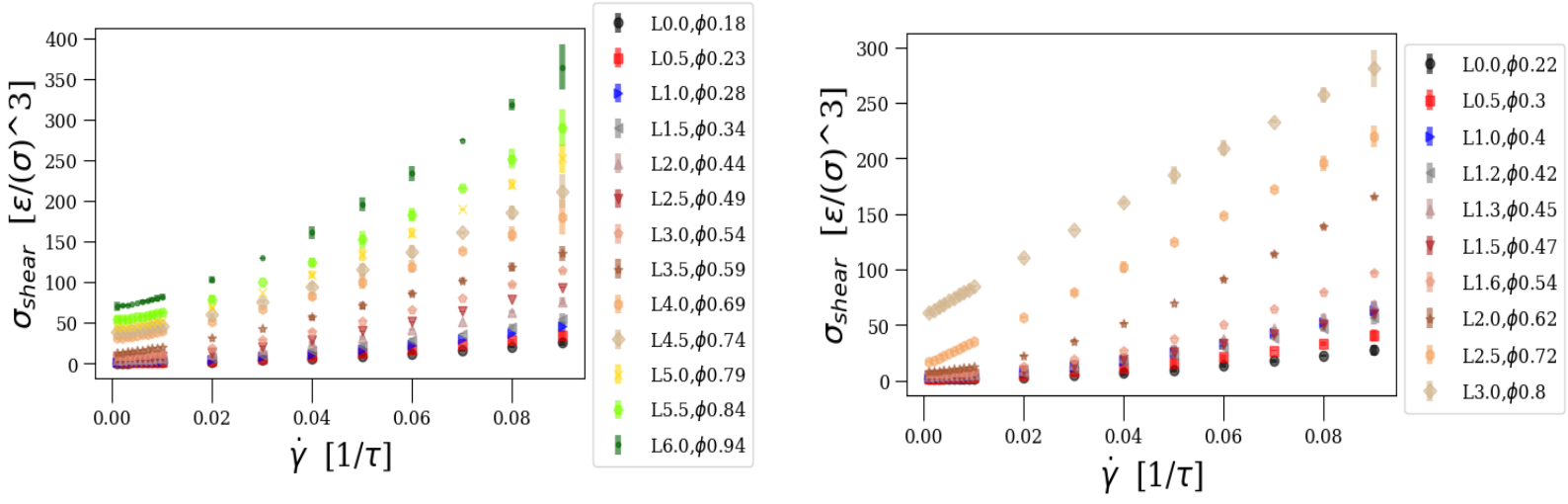

(a)

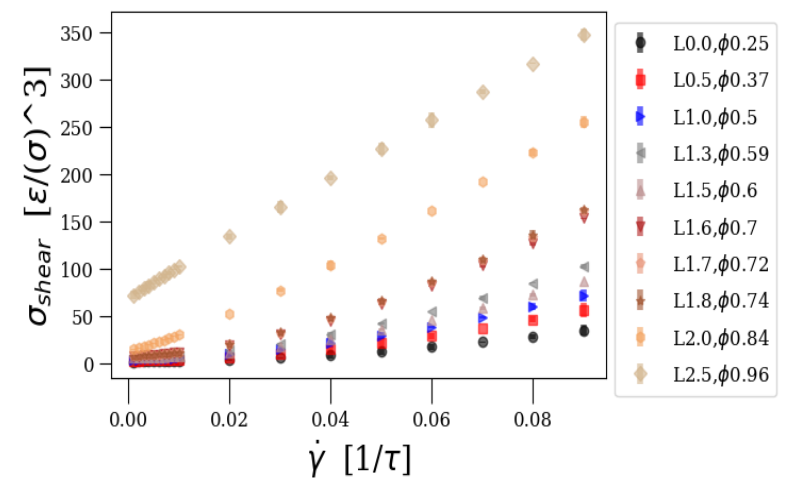

(b)

(c)

Figure 4.2: Fitting in Herschel-bulkley model for for (a) 3 leg (b) 5 leg and (c) 7 leg star particles.
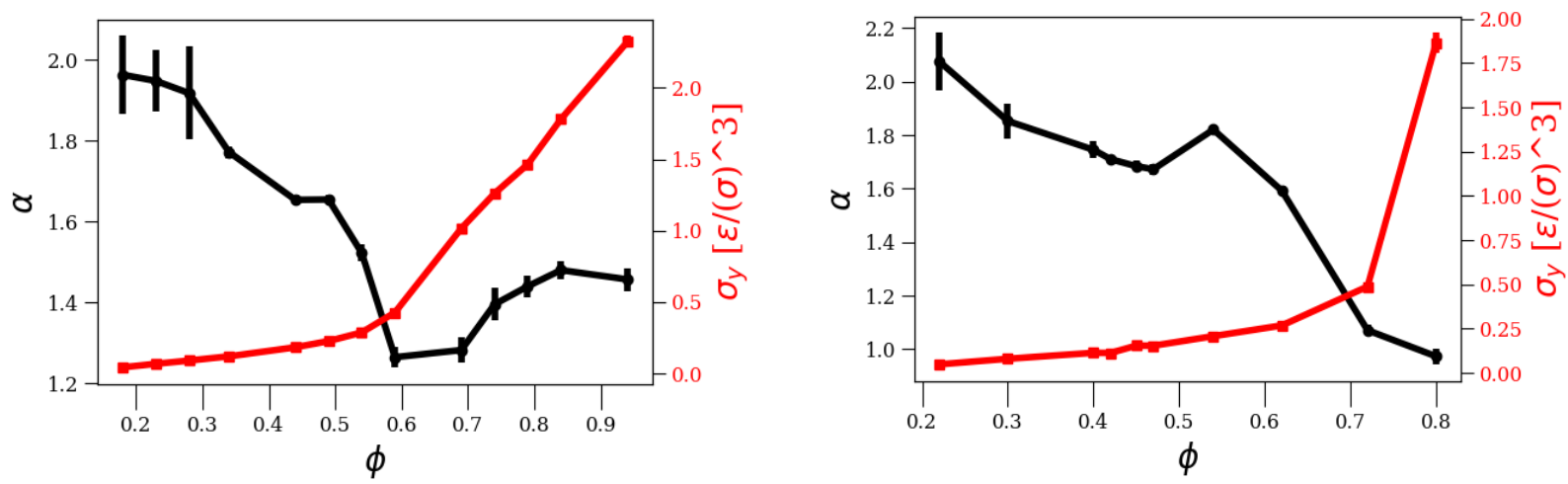

(a)

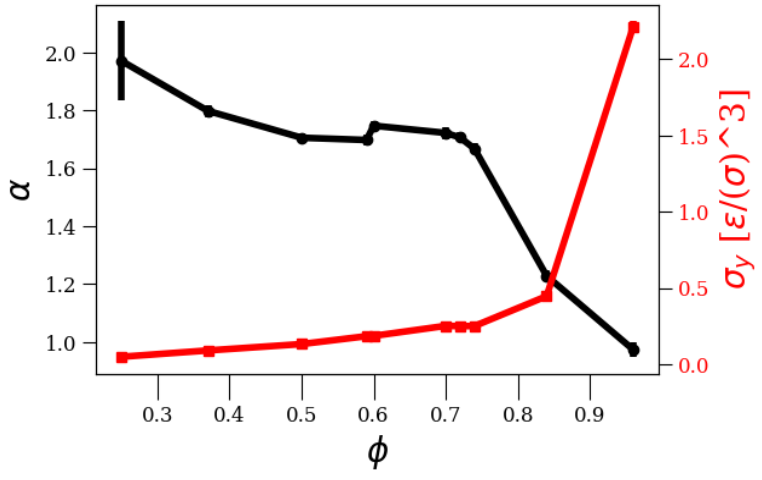

(b)

(c)

Figure 4.3: Exponent and yield stress plotting with respect to packing fraction for (a) 3 leg (b) 5 leg and (c) 7 leg star particles. 
We can also conclude about the shear thickening behavior based on the viscosity vs strain rate plotting. From definition of shear thinning we know that the viscosity decreases with the rate of applied shear strain. Again for shear thickening the viscosity increases with the rate of shear strain. Modern paints, ketchup quicksand, toothpaste etc. are example of shear thinning fluid. On the other hand, corn stark dissolved in water is an example of shear thickening fluid. It is clearly visible from the Figure $4.4(\mathrm{a}, \mathrm{b}, \mathrm{c})$ that for the increase in shear rate the viscosity also increases. Although for higher packing the increment is higher we can safely conclude that viscosity is increasing for the increment of strain rate and that is why our fluid with infused star particles with different leg lengths and number of legs in general shows shear thickening behavior.

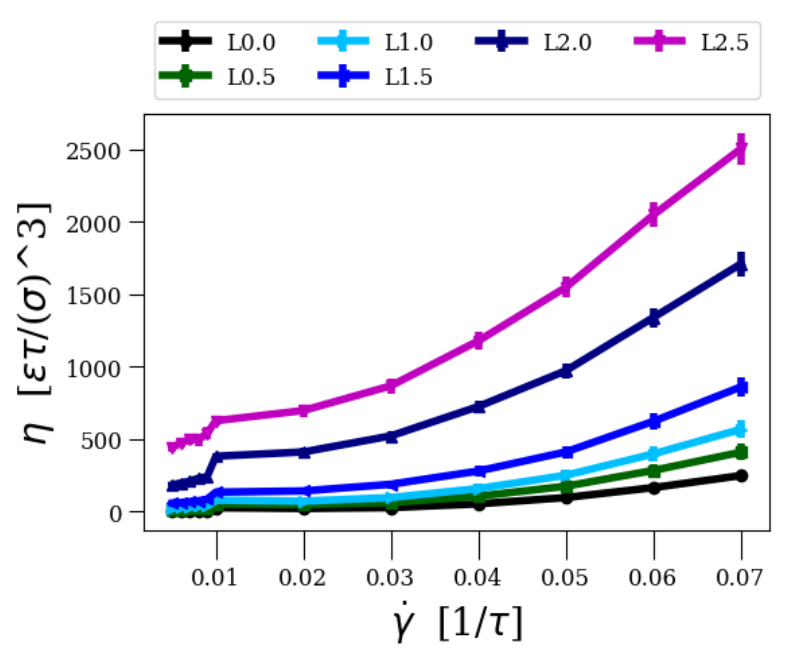

(a)

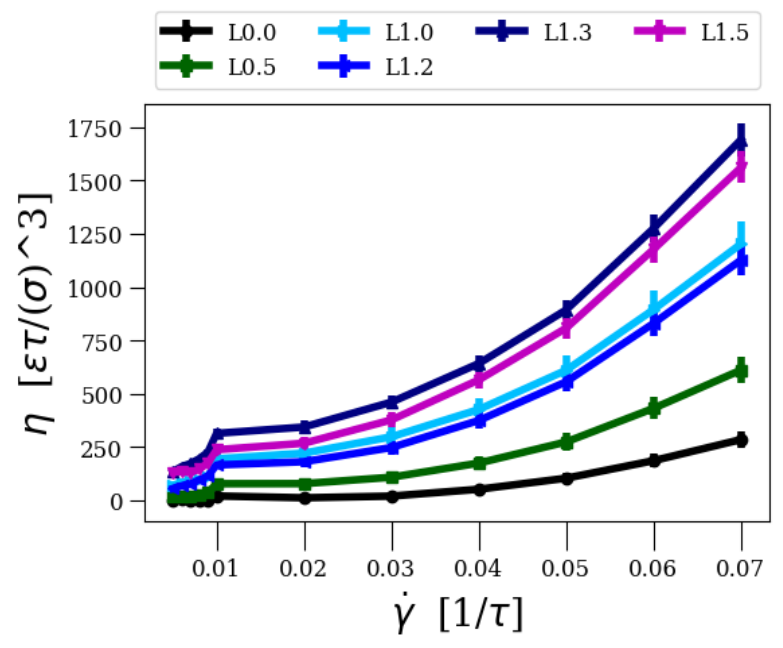

(b)

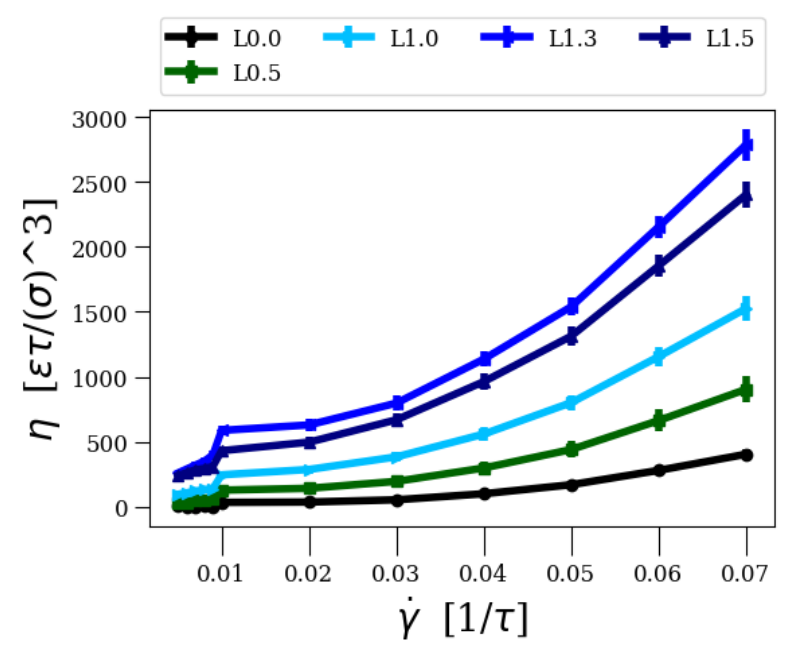

(c)

Figure 4.4: Viscosity plotting with respect to strain rate of (a) 3 leg (b) 5 leg and (c) 7 leg star particles for different packing fraction. 


\subsection{Pressure and jamming}

The total pressure calculated for systems under shear could also be used to understand the jamming transition more clearly. We have calculated the steady state average total pressure for different systems with different packing density under multiple constant shear rate applied for sufficiently longer time period. In principle we expect to see the total pressure vanishes for packing fraction lower than jamming and rise to finite values after jamming packing fraction [17], [85]. From Figure 4.5 (a, b, c) we have found that after jamming point the total pressure values reaches finite values by diverging from that point. At higher packing fraction, the higher strain rate cause the total pressure diverge further away than lower strain rate. Using these plotting we have found the jamming packing fraction for systems consists with 3 leg, 5 leg and 7 leg star particles.

We have also used the power law model [80] to show the relationship of pressure and packing fraction after the jamming transition.

$$
P= \begin{cases}B\left(\varphi-\varphi_{c}\right)^{\beta} & , \text { when } \varphi>\varphi_{c} \\ 0 & , \text { when } \varphi \leq \varphi_{c}\end{cases}
$$

Here, $\mathrm{B}$ is the slope, $\beta$ is the exponent and $\varphi_{c}$ is the jamming packing fraction. In the Figure $4.6(a, b, c)$ we have showed how the exponent and jamming packing fraction varies with respect to the applied strain rate into different system that consists infused 3leg, 5leg and 7leg star particles with different packing density. 


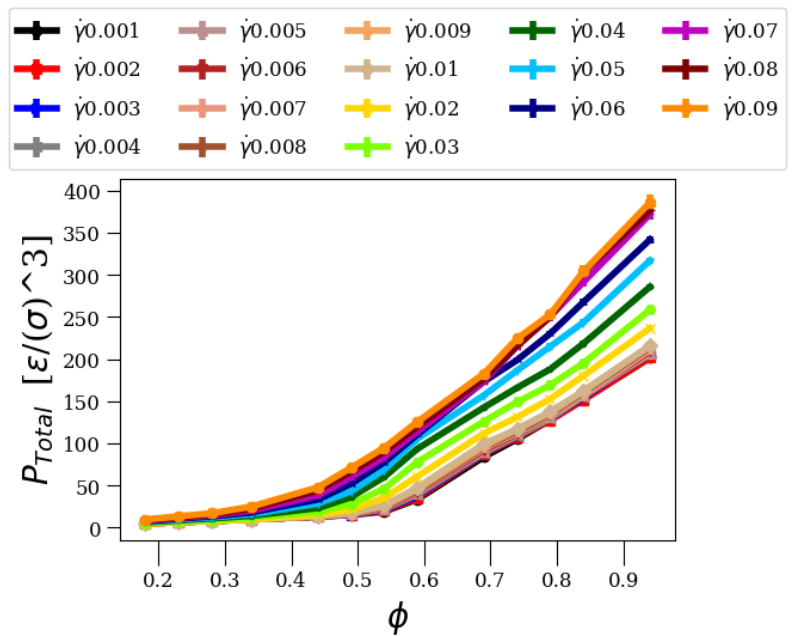

(a)
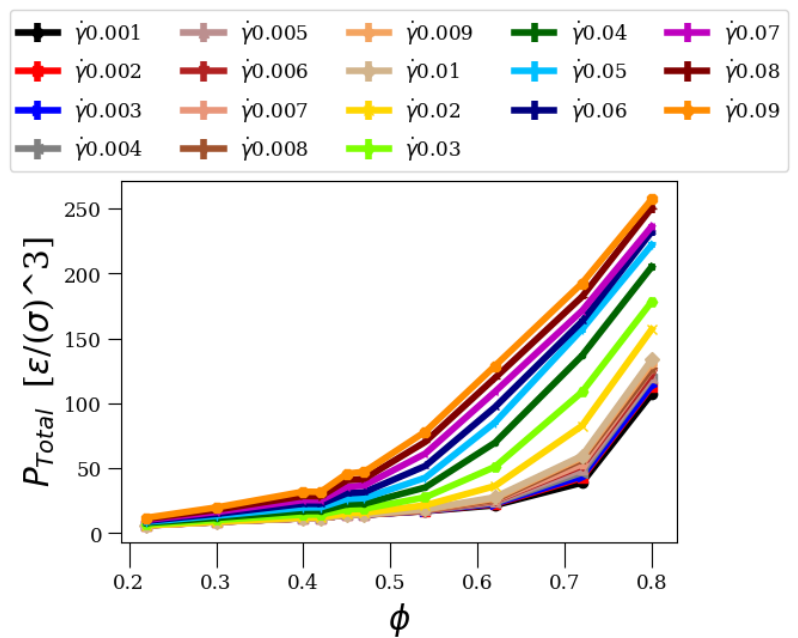

(b)

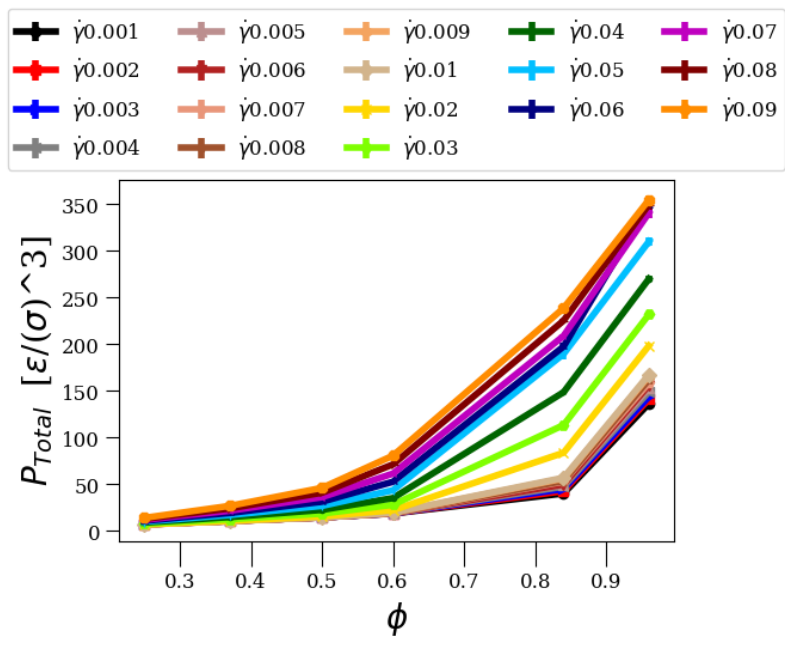

(c)

Figure 4.5: Total pressure plotting with respect to packing fraction for (a) 3 leg (b) 5 leg and (c) 7 leg star particles for strain rate 0.001, 0.002, 0.003, 0.004, 0.005, 0.006, 0.007, 0.008, $0.009,0.01,0.02,0.03,0.04,0.05,0.06,0.07,0.08,0.09$. 


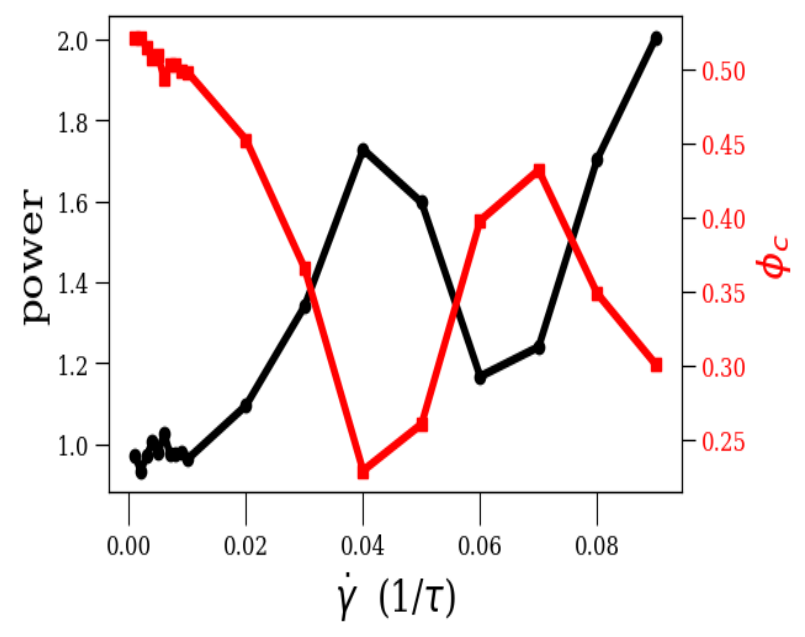

(a)

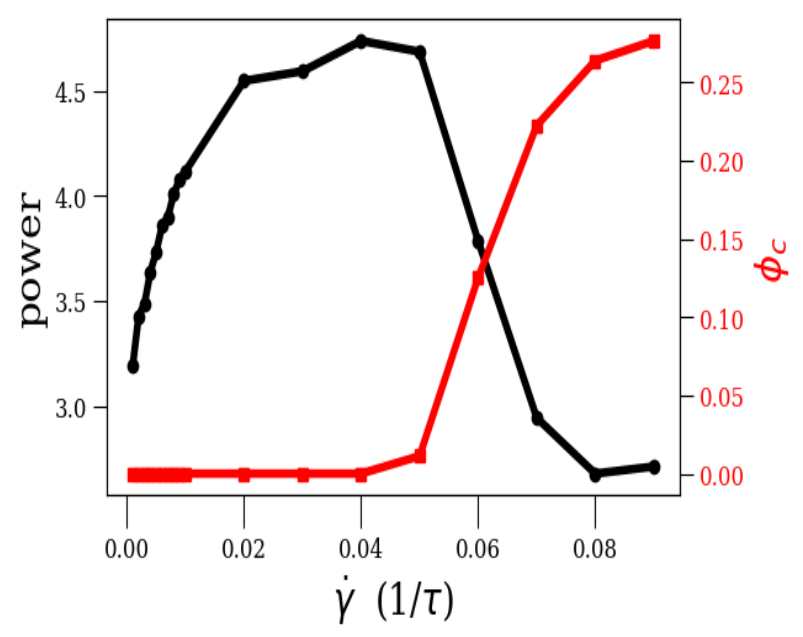

(b)

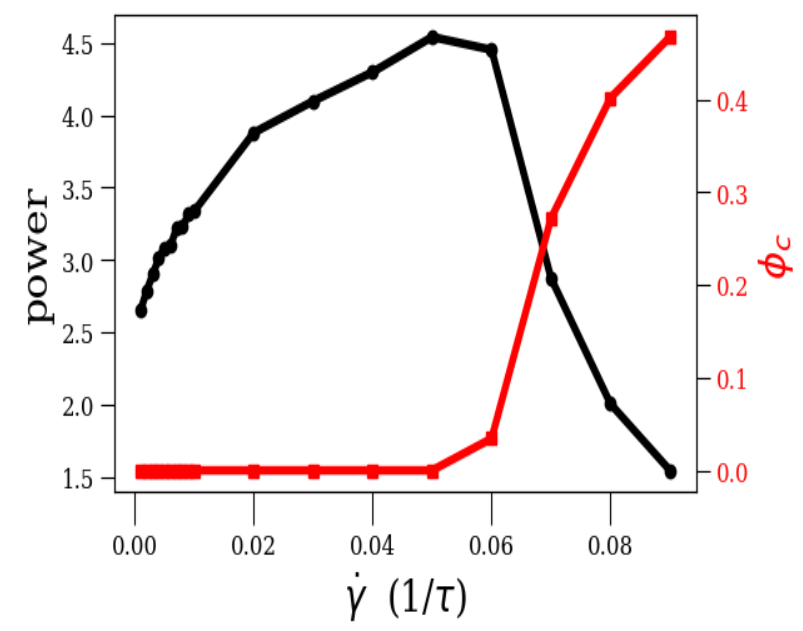

(c)

Figure 4.6: Error bar plotting of exponent and jamming packing fraction with respect to strain rate for (a) 3 leg (b) 5 leg and (c) 7 leg star particles.

\subsection{Jammed System Under Uniform Shear Strain Rate}

We are considering shear driven jamming under different shear strain rate. For each configuration of system we applied strain rate equals $0.001,0.002,0.003,0.004,0.005,0.006$, $0.007,0.008,0.009,0.01,0.02,0.03,0.04,0.05,0.06,0.07,0.08,0.09$. We dumped the strain values, total pressure values and shear stress values for each strain rate. Then for each strain rate we took the average total pressure and average shear stress for each strain window $\Delta \Upsilon=1000$. The error bar is the standard error of mean which is calculated from the 
configurations with equal leg length and number of legs. The system was expected to lose memory of its initial configuration when sheared for long time [17]. At the same time for higher packing fraction and lower strain rates the initial configuration memory persists for longer strains than lower packing fraction or higher strain rates. We have plotted average pressure and average shear stress for lower strain rate 0.001 and higher strain rate 0.009 . For different packing densities, it is found that the different packings and strain rate follows the same trend of coming into the steady state we were expecting for all $3 \mathrm{leg}, 5 \mathrm{leg}$ and $7 \mathrm{leg}$ star particles.

(a)

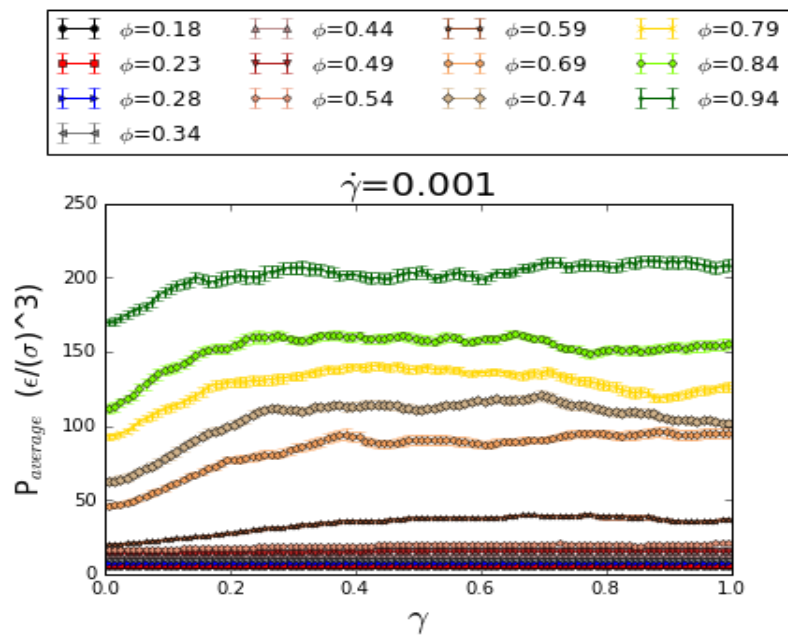

(c)

\begin{tabular}{|c|c|c|c|c|c|c|c|}
\hline$\Phi \Phi$ & $\phi=0.18$ & $\Phi$ & $\phi=0.44$ & $\Phi \mp$ & $\phi=0.59$ & 11 & $\phi=0.79$ \\
\hline$\Phi$ & $\phi=0.23$ & \pm & $\phi=0.49$ & $\phi$ & $\phi=0.69$ & क & $\phi=0.84$ \\
\hline \pm & $\phi=0.28$ & $\Phi \Phi$ & $\phi=0.54$ & $\phi$ & $\phi=0.74$ & $I I$ & $\phi=0.94$ \\
\hline \pm & $\phi=0.34$ & & & & & & \\
\hline
\end{tabular}

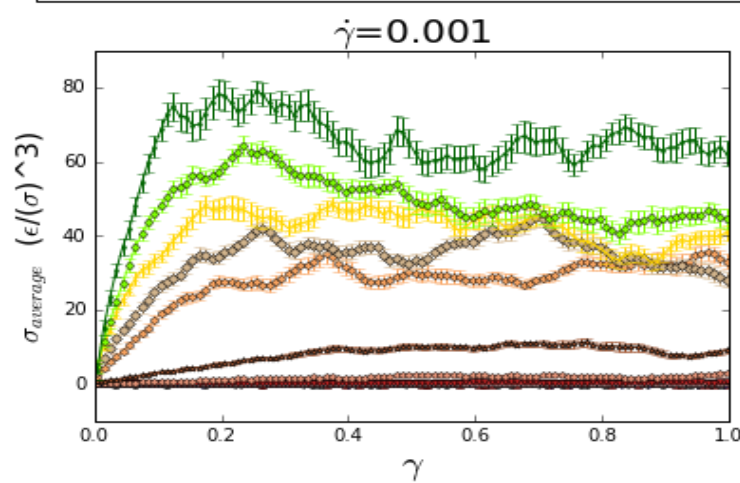

(b)

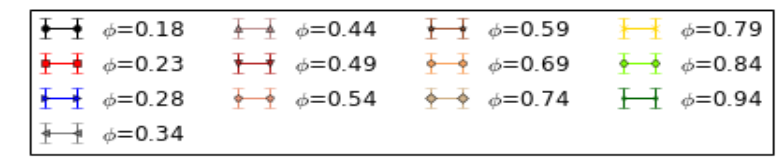

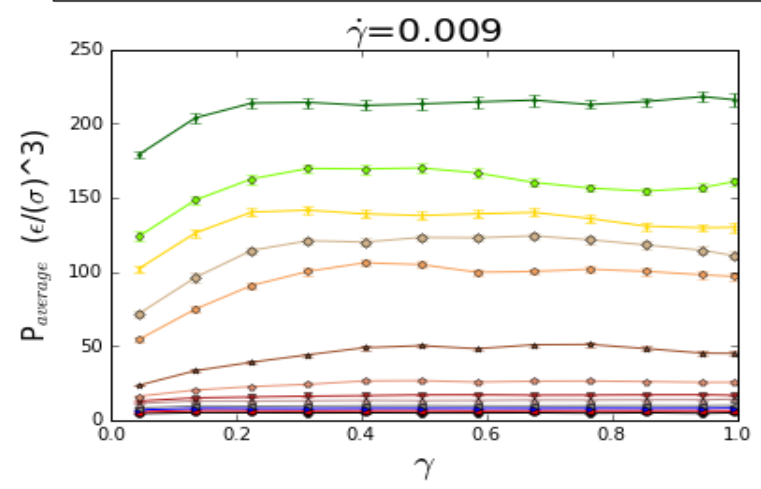

(d)

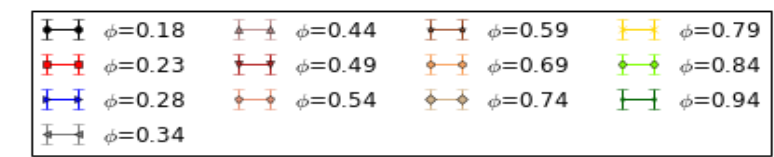

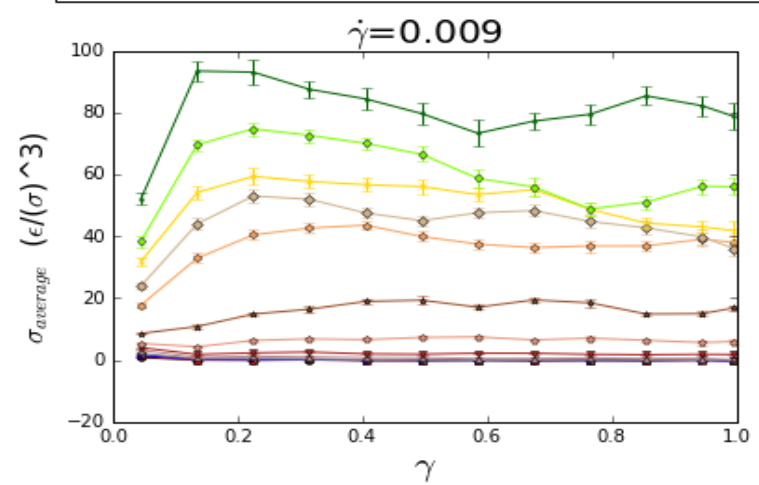

Figure 4.7: Total Pressure vs strain plot for low strain rate (a) 0.001 and high strain rate (b) 0.009 of 3 leg star particles for packing fraction $0.18,0.23,0.28,0.34,0.44,0.49,0.54,0.59$, $0.69,0.74,0.79,0.84,0.94$. Average shear stress with respect to strain is also plotted for strain rate (c) 0.001 and (d) 0.009 of 3 leg star particles for same packing density. 
(a)

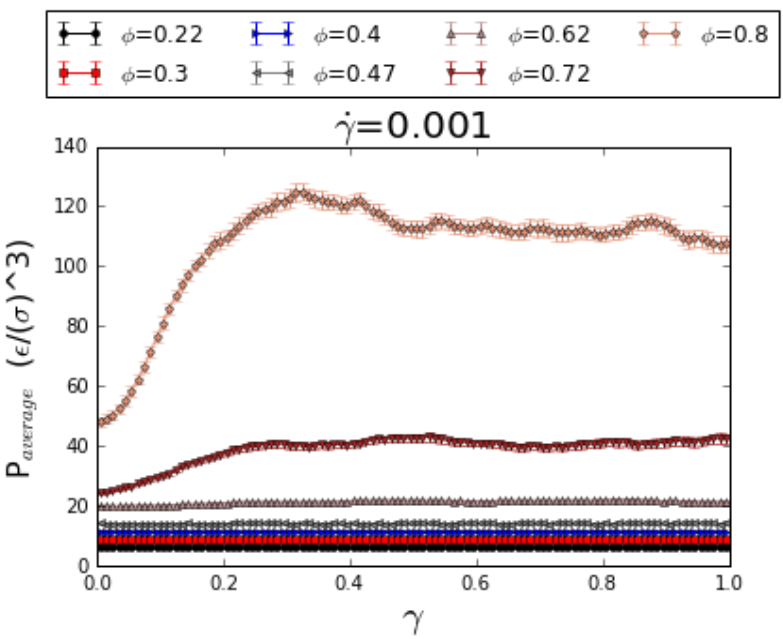

(c)

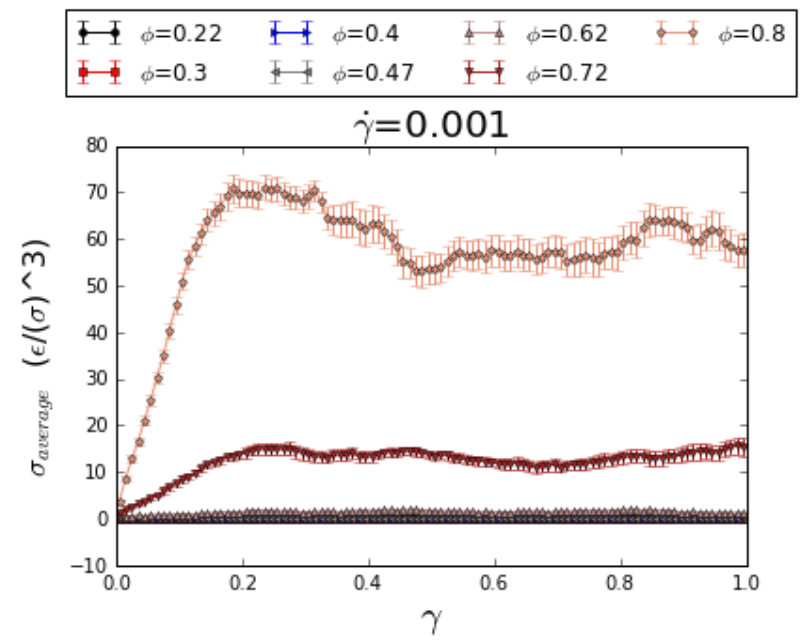

(b)

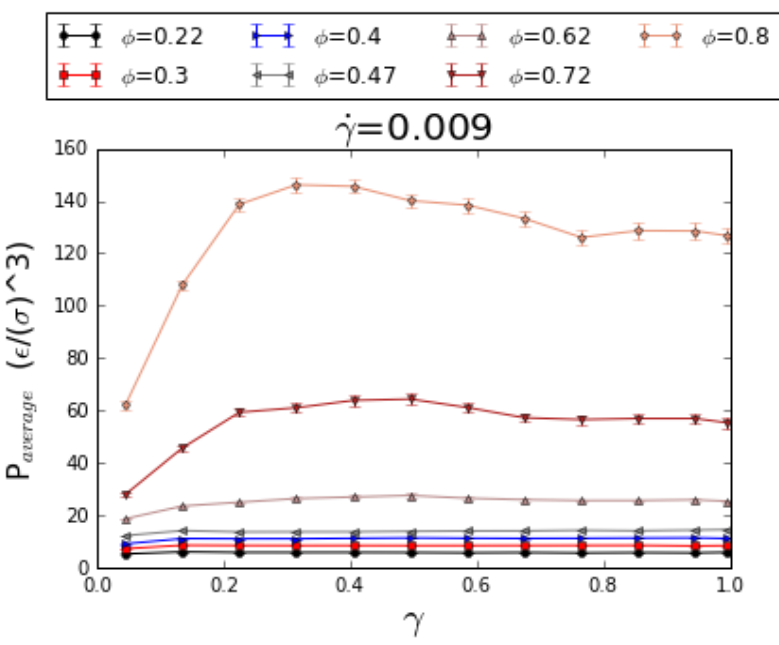

(d)

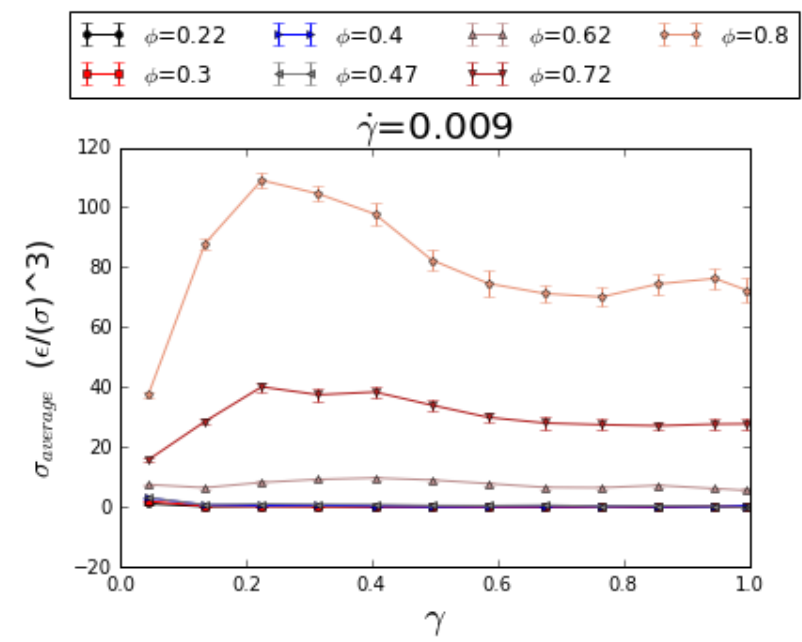

Figure 4.8: Total Pressure vs strain plot for low strain rate (a) 0.001 and high strain rate (b) 0.009 of 5 leg star particles for packing fraction $0.22,0.30,0.40,0.47,0.62,0.72,0.80$. Average shear stress with respect to strain is also plotted for strain rate (c) 0.001 and (d) 0.009 of 5 leg star particles for same packing density. 


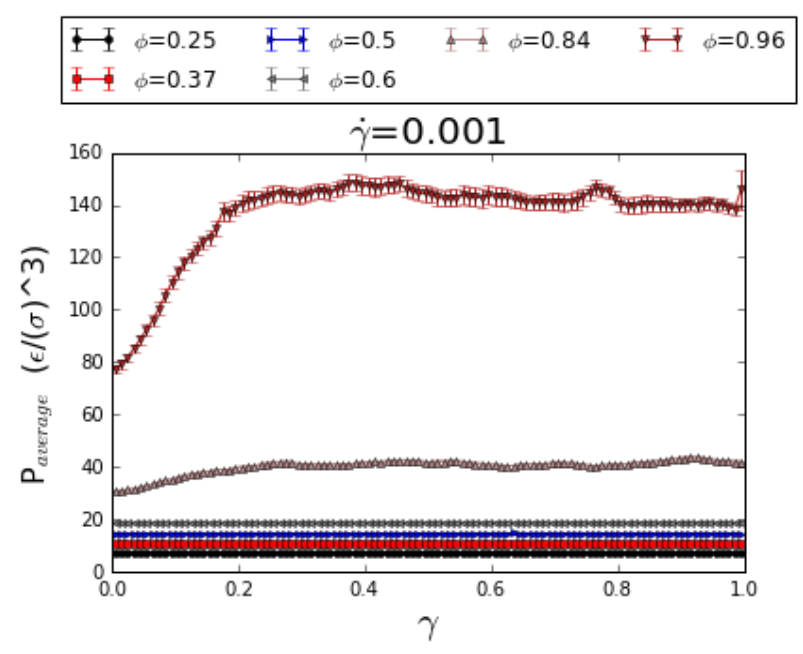

(c)

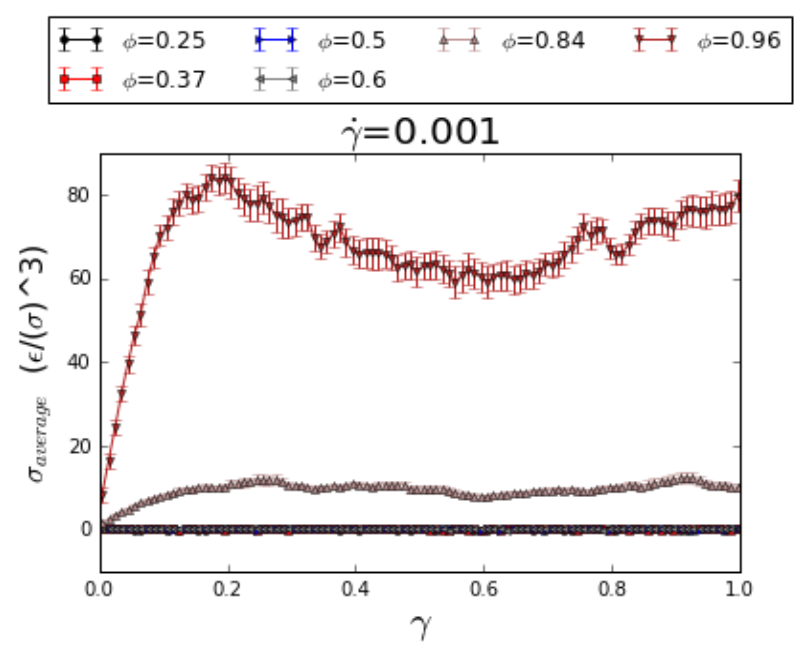

(b)

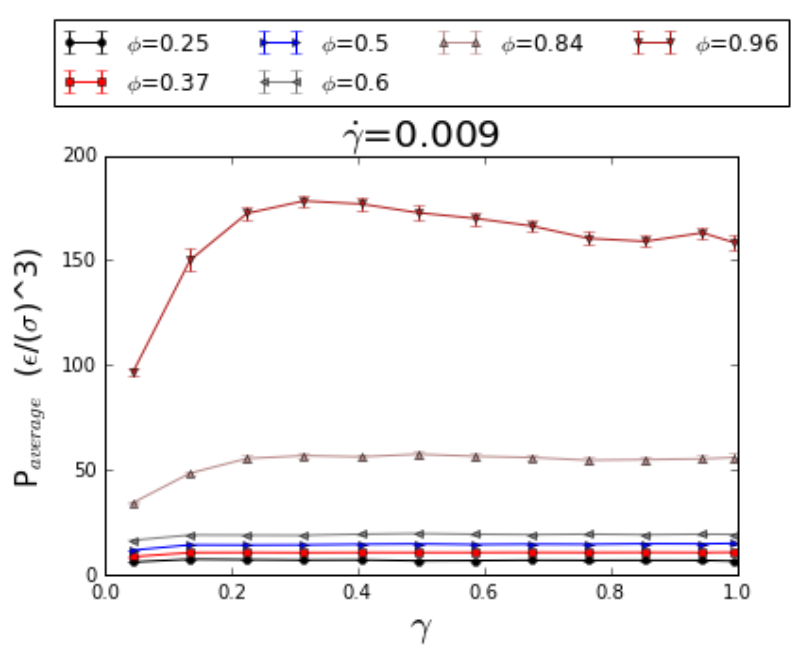

(d)

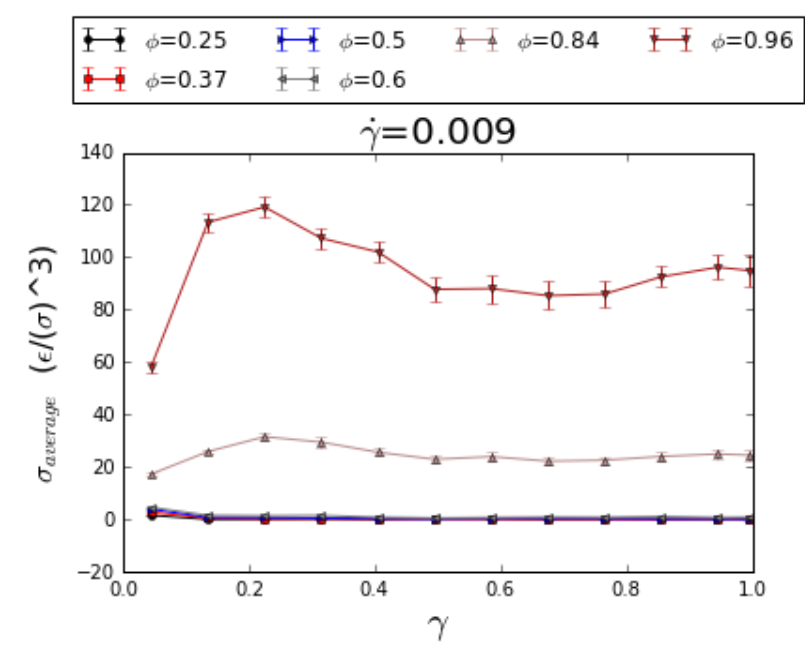

Figure 4.9: Total Pressure vs strain plot for low strain rate (a) 0.001 and high strain rate (b) 0.009 of 7 leg star particles for packing fraction $0.25,0.37,0.50,0.60,0.84,0.96$. Average shear stress with respect to strain is also plotted for strain rate (c) 0.001 and (d) 0.009 of 7 leg star particles for same packing density. 


\subsection{Viscosity}

Controlling the viscosity of the fluid by infusing star particles with variable number of legs and leg lengths was the prime focus of our work. We have calculated the shear stress from the simulation and dumped the stress values along with the strain values. Then we calculate the average shear stress for that specific strain rate. By this way we create arrays of shear stress vs strain rates for each configurations of leg lengths for specific number of legs. Then we have calculated the viscosity by calculating the slope from shear stress and strain rate plotting for each configurations. These viscosity values are again kept as an array of viscosity, leg length and packing fraction. As we have multiple viscosity values from same configuration we take the mean viscosity value for each configuration and for error bar plotting we use the Standard error of mean formula. Finally we plot the viscosity vs Leg length plot and viscosity vs packing fraction plot to see how the viscosity changes depending on these parameters.

The plotting for viscosity with respect to leg length (L) and packing fraction $(\varphi)$ is showed in Figure $4.10(a, b)$ respectively. The red line of these plots are the viscosity plot for 3 leg star particles. It shows clearly that the viscosity was almost close to zero initially when the fluid has no particles or particles with very small leg length that generates very small packing density. But as soon as the leg lengths increased the viscosity value starts to rise and for leg length $\mathrm{L}=2.5$ or $\varphi \approx 0.5$ the viscosity jumps to a higher value and it continues as the leg lengths continues to increase. Finally the viscosity reaches almost close to 15000 for $\mathrm{L}=6.0$ or $\varphi=0.94$ . Similarly for 5 leg and 7 leg star particle infusion the viscosity value indicated as green and blue line respectively jumps at $\mathrm{L}=1.5$ or $\varphi \approx 0.5$ and reaches almost same viscosity value as the 3 leg particles. Also from the error bar it is clear that at higher leg lengths the variation of viscosity is also higher. 
(a)

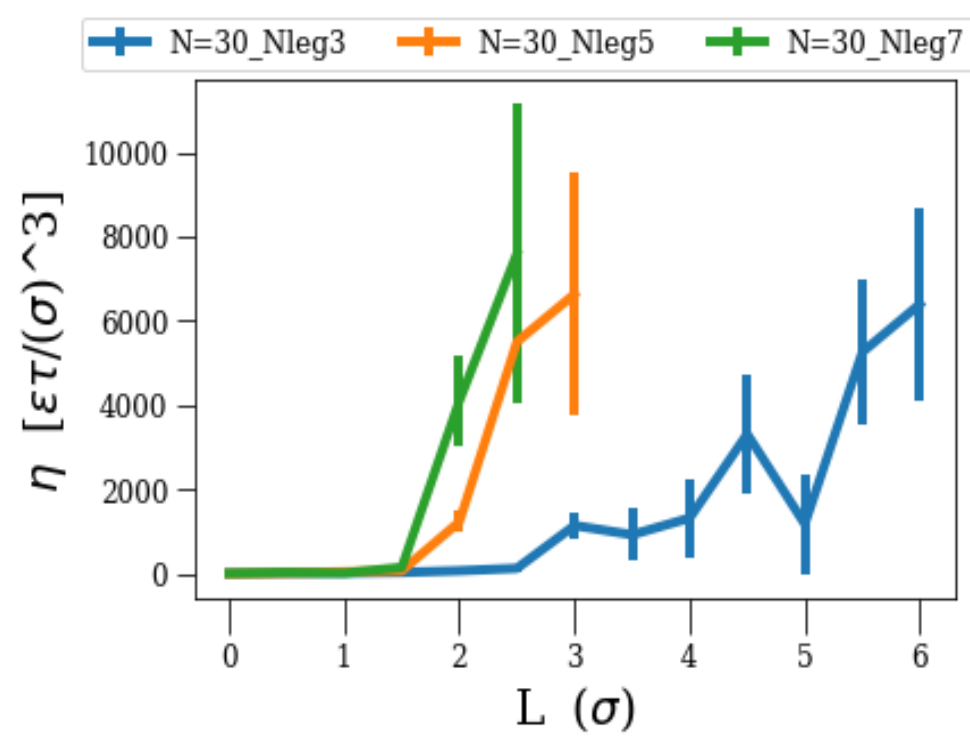

(b)

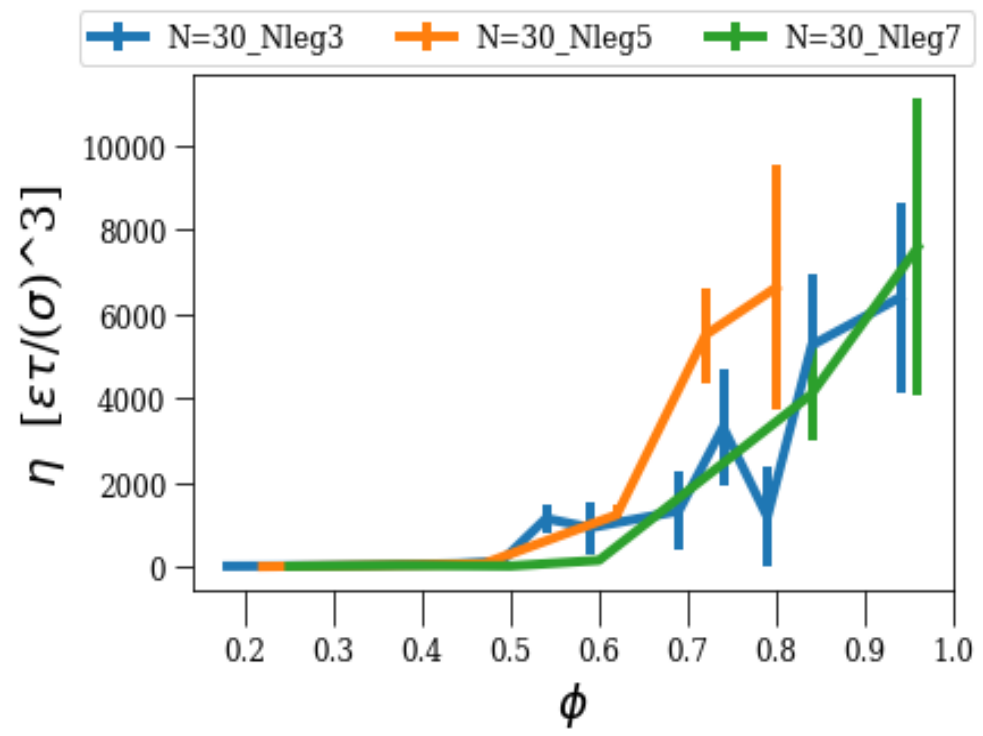

Figure 4.10: Viscosity vs (a) Leg length and (b) packing fraction plotting for 3 leg, 5 leg and 7 leg star particles.

\subsection{Diffusivity}

We have infused the star particles inside a square shaped (30*30 square unit) simulation box filled with fluid like SRD particles. For same number of leg we have calculated the diffusivity of both the Big star particles and small SRD fluids and plotted with respect to leg length (L). The diffusivity is calculated by measuring the slope of mean square displacement with respect 
to time. The higher the leg length of star particles the lower the diffusion of both star particles and fluids. As we discussed earlier the diffusivity depends on mean square displacements of the particles. The longer leg prevents the fluids as well as themselves from one place to another and hence the diffusivity falls. This kind of behavior is identical for 3 leg , 5 leg and 7 leg star particles.

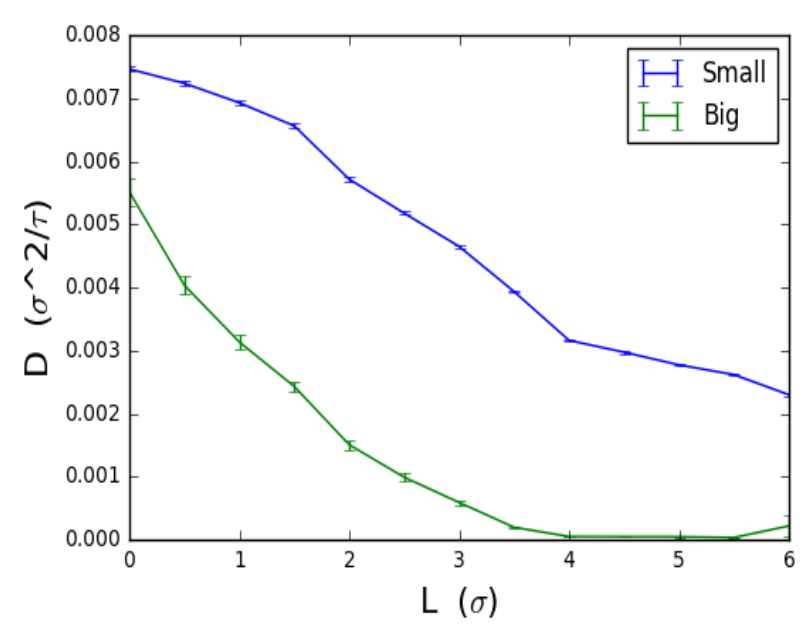

(a'

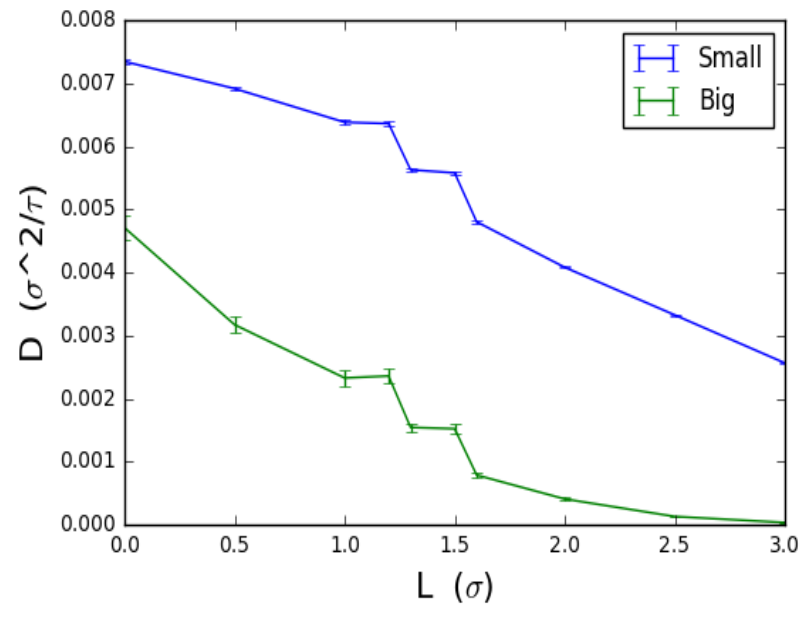

(b)

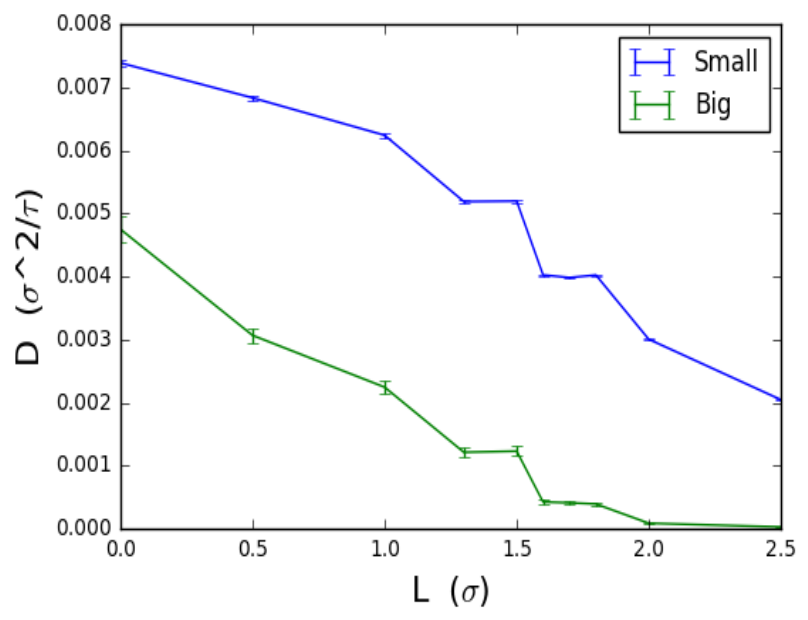

(c)

Figure 4.11: Diffusivity vs Leg length plotting of small SRD particles and big star particles for (a) 3 leg (b) 5 leg and (c) 7 leg star particles. 


\subsection{Radial Distribution Function (RDF)}

The radial distribution function $\mathrm{g}(\mathrm{r})$ otherwise known as pair correlation function gives the idea about the structure of finding the next star particles center of mass from a reference star particles center of mass. We have taken each $3 \mathrm{leg}$, 5leg and 7 leg star particles with all of their leg lengths, calculated their radial distribution function and finally plotted with the maximum radial distance $\mathrm{r}$. These plotting of radial distribution function for 3 leg, 5 leg and 7 leg star particles with various leg lengths (L) and packing fraction $(\varphi)$ are showed in Figure 4.12 .

We have found that for each of the plotting has zero $g(r)$ value at initial radial distance. The reason is at the very beginning of radial increment there is no other star particles rather than the reference particle itself. By closely looking at the increment of leg lengths for 3 leg star particles infused in SRD fluid we found that for smallest leg length $\mathrm{L}=0.0$ the particles are dispersed almost homogeneously inside the simulation box. As the leg length increases the peak is starting to show up separately and becomes much visible from $\mathrm{L}=1.5$ and at $\mathrm{L}=3.5$ the highest peak is found at the very beginning. After the leg length increases more than the there are two distinguished peak shows up that contains a huge drop of $g(r)$ near the mid of maximum radial distance. This drop is also minimized at the largest leg length $\mathrm{L}=6.0$ where the peak shows first and then the peaks dropped gradually because of the homogeneity of particles dispersion. Again for 5 leg star particles there is also zero $g(r)$ value at the initial radial distances. The highest peak is founded right after that although the latter peaks are very closer to each other. As with the increase of leg lengths the first peak is distinguished more clearly where the latter peaks goes down and for $\mathrm{L}=3.0$ we found a sharp first peak that is gone down rapidly after that and reaches to value close to 1 . Finally for 7 leg star particles similar zero g(r) value at initial radial distance having peaks almost equal to each other at $\mathrm{L}=0.0$. But with increment of leg length the highest peak is occurred at the very beginning that goes sown sharply and maintain few equal height peaks close to 1 . 


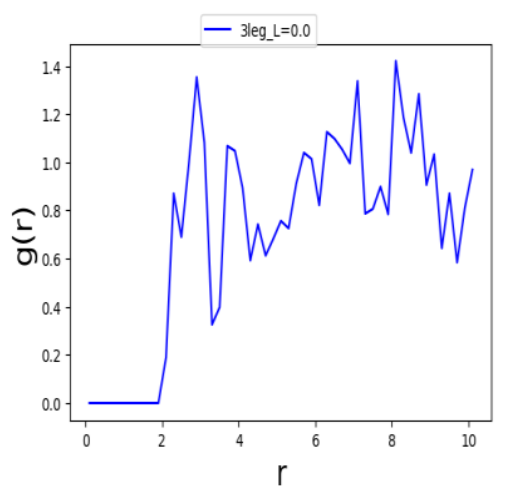

(a) 3 leg L $0.0 ; \varphi=0.18$

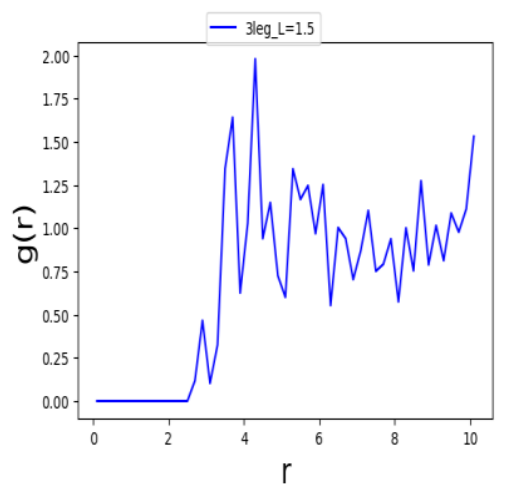

(d) 3 leg L1.5; $\varphi=0.34$

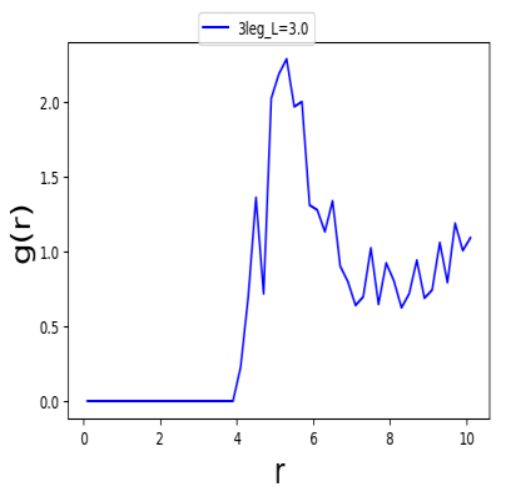

(g) 3 leg L3.0; $\varphi=0.54$

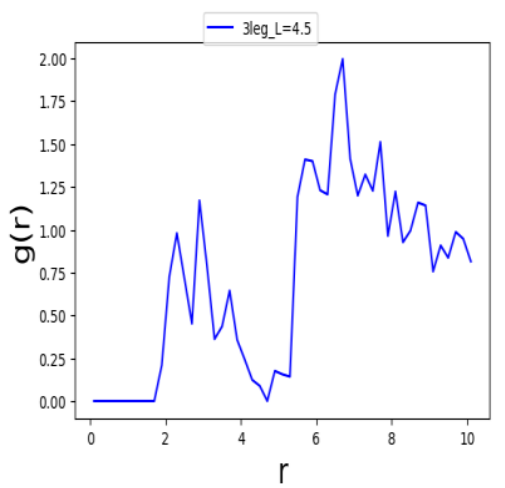

(j) 3 leg L4.5; $\varphi=0.74$

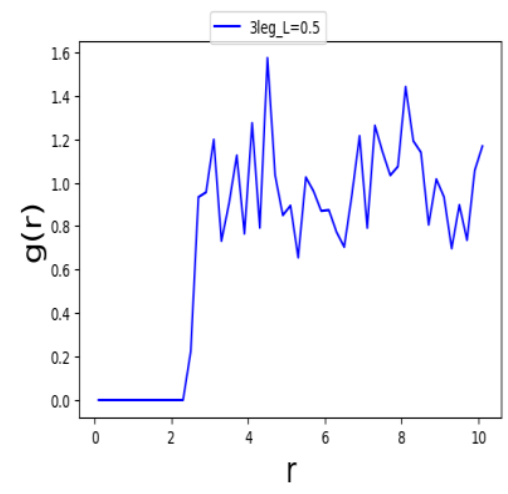

(b) 3leg L0.5; $\varphi=0.23$

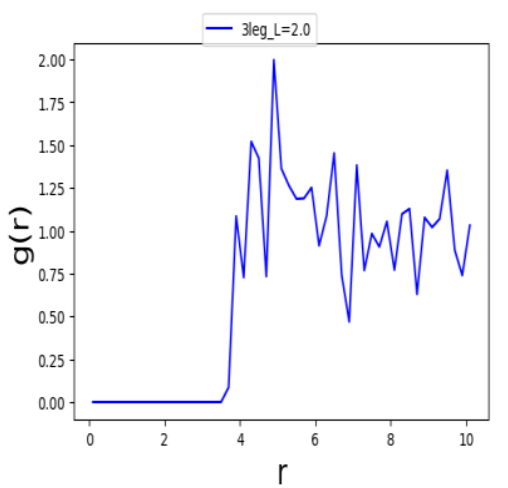

(e) 3 leg L2.0; $\varphi=0.44$

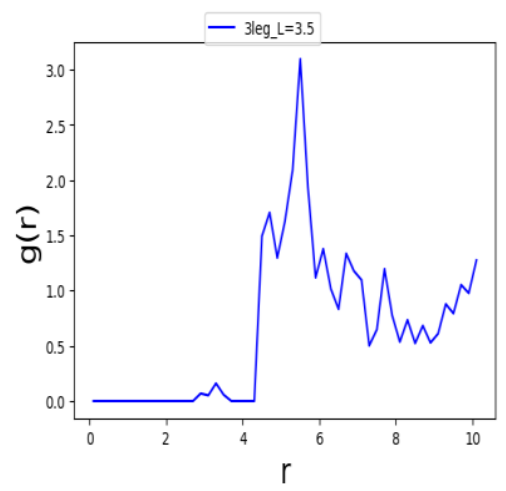

(h) 3 leg L3.5; $\varphi=0.59$

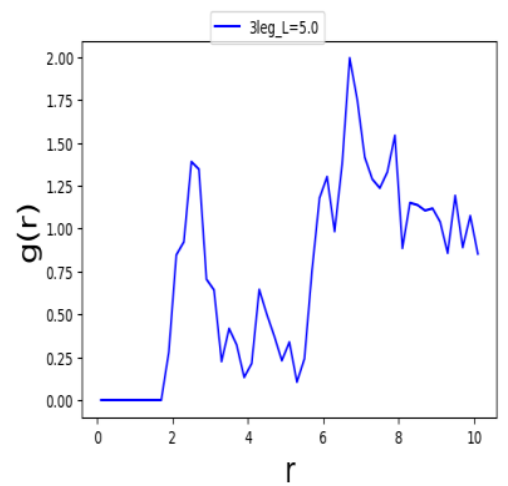

(k) 3 leg L5.0; $\varphi=0.79$

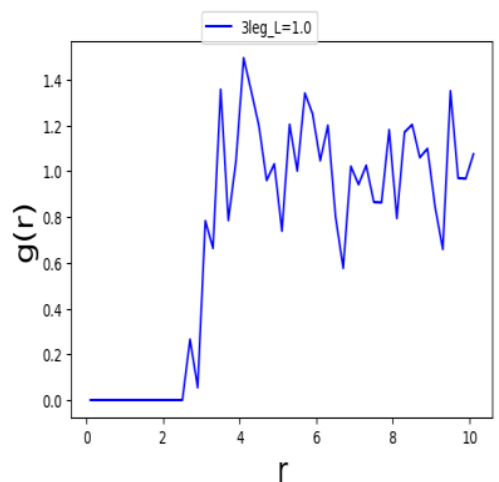

(c) 3 leg L1.0; $\varphi=0.28$

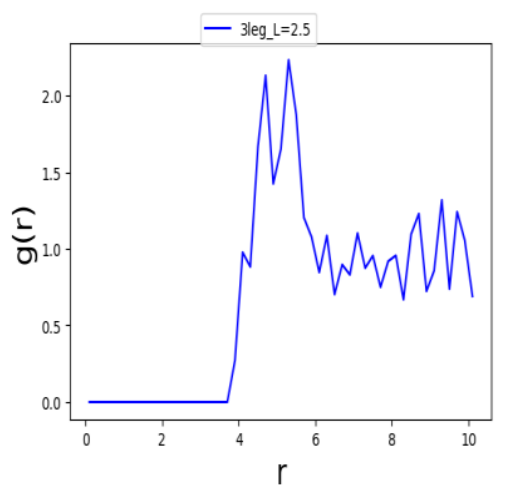

(f) 3 leg L2.5; $\varphi=0.49$

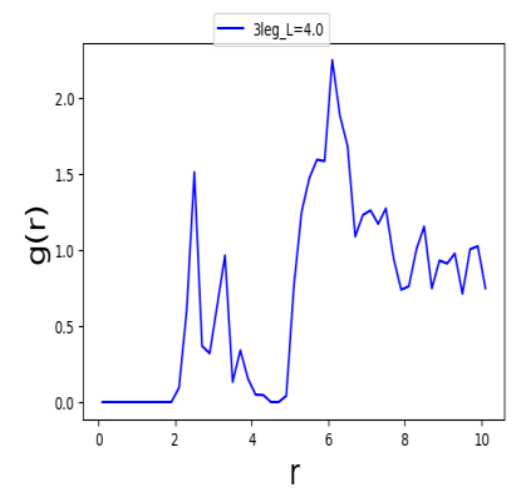

(i) 3 leg L4.0; $\varphi=0.69$

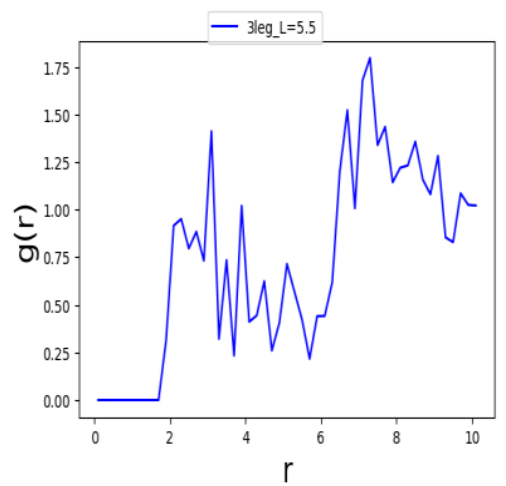

(1) 3 leg L5.5; $\varphi=0.84$ 


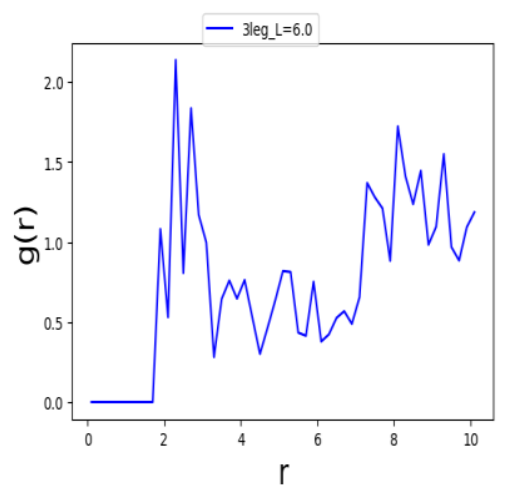

(m) 3 leg L6.0; $\varphi=0.94$

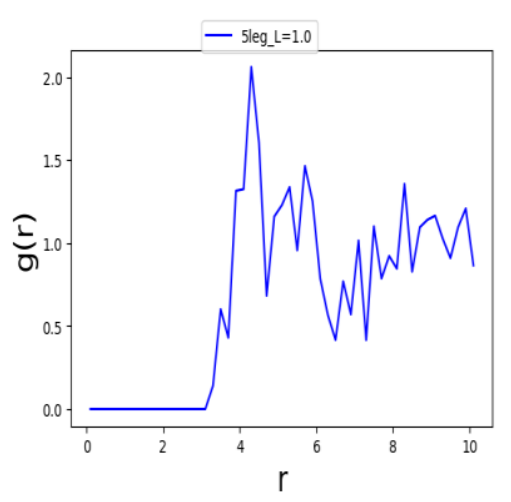

(p) $5 \operatorname{leg} \mathrm{L} 1.0 ; \varphi=0.40$

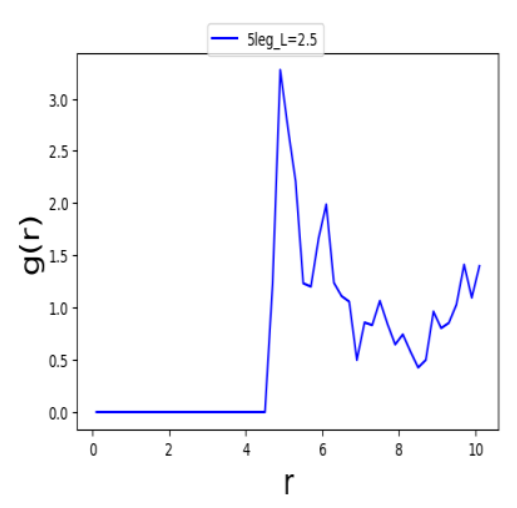

(s) 5 leg L2.5; $\varphi=0.72$

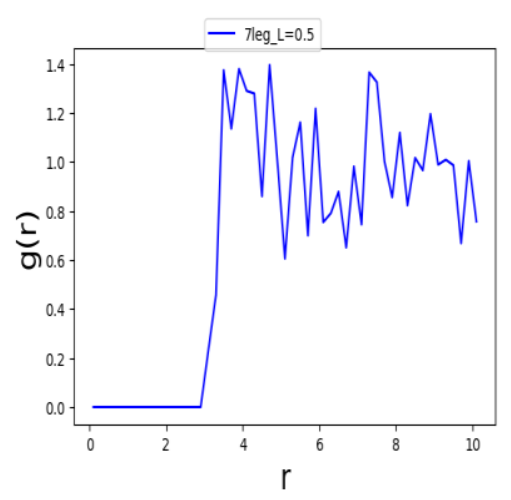

(v) 7leg L0.5; $\varphi=0.37$

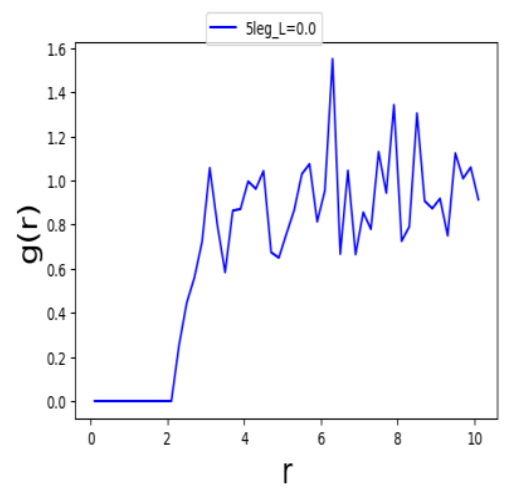

(n) $5 \operatorname{leg} \mathrm{L} 0.0 ; \varphi=0.22$

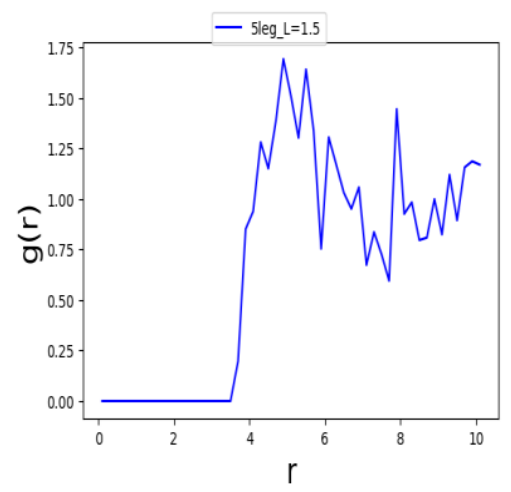

(q) 5 leg L1.5; $\varphi=0.47$

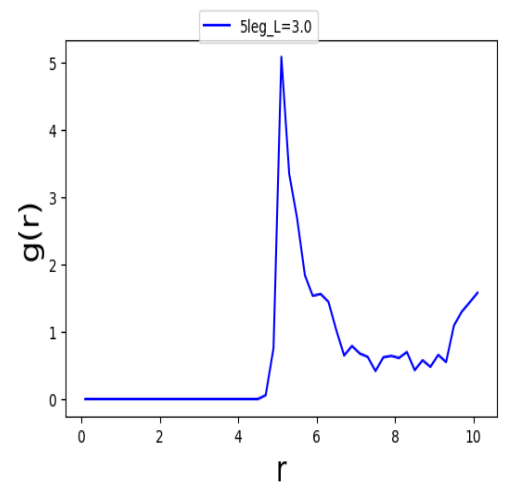

(t) 5 leg L $3.0 ; \varphi=0.80$

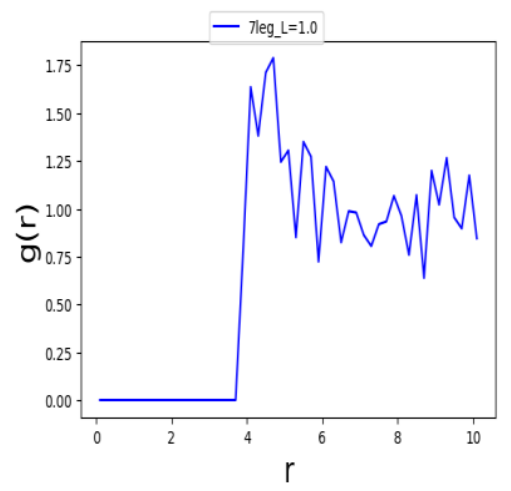

(w) 7leg L1.0; $\varphi=0.50$

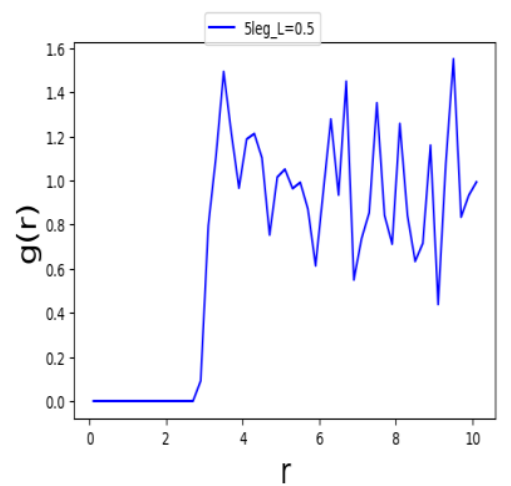

(o) 5 leg L $0.5 ; \varphi=0.30$

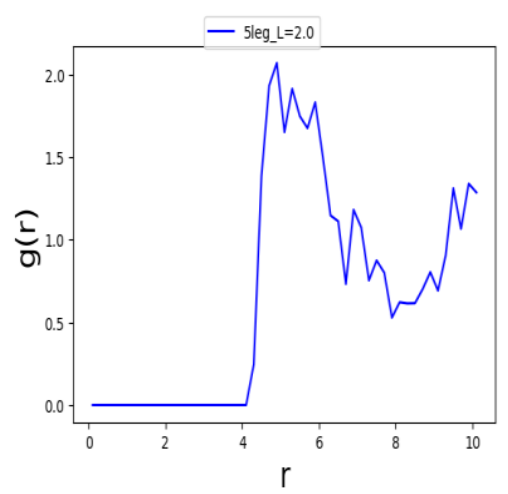

(r) 5 leg L $2.0 ; \varphi=0.62$

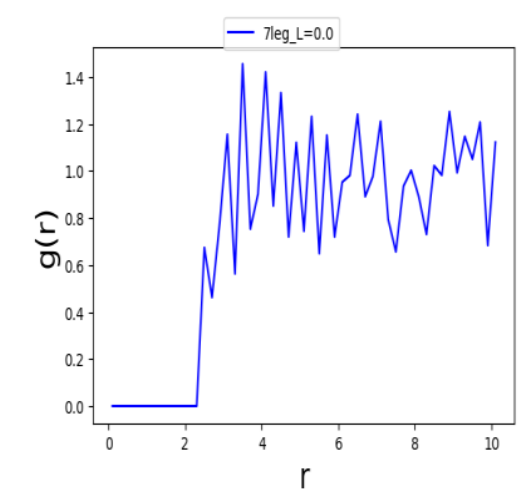

(u) 7leg L0.0; $\varphi=0.25$

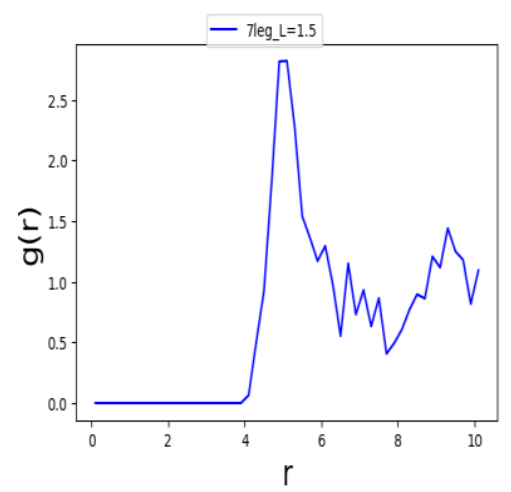

(x) 7 leg L1.5; $\varphi=0.60$ 


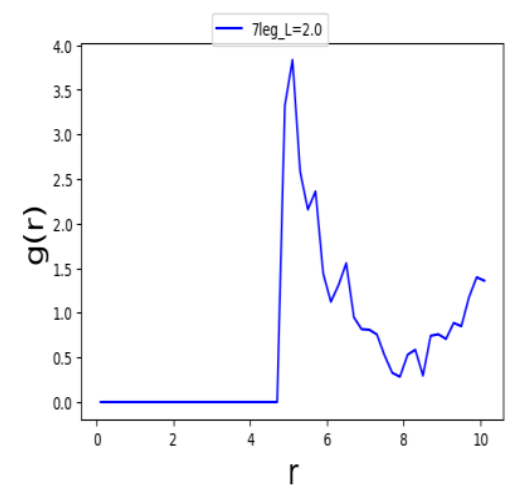

(y) 7 leg L $2.0 ; \varphi=0.84$

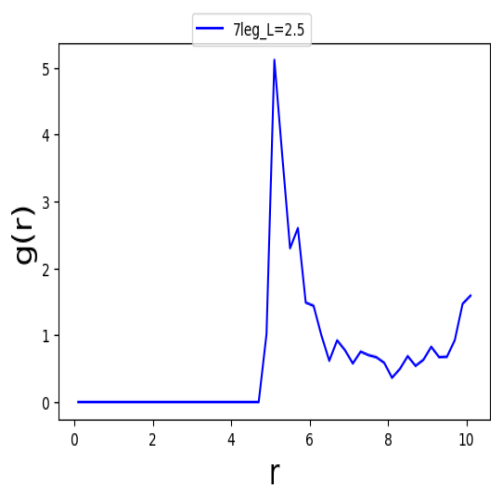

(z) 7leg L2.5; $\varphi=0.96$

Figure 4.12: Radial distribution function plot with respect to gradual increment of leg length for (a)-(m) 3 leg star particles with packing fraction $0.18,0.23,0.28,0.34,0.44,0.49,0.54$, 0.59, 0.69, 0.74, 0.79 and 0.84, (n)-(t) 5 leg star particles with packing fraction 0.22, 0.30, 0.40, $0.47,0.62,0.72$ and $0.80(\mathrm{u})-(\mathrm{z}) 7$ leg star particles with packing fraction $0.25,0.37,0.50,0.60$, 0.84 and 0.96 .

\section{CHAPTER-5}

\section{SUMMARY AND CONCLUSIONS}

From our study of Molecular Dynamics simulation using LAMMPS for fluids with infused particles of various shapes we have calculated the fluid viscosity in a way that is different than the direct methods. By varying the star particles leg length and number of legs we created different system with different packing density and plotted the viscosity with respect to leg length and packing fraction. It gives a clear view where the viscosity starts to jump due to the jamming transition. It is pretty clear from our work that jamming transition increases the solid like property of our system which was more fluidic initially and this shifting occurs due to the rise of fluids apparent viscosity. We have plotted the shear stress vs strain rate that shows that the slope (viscosity) increases as the strain rate increase which indicates the shear thickening of fluid. We also use the Herschel-Bulkley model for fitting the stress-strain plot and the model gives the exponent values greater than 1 for almost every cases that also indicates the shear thickening behavior at higher strain rate. The direct plotting of viscosity vs strain rate also shows the same shear thickening states. Then we choose the pressure parameter to evaluate the 
jamming transition and used power law to see how the exponent and jamming packing density behaves. Those also gave us the same result as we found before. Moreover we have plotted the pressure and shear stress with respect to total strain to prove that for different configurations having different initial conditions but same leg length with same number of legs finally reaches to a steady state and loose its initial memory for sufficiently long runs. Finally we conclude our work by trying to find how the structure behaves for different systems containing star particles with variable number of legs and leg lengths by calculating diffusivity and pair correlation function. We found the diffusivity decreases gradually as the system is turning into solid like state due to jamming. The radial distribution function also gives a clear probability distribution how the stars are dispersed in these system.

We believe there are so many details remaining in this field to work in future. The system could be studied more precisely for finding the exact jamming packing fraction by considering a larger system than us and doing critical scaling analysis [86]-[88] on that. Also we have used 30 star particles for an area of 30*30 square unit. Future study could increase and decrease these particle numbers and area of simulation box. Also the number of leg and leg length could be varied differently than ours and establish a relationship between them with ours one. Finally the shape of star particles could be modified such a way so that the jamming becomes more evident with much lower packings.

\section{CHAPTER-6 REFERENCES}

[1] P. J. Pritchard and J. C. Leylegian, Fox and McDonald's Introduction to fluid mechancis, Eighth. Massachusetts, USa: john wiley \& sons, inc., 2011.

[2] S. W. Cho, H. J. Jung, and I. W. Lee, "Smart passive system based on magnetorheological damper," Smart Mater. Struct., vol. 14, no. 4, pp. 707-714, 2005.

[3] H. J. Jung, I. H. Kim, and J. H. Koo, “A multi-functional cable-damper system for vibration mitigation, tension estimation and energy harvesting," Smart Struct. Syst., vol. 7, no. 5, pp. 379-392, 2011.

[4] Y. Lee and D. Y. Jeon, "Study on the vibration attenuation of a driver seat using an MR fluid damper," J. Intell. Mater. Syst. Struct., 2002.

[5] R. Stanway, "Smart fluids: current and future developments," Mater. Sci. Technol., 
vol. 20, no. 8, pp. 931-939, 2004.

[6] M. R. Jolly, J. D. Carlson, and B. C. Muñoz, "A model of the behaviour of magnetorheological materials,” Smart Mater. Struct., vol. 5, no. 5, pp. 607-614, 1996.

[7] J. de Vicente, D. J. Klingenberg, and R. Hidalgo-Alvarez, "Magnetorheological fluids: a review,"Soft Matter, vol. 7, no. 8, p. 3701, 2011.

[8] J. Rabinow, “The Magnetic Fluid Clutch," Trans. Am. Inst. Electr. Eng., vol. 67, no. 2, pp. 1308-1315, 1948.

[9] J. D. Carlson, "What Makes a Good MR Fluid?," J. Intell. Mater. Syst. Struct., vol. 13, no. 7-8, pp. 431-435, Jul. 2002.

[10] M. Růžička, "Electrorheological Fluids: Modeling and Mathematical Theory," vol. 1748, 2000.

[11] W. M. Winslow, "Induced fibration of suspensions," J. Appl. Phys., vol. 20, no. 12, pp. 1137-1140, 1949.

[12] J. P. Coulter, K. D. Weiss, and J. D. Carlson, "Engineering applications of electrorheological materials," J. Intell. Mater. Syst. Struct., 1993.

[13] P. Berger, N. B. Adelman, K. J. Beckman, D. J. Campbell, A. B. Ellis, and G. C. Lisensky, "Preparation and Properties of an Aqueous Ferrofluid," J. Chem. Educ., vol. 76, no. 7, p. 943, 1999.

[14] "Functional Particles - Matyjaszewski Polymer Group - Carnegie Mellon University." [Online]. Available: https://www.cmu.edu/maty/materials/Properties-of-welldefined/functional-particles.html. [Accessed: 03-Jun-2018].

[15] A. J. Liu and S. R. Nagel, Eds., Jamming and Rheology. New york, USA: Taylor \& Francis, 2001.

[16] A. J. Liu and S. R. Nagel, "Jamming is not just cool any more," Nature, vol. 396, no. 6706, pp. 21-22, 1998.

[17] T. A. Marschall, S. V. Franklin, and S. Teitel, "Compression- and shear-driven jamming of U-shaped particles in two dimensions," Granul. Matter, vol. 17, no. 1, pp. 121-133, 2015.

[18] S. Papanikolaou, C. S. O'Hern, and M. D. Shattuck, "Isostaticity at frictional jamming," Phys. Rev. Lett., vol. 110, no. 19, pp. 1-5, 2013.

[19] D. Bi, J. Zhang, B. Chakraborty, and R. P. Behringer, "Jamming by shear," Nature, vol. 480, no. 7377, pp. 355-358, 2011.

[20] M. Van Hecke, "Jamming of soft particles: Geometry, mechanics, scaling and isostaticity," J. Phys. Condens. Matter, vol. 22, no. 3, 2010.

[21] S. Torquato and F. H. Stillinger, "Jammed hard-particle packings: From Kepler to Bernal and beyond," Rev. Mod. Phys., vol. 82, no. 3, pp. 2633-2672, 2010.

[22] N. D. Sims, R. Stanway, A. R. Johnson, D. J. Peel, and W. A. Bullough, "Smart fluid damping: Shaping the force/velocity response through feedback control," J. Intell. Mater. Syst. Struct., vol. 11, no. 12, pp. 945-958, 2001.

[23] J. Ouellette, "Smart Fluids Move into the Marketplace," Ind. Phys., vol. 9, no. 6, pp. 
14-17, 2004.

[24] M. . Allen and D. J. tildesley, computer simulation of liquids, 1st ed. oxford, UK: oxford university press, 1987.

[25] D. Frenkel and B. Smit, understanding molecular simulation from algorithm to applications, Second. oxford, uk: academic press, 1995.

[26] D.C.Rapaport, the art of molecular dynamics simulation, 1st ed. Cambridge, UK: Cambridge university press, 1995.

[27] J. P. Hanse and I. R. McDonald, theory of simple liquids, Third. oxford, UK: academic press, 1986.

[28] H. Goldstein, C. Poole, and john Safko, Classical mechancis, Third. addison wesly, 1950 .

[29] S. Plimpton, "Fast Parallel Algorithms for Short - Range Molecular Dynamics," J. Comput. Phys., vol. 117, no. June 1994, pp. 1-19, 1995.

[30] K. Lykov, X. Li, H. Lei, I. V. Pivkin, and G. E. Karniadakis, "Inflow/Outflow Boundary Conditions for Particle-Based Blood Flow Simulations: Application to Arterial Bifurcations and Trees," PLOS Comput. Biol., vol. 11, no. 8, p. e1004410, Aug. 2015.

[31] “LAMMPS Molecular Dynamics Simulator,” Sandia National Laboratories. [Online]. Available: http://lammps.sandia.gov/.

[32] N. Gravish and D. I. Goldman, "Entangled granular media," Fluids, Colloids Soft Mater. An Introd. to Soft Matter Phys., vol. 208001, no. May, pp. 341-354, 2018.

[33] D. R. Heine, M. K. Petersen, and G. S. Grest, "Effect of particle shape and charge on bulk rheology of nanoparticle suspensions," J. Chem. Phys., vol. 132, no. 18, pp. 1-6, 2010 .

[34] M. Z. Miskin and H. M. Jaeger, "Adapting granular materials through artificial evolution,” Nat. Mater., vol. 12, no. 4, pp. 326-331, 2013.

[35] C. S. O'Hern and M. D. Shattuck, "Granular materials: Highly evolved grains," Nat. Mater., vol. 12, no. 4, pp. 287-288, 2013.

[36] A. E. Eiben and J. E. Smith, Introduction to Evolutionary Computing. 2015.

[37] S. Torquato and Y. Jiao, "Dense packings of the Platonic and Archimedean solids," Nature, vol. 460, no. 7257, pp. 876-879, 2009.

[38] H. Krupp, "Particle adhesion theory and experiment," Adv. Colloid Interface Sci., 1967.

[39] L. Massimilla and G. Donsì, "Cohesive forces between particles of fluid-bed catalysts," Powder Technol., 1976.

[40] A. Otsuka, K. Iida, K. Danjo, and H. Sunada, "Measurements of the adhesive force between particles of powdered organic substances and a glass substrate by means of the impact separation method. 1. Effect of temperature," Chem. Pharm. Bull., 1983.

[41] D. Chandler, Introduction to Modern Statistical mechancis, First. New york, USA: oxford university press, 1987. 
[42] C. W. Gear, Numerical initial value problems in ordinary differential equations. Englewood Cliffs, NJ: Prentice_Hall, 1971.

[43] D. Potter, Computational Physics. New york, USA: John Wiley \& Sons Ltd, 1972.

[44] W. C. Swope, H. C. Andersen, P. H. Berens, and K. R. Wilson, "A computer simulation method for the calculation of equilibrium constants for the formation of physical clusters of molecules: Application to small water clusters," J. Chem. Phys., vol. 76, no. 1, pp. 637-649, 1982.

[45] S. Nosé, "Constant Temperature Molecular Dynamics Methods Limitations in simulations in the microcanonical ensemble," Prog. Theor. Phys. Suppl., no. 103, p. 46, 1991.

[46] “Distance Matrix - CS 477/577 Computer Simulation \&amp; Modeling." [Online]. Available: http://wiki.cs.umt.edu/classes/cs477/index.php/Distance_Matrix. [Accessed: 05-Jun-2018].

[47] W. Liu, B. Schmidt, G. Voss, and W. Müller-Wittig, "Accelerating molecular dynamics simulations using Graphics Processing Units with CUDA," Comput. Phys. Commun., vol. 179, no. 9, pp. 634-641, 2008.

[48] "Molecular structure; Structure, Molecular." [Online]. Available: https://lookfordiagnosis.com/mesh_info.php?term=molecular+structure\&lang=1. [Accessed: 05-Jun-2018].

[49] A. J. Liu and S. R. Nagel, "The Jamming Transition and the Marginally Jammed Solid," Annu. Rev. Condens. Matter Phys., vol. 1, no. 1, pp. 347-369, 2010.

[50] M. E. Cates, J. P. Wittmer, J. P. Bouchaud, and P. Claudin, "Jamming, force chains, and fragile matter," Phys. Rev. Lett., vol. 81, no. 9, pp. 1841-1844, 1998.

[51] "pair style soft command - LAMMPS documentation.” [Online]. Available: http://lammps.sandia.gov/doc/pair_soft.html. [Accessed: 06-Jun-2018].

[52] Z. Zhang and S. C. Glotzer, "Self-Assembly of Patchy Particles," Nano Lett., vol. 4, no. 8, pp. 1407-1413, Aug. 2004.

[53] W. Humphrey, A. Dalke, and K. Schulten, "VMD: Visual molecular dynamics," Journal of Molecular Graphics, vol. 14, no. 1. pp. 33-38, 1996.

[54] J. Kingsley and E. Tüzel, “A PARTICLE-BASED METHOD FOR THE SIMULATION OF COMPLEX FLUIDS AND POLYMER SOLUTIONS Major Qualifying Project Report completed in partial fulfillment of the Bachelor of Science degree at Worcester Polytechnic Institute,” 2011.

[55] M. Hecht, J. Harting, M. Bier, J. Reinshagen, and H. J. Herrmann, "Shear viscosity of claylike colloids in computer simulations and experiments," Phys. Rev. E - Stat. Nonlinear, Soft Matter Phys., vol. 74, no. 2, pp. 1-12, 2006.

[56] M. K. Petersen, J. B. Lechman, S. J. Plimpton, G. S. Grest, P. J. In 'T Veld, and P. R. Schunk, "Mesoscale hydrodynamics via stochastic rotation dynamics: Comparison with Lennard-Jones fluid," J. Chem. Phys., vol. 132, no. 17, 2010.

[57] G. Gompper, T. Ihle, D. M. Kroll, and R. G. Winkler, "Multi-particle collision dynamics: A particle-based mesoscale simulation approach to the hydrodynamics of 
complex fluids," Advances in Polymer Science, vol. 221, no. 1. pp. 1-87, 2009.

[58] M. Hecht, J. Harting, T. Ihle, and H. J. Herrmann, "Simulation of claylike colloids," Phys. Rev. E - Stat. Nonlinear, Soft Matter Phys., vol. 72, no. 1, pp. 1-16, 2005.

[59] A. Malevanets and R. Kapral, "Solute molecular dynamics in a mesoscale solvent," J. Chem. Phys., 2000.

[60] A. Lamura, G. Gompper, T. Ihle, and D. M. Kroll, "Multi-particle collision dynamics: Flow around a circular and a square cylinder," Europhys. Lett., 2001.

[61] "fix srd command - LAMMPS documentation." [Online]. Available: http://lammps.sandia.gov/doc/fix_srd.html. [Accessed: 06-Jun-2018].

[62] S. Stalter et al., "Molecular dynamics simulations in hybrid particle-continuum schemes: Pitfalls and caveats," Computer Physics Communications. 2017.

[63] A. Malevanets and R. Kapral, "Mesoscopic model for solvent dynamics," J. Chem. Phys., vol. 110, no. 17, pp. 8605-8613, 1999.

[64] D. S. Viswanath, T. K. Ghosh, D. H. L. Prasad, N. V. K. Dutt, and K. Y. Rani, "INTRODUCTION," in Viscosity of Liquids, Dordrecht: Springer Netherlands, 2007, pp. $1-8$.

[65] "Viscosity of Newtonian and Non-Newtonian Fluids." [Online]. Available: http://www.rheosense.com/applications/viscosity/newtonian-non-newtonian. [Accessed: 05-Jun-2018].

[66] "viscosity measurements." [Online]. Available: http:/www.cscscientific.com/csccientific-blog/bid/98427/How-Can-I-Measure-Viscosity. [Accessed: 05-Jun-2018].

[67] D. S. Viswanath, T. K. Ghosh, D. H. L. Prasad, N. V. K. Dutt, and K. Y. Rani, "VISCOMETERS," in Viscosity of Liquids, Dordrecht: Springer Netherlands, 2007, pp. 9-107.

[68] P. Koumoutsakos, "MULTISCALE FLOW SIMULATIONS USING PARTICLES," Annu. Rev. Fluid Mech., vol. 37, no. 1, pp. 457-487, 2005.

[69] B. D. Todd and P. J. Daivis, "Nonequilibrium molecular dynamics simulations of planar elongational flow with spatially and temporally periodic boundary conditions," Phys. Rev. Lett., vol. 81, no. 5, pp. 1118-1121, 1998.

[70] D. S. Bolintineanu, G. S. Grest, J. B. Lechman, F. Pierce, S. J. Plimpton, and P. R. Schunk, "Particle dynamics modeling methods for colloid suspensions," Comput. Part. Mech., vol. 1, no. 3, pp. 321-356, 2014.

[71] S. H. Lee and P. T. Cummings, "Shear viscosity of model mixtures by nonequilibrium molecular dynamics. I. Argon-krypton mixtures,” J. Chem. Phys., 1993.

[72] F. Müller-Plathe, "Reversing the perturbation in nonequilibrium molecular dynamics: An easy way to calculate the shear viscosity of fluids," Phys. Rev. E - Stat. Physics, Plasmas, Fluids, Relat. Interdiscip. Top., vol. 59, no. 5, pp. 4894-4898, 1999.

[73] S. R. de (Sybren R. Groot and P. (Peter) Mazur, Non-equilibrium thermodynamics. New York: Dover Publications, 1984.

[74] L. D. (Lev D. Landau and E. M. Lifshitz, Fluid mechanics, 2nd ed. 
Elsevier/Butterworth-Heinemann, 2004.

[75] J. Crank, The Mathematics of Diffusion, Second. New york, USA: oxford university press, 1956.

[76] “Democritus: The Mean Square Displacement." [Online]. Available: http://community.dur.ac.uk/mark.wilson/Democritus/Theory/msd.html. [Accessed: 06Jun-2018].

[77] “compute msd command - LAMMPS documentation." [Online]. Available: http://lammps.sandia.gov/doc/compute_msd.html. [Accessed: 06-Jun-2018].

[78] “Democritus: The Radial Distribution Function.” [Online]. Available: http://community.dur.ac.uk/mark.wilson/Democritus/Theory/rdf.html. [Accessed: 06Jun-2018].

[79] F. Saija, S. Prestipino, and P. V. Giaquinta, "Entropy, correlations, and ordering in two dimensions," J. Chem. Phys., vol. 113, no. 7, pp. 2806-2813, 2000.

[80] M. A. Rao, Rheology of Fluid, Semisolid and Solid Foods, Third. Springer US, 2014.

[81] P. Olsson and S. Teitel, "Herschel-bulkley shearing rheology near the athermal jamming transition," Phys. Rev. Lett., 2012.

[82] S. E. Stephenson, "The relationship between simple shear and pure shear for a simple fluid without coupling effects," Rheol. Acta, 1986.

[83] "Simple and Pure Strain." [Online]. Available: http://www.geosci.usyd.edu.au/users/prey/Teaching/Geol-3101/Strain/STRAINd.htm. [Accessed: 10-Jun-2018].

[84] A. Bjorck, Numerical Methods for least Squares Problems, First. Philadelphia, USA: Society for Industrial and Applied Mathematics (SIAM), 1996.

[85] C. O'hern, L. Silbert, and S. Nagel, "Jamming at zero temperature and zero applied stress: The epitome of disorder," Phys. Rev. E, vol. 68, no. 1, p. 11306, 2003.

[86] P. Olsson and S. Teitel, "Critical scaling of shearing rheology at the jamming transition of soft-core frictionless disks," Phys. Rev. E - Stat. Nonlinear, Soft Matter Phys., vol. 83, no. 3, pp. 2-5, 2011.

[87] M. Otsuki and H. Hayakawa, "Critical scaling near jamming transition for frictional granular particles," Phys. Rev. E - Stat. Nonlinear, Soft Matter Phys., vol. 83, no. 5, pp. 5-10, 2011.

[88] P. Olsson and S. Teitel, "Critical scaling of shear viscosity at the jamming transition," Phys. Rev. Lett., vol. 99, no. 17, pp. 1-4, 2007. 